\title{
Developing a Cell Based Screen for Inhibitors of Two Component Signal Transduction in Mycobacteria
}

\author{
A thesis \\ submitted in partial fulfilment \\ of the requirements \\ for the degree of \\ Master of Science in Molecular Microbiology \\ At \\ Victoria University of Wellington \\ By

\section{Sandi Grainne Dempsey}

School of Biological Sciences

Victoria University of Wellington

New Zealand

2009 


\section{ABSTRACT}

The growing number of drug resistant strains of Mycobacterium tuberculosis appearing worldwide has had an enormous impact on the ability to control and treat Tuberculosis (TB). Discovering new anti-TB drugs is of paramount importance to the global effort for TB eradication. The success of the pathogen is largely due to its inherent ability to remain in a non-replicating or latent state for extended periods of time. In order to achieve this shift it requires tightly controlled signal transduction mechanisms to respond to its host environment. Two component systems (TCS) are one example of signalling mechanisms employed by prokaryotes and are ideal candidates for antibacterial drug targets. It is understood that many TCS are conserved in a large number of organisms, they are often essential to the virulence and persistence of pathogens and they are virtually exclusive to prokaryotes. In this study three Mycobacterium smegmatis TCS were selected; DevS/DevR, MtrB/MtrA and SenX3/RegX3. Promoters under the control of these systems were cloned into an optimised mycobacterial high copy number GFP reporter plasmid and subject to a number of in vitro stress conditions to ascertain induction conditions for these systems. As expected the DevS/DevR controlled hspX promoter was responsive to oxygen starvation and the SenX3/RegX3 controlled phoA was induced by phosphate starvation. Interestingly, phoA and $m$ trA were also induced by magnesium chelator EDTA in minimal media.

The phoA and mtrA promoter constructs were then used for in vitro high throughput bioassays with a number of compound libraries in order to screen for any inhibitory activity on each of the target systems. A phosphorylation inhibitor included in one of the screens, oleic acid, indicated that this assay could potentially be used to screen 
for TCS inhibitors, but no novel compounds were found in this study. As a proof of principle, known TCS inhibitors palmitoleic and oleic acid were employed to show a dose dependent inhibition mtrA expression. This method could potentially be expanded to other TCS of Mycobacterium smegmatis and Mycobacterium bovis BCG, or other signal transduction systems such as one component regulators and serine threonine kinases. 


\section{ACKNOWLEDGEMENTS}

I would like to express my gratitude to a number of people who were central to the completion of this project; first and foremost my supervisor Dr. Ronan O'Toole, for his vision and guidance. The assistance, feedback and encouragement I have received over the last two years have been crucial to this work.

A number of other SBS staff have also given generous of their time, equipment and expertise. In particular Dr. David Bellows, who provided a number of chemical libraries and showed us how to use all the equipment necessary to apply them to our experiments. Cameron Jack, for all the technical support in so many areas from robots to plate readers to memory sticks.

A number of plasmids were acquired from other laboratories for use in this study. Helen Stutz provided the pHIGH100 and pHS201 to our facility, both of which were extremely useful for my work.

Dr. David Ackerly and all the student members of KK817 and 816 have been a fantastic group to work with. Thank you all for all the helpful advice, borrowed reagents and bad jokes. Three people in particular have been central to my work; future Doctors Jeremy Owen, Shahista Nisa and Christopher Miller. Jeremy thank your all the advice and encouragement (and for teaching me your cloning tricks!). Chris, thank you for all the technical help, from setting up library screens to fixing computer woes, as well as all the intelligent to semi intelligent conversations that we've shared over copious amounts of coffee. Sha, you've been a wonderful friend and mentor and I value all the time you took from your own work to help me with mine from the very start. 
I also wish to thank all of my flatmates, workmates, officemates and regular old matey type mates for being so supportive and understanding. In particular Katherine Troup, thank you for the constant supply of baked goods and coffee. Special thanks to Professor Barrington and David Searle for all the welcome distractions and being the greatest friends ever.

Lastly and most importantly, I wish to thank my family Dr. Susan Burgess, Dr. Graeme Dempsey, Dr. Rachel Dempsey, Rufus and Paddy who have supported me in so many ways. Mum thanks all the hours of proofreading and Rachel thanks for the mini and not so mini breaks and generally spoiling me whenever you got the chance. 


\section{CONTENTS}

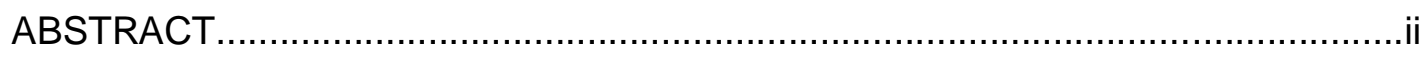

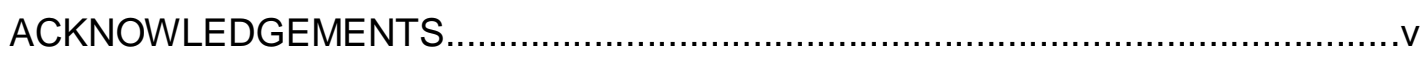

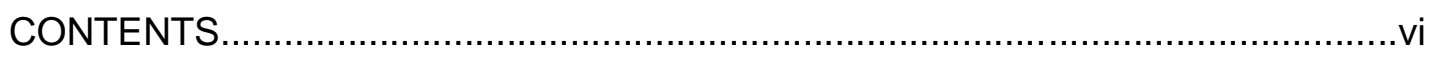

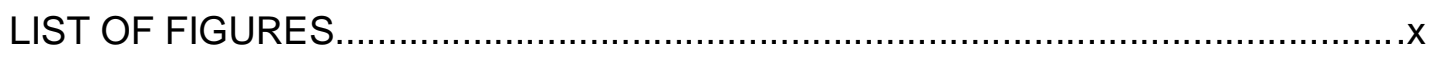

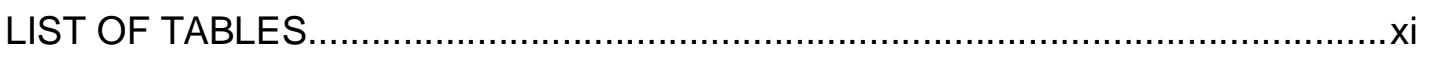

LIST OF ABBREVIATIONS...........................................................................

1. CHAPTER ONE: General Introduction...................................1

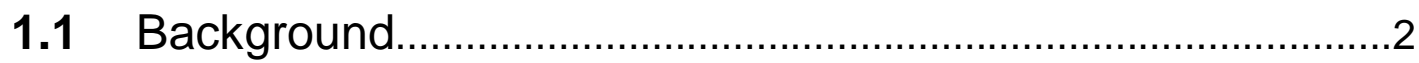

1.1.1 The Development of TB treatment................................................

1.1.2 Drugs and Resistance...........................................................

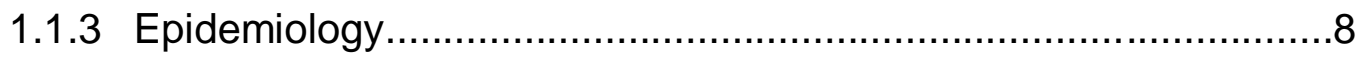

1.2 Immunology and Persistence................................................

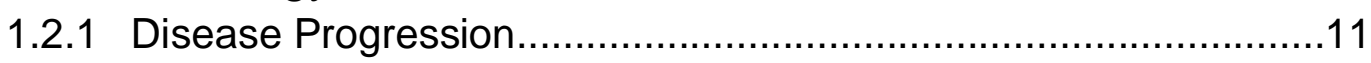

1.2.2 The Tubercular Granuloma ....................................................13

1.2.3 Endogenous Re-activation and Engogenous Re-

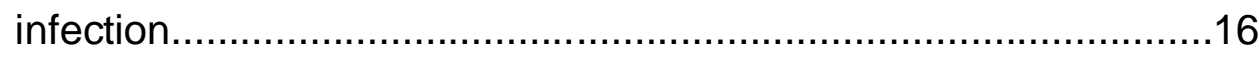

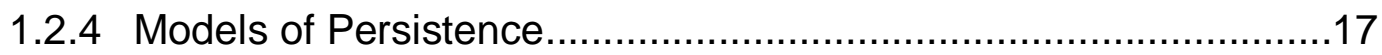

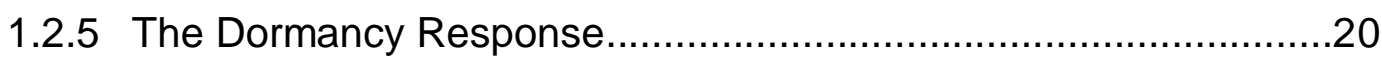

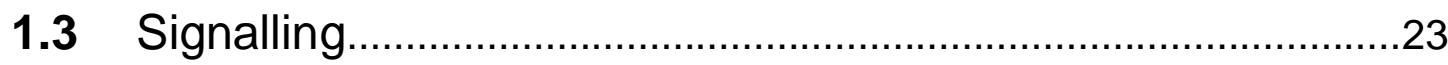

1.3.1 Classes of Signalling Molecules found in mycobacteria.................23

1.3.2 Prokaryote Two Component Signal Transduction...........................26

1.3.3 Mechanisms of Two Component Signal Transduction...................27

1.3.4 Cross talk and Networks in Two Component Systems..................29

1.3.5 The Importance of Two Component Systems in Prokaryotes.........32

1.4 Two Component Systems in mycobacteria..............................33

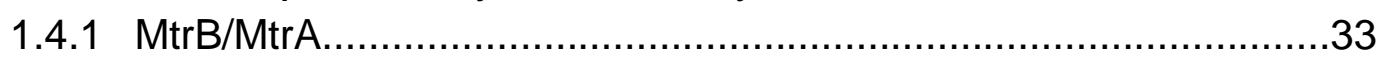

1.4.2 SenX3/RegX3

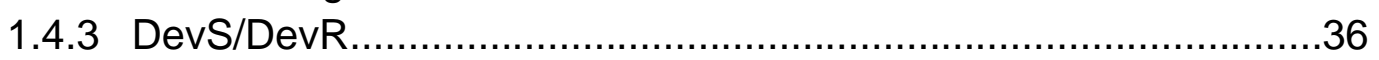

1.4.4 Two Component Systems as Drug Targets..................................38

1.5 Inhibitors of Two Component Systems ..................................40

1.5.1 Inhibition of the Histidine Kinases...............................................

1.5.2 Inhibition of the Response Regulators ......................................

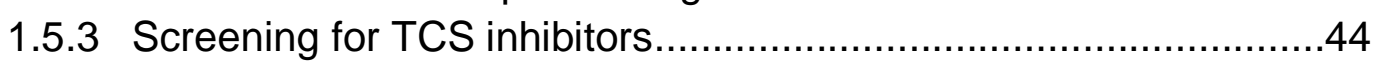




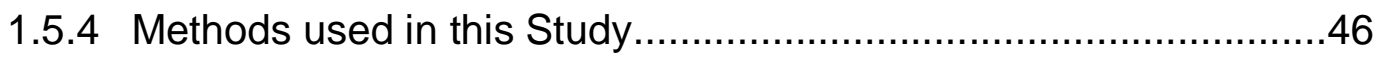

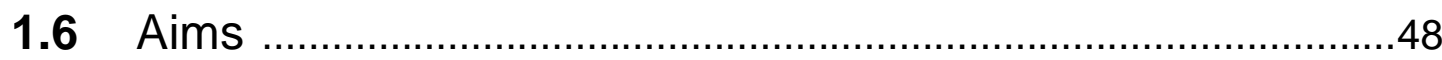

2. CHAPTER TWO: Materials and Methods ...............................49

2.1 Strains and Culture Conditions ….............................................5

2.2 Plasmids and Primers ……………….................................5

2.3 Identification of TCS dependent genes in M. tuberculosis

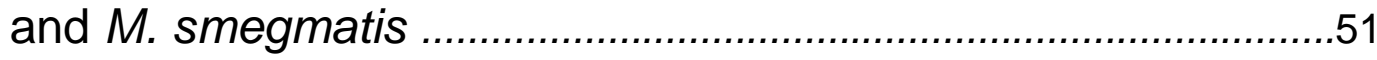

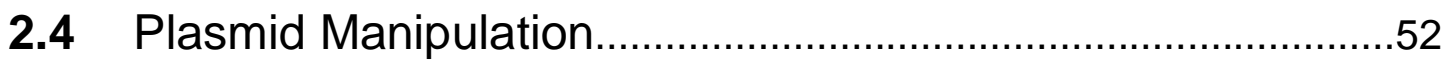

2.4.1 Amplification of Promoters from Genomic DNA ........................52

2.4.2 Ligation of promoters into Cloning Vectors and Heat Shock Transformation into E. coli......................................................53

2.4.3 Plasmid Isolation and Digestion.................................................54

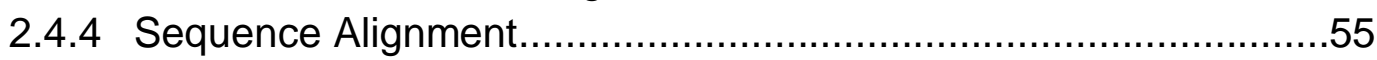

2.4.5 Low Copy Number Reporter Constructs....................................55

2.4.6 High Copy Number Reporter Constructs ....................................57

2.5 Electroporation of constructs into Mycobacterium smegmatis 60

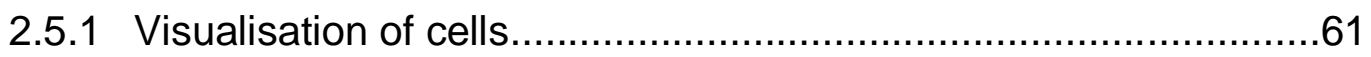

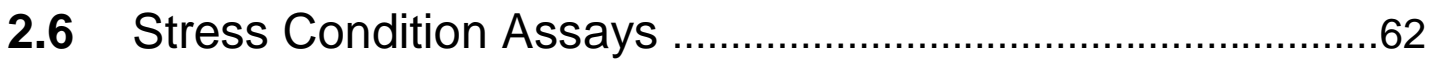

2.6.1 Oxygen Starvation Assays.......................................................62

2.6.1.1 Oxygen Starvation Assay with low copy number

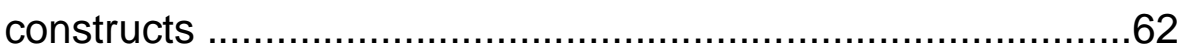

2.6.1.2 Optimised Oxygen Starvation Assay ...........................63

2.6.2 High throughput stress conditions ...........................................63

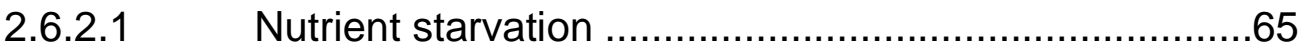

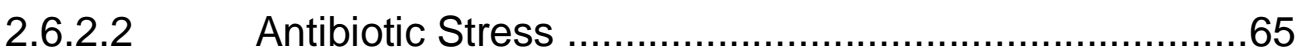

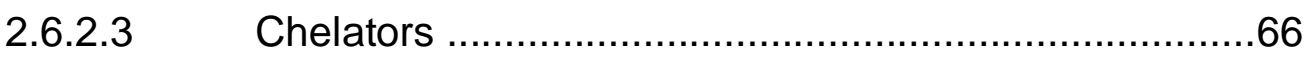

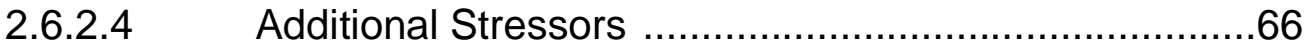

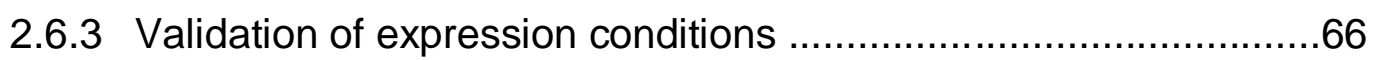

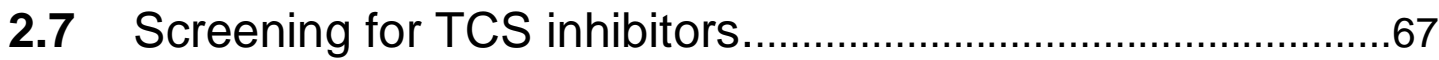

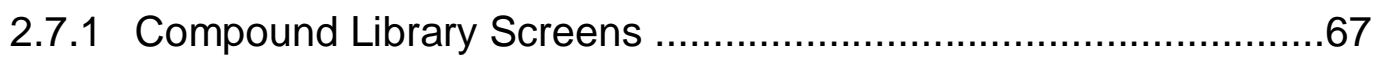

2.7.2 Palmitoleic and Oleic Acid Serial Dilution .................................68 


\section{CHAPTER THREE: Optimisation of a GFP Reporter}

Plasmid.

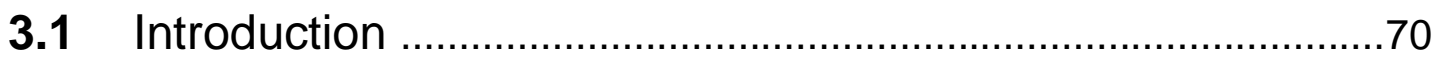

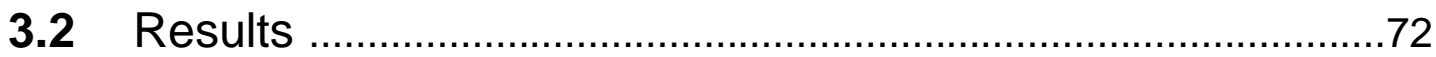

3.2.1 Amplification of GFPmut2 and oriM high by PCR ......................74

3.2.2 Switching $x y / E$ and pAL5000 with GFPmut2 and oriM high in

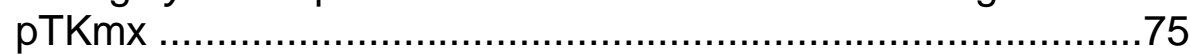

3.2.3 Comparing the fluorescence of high and low copy number plasmids in M. smegmatis .....................................................76

3.3 Inserting TCS controlled promoters into pSHigh .....................79

3.3.1 Promoter Amplification by PCR from genomic DNA ......................82

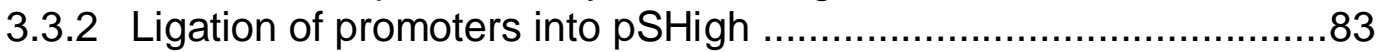

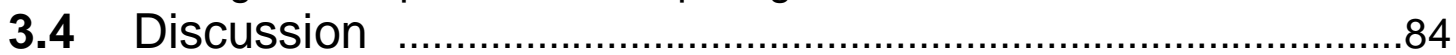

3.4.1 Optimisation of the reporter plasmid ……................................84

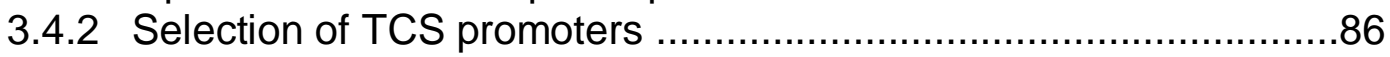

4. CHAPTER FOUR: Conditions of TCS Regulated Gene Expression

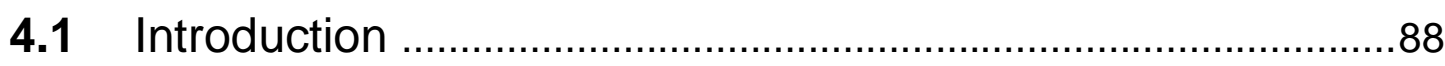

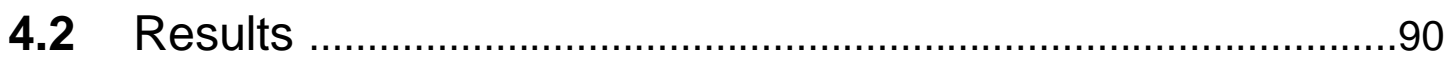

4.2.1 Expression of the DevS/DevR dependent $h s p X$ promoter .............90

4.2.2 Expression of the SenX3/RegX3 dependent phoA promoter.........93

4.2.3 Expression of the MtrB/MtrA dependent mtrA promoter ...............96

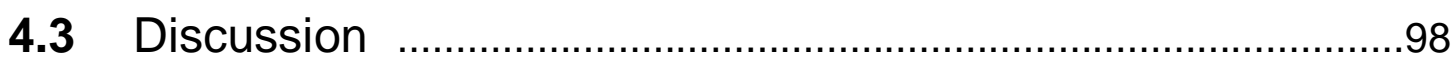

4.3.1 Expression of the DevS/DevR dependent $h s p X$ promoter .............98

4.3.2 Expression of the SenX3/RegX3 dependent phoA promoter.......100

4.3.3 Expression of the MtrB/MtrA dependent $m$ trA promoter ..............101

\section{CHAPTER FIVE: Screening Compound Libraries for TCS} Inhibitors

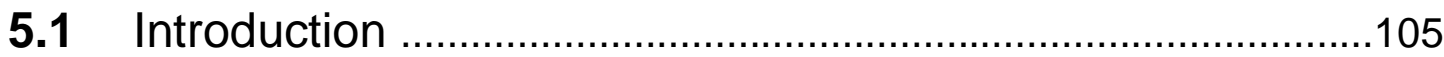

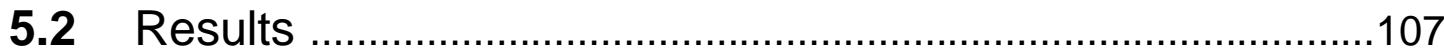

5.2.1 Drug Screens with mtra ....................................................107

5.2.1.1 Spectrum Library Screen against $m t r A$ expression ....107

5.2.1.2 LOPAC Library Screen against $m t r A$ expression .........110

5.2.1.3 NIH Library Screen against $m$ trA expression ..............113 
5.2.1.4 NIWA Collection Screen against $m$ trA expression .......116

5.2.2 Drug Screens with $p h o A$........................................................118

5.2.2.1 LOPAC Library Screen with phoA............................118

5.2.2.2 Comparison of $m$ trA and phoA LOPAC screens .........120

5.2.3 Validation of hits from Library Screens in triplicate .....................121

5.2.4 Validation of Whole Cell based screen with Oleic and Palmitoleic

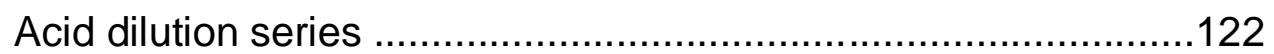

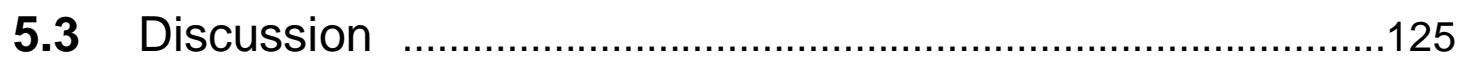

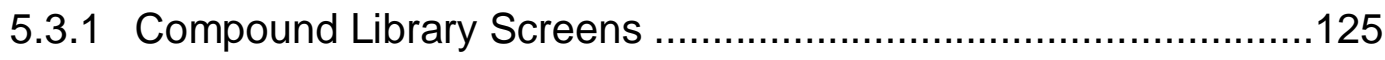

5.3.1.1 Spectrum Collection ...............................................125

5.3.1.2 NIH Collection ......................................................125

5.3.1.3 NIWA Samples .................................................126

5.3.1.4 LOPAC Library ...................................................126

5.3.2 Discussion on Screen ..............................................................127

5.3.3 Validation of the screen with cis fatty acids ..............................129

6. CHAPTER SIX: General Discussion and Future Directions..131

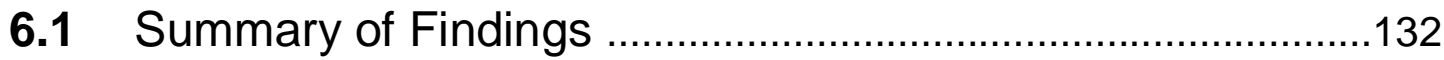

6.2 Critique of the Screen .........................................................134

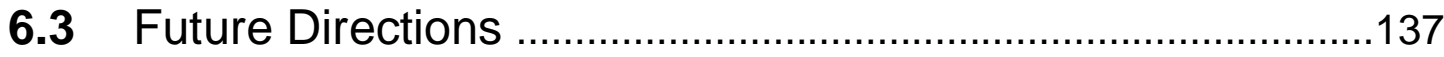

REFERENCES..................................................................140

APPENDICES …..............................................................149

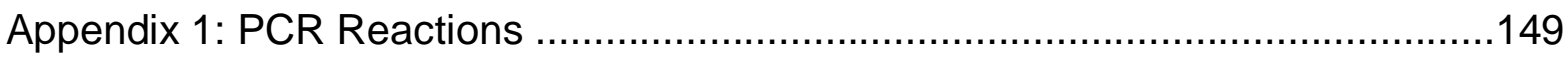

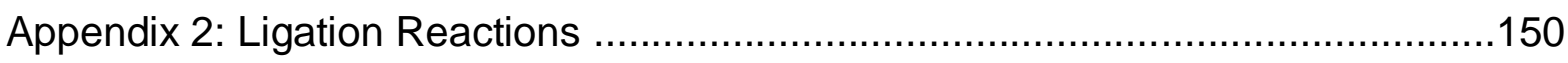

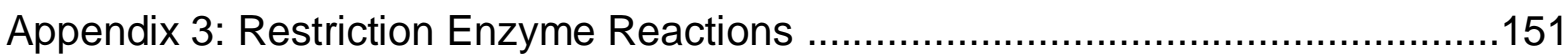

Appendix 4: Preparation of Calcium Competent DH5a cells ..............................153

Appendix 5: Preparation of Electrocompetent

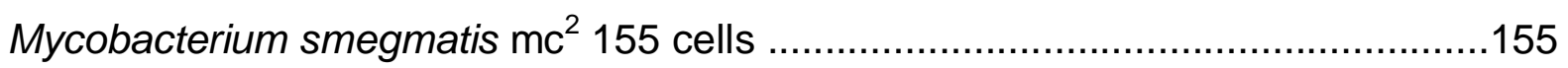

Appendix 6: Media used in this study for DH5a cells ......................................156

Appendix 7: Media used in this study for $\mathrm{mc}^{2} 155$ cells .....................................157 


\section{LIST OF FIGURES}

Figure 1.1: Disease Progression in TB 12

Figure 1.2: Two Component Signal Transduction 26

Figure 1.3: TCS inhibitors $\quad 40$

Figure 2.1: 96 well plate set up for stress condition assays $\quad 64$

Figure 2.2: 96 well plate set up for compound library screening 67

Figure 3.1: Construction of pSHigh, an optimised GFP reporter plasmid 73

Figure 3.2: Visualisation of gfpmut2 and oriM high amplified PCR products $\quad 74$

Figure 3.3: Removal of xylE and pAL5000 from pTKmx by restriction enzyme digest $\quad 75$

Figure 3.4: Visualisation of reporter constructs in M. smegmatis 77

Figure 3.5: Relative Fluorescence of Reporter plasmids in M. smegmatis $\quad 78$

Figure 3.6: Gene organisation and function of selected TCS controlled Genes

Figure 3.7: Promoters amplified from M. smegmatis and M. tuberculosis gDNA 82

Figure 3.8: Kpnl cut and uncut pSHigh plasmid

Figure 4.1: HspX expression during oxygen starvation using low copy number plasmids

Figure 4.2: HspX expression using an optimised oxygen starvation method and high copy number plasmids

Figure 4.3: The effect of phosphate starvation on phoA expression

Figure 4.4: The effect of magnesium starvation on phoA expression 95

Figure 4.5: The expression of mtrA in rich media 96

Figure 4.6: The effect of magnesium starvation on mtrA expression 97

Figure 5.1: Spectrum Screen against mtrA expression 108

Figure 5.2: LOPAC Screen against mtrA expression 111

Figure 5.3: NIH Screen against mtrA expression $\quad 114$

Figure 5.4: NIWA Screen against mtrA expression 117

Figure 5.5: LOPAC Screen against phoA expression 120

Figure 5.6: Identification of mtrA hits on LOPAC Screen against phoA 123

Figure 5.7: Oleic Acid dilution series against mtrA expression 124

Figure 5.8: Palmitoleic Acid dilution series against mtrA expression 124 


\section{LIST OF TABLES}

Table 1.1: Virulence and survival associated TCS in prokaryotes 31

Table 1.2: HKs and RRs of M. tuberculosis 33

Table 2.1: Plasmids used in this study 50

Table 2.2: Primers used in this study

Table 2.3: Stress Conditions $\quad 64$

Table 3.1: Normalised Fluorescence of High and Low Copy number

$\begin{array}{ll}\text { Plasmids } & 79\end{array}$

Table 3.2: Two Component System Controlled Genes 80

Table 5.1: Spectrum Library Candidates $\quad 109$

Table 5.2: LOPAC Library Candidates 112

Table 5.3: NIH Library Candidates 115

Table A.1 (a-c): PCR Reactions 149

Table A.2 (a-c): Ligation reactions 151

Table A.3 (a-c): Restriction Enzyme Digestion 153

Table A.4 (a-c): Alterations to HdeB media for nutrient starvation 160 


\section{LIST OF ABBREVIATIONS}

96 wp 96 well plate

ADP Adenosine diphosphate

AFB Acid Fast Bacilli

AIP Auto Inducing Peptide

Amp Ampicillin

AMP-PNP Adenosine Monophosphate p-Nitrophenol

AraLam Arabino Lipoarabinomannan

ATP Adenosine triphosphate

ATPase Adenosine triphosphatase

AWC Alan Wilson Centre

BCG Bacille Calmette-Guérin

cAMP cyclic Adenosine Monophosphate

c-di-GMP cyclic diGuanosine Monophosphate

CIP Calf Intestinal Phosphatase

CR Complement Receptor

DOTS Directly Observed Therapy

DST Drug Susceptibility Testing

DTP Developmental Therapeutics Programme

EDDA Ethylene Diamine-N,N'-Diacetic Acid

EDTA Ethylene Diamine Tetraacetic Acid

EEA-1 Early Endosome Autoantigen 1

eGFP enhanced GFP

EGTA Ethylene Glycol Tetraacetic Acid

ESR Institute of Environmental Science and Research

F Fluorescence

FACS Fluorescence Activated Cell Sorting

FITC Fluoescein Isothiocyante 
gDNA genomic DNA

GFP Green Fluorescent Protein

HdeB Hartman deBonts

HK Histidine Kinase

HPt Histidine containing Phosphotransfer domain

HTH Helix Turn Helix

HTS High Throughput Screen

IFNy Interferon gamma

INH Isoniazid

iNOS inducible Nitric Oxide Synthase

IPTG Isopropyl- $\beta$-d-thiogalactopyranoside

kan kanamycin

$\operatorname{kan}^{r}$ kanamycin resistance

LA Luria Agar

LB Luria Broth

LOPAC Library of Pharmacologically Active Compounds

LTBI Latent Tuberculosis Infection

ManLam Mannosylated lipoarabinomannan

MAP Mitogen Activated Protein

MDR Multi Drug Resistant

MIC Minimum Inhibitory Concentration

NEB New England Biolabs

NIH National Institute of Health

NIWA National Institute of Water and Atmospheric Research

OD Optical Density

oriE Origin of replication (E. coli)

oriM mycobacterial Origin of replication

oriM high mycobacterial Origin of replication (high copy number)

PCR Polymerase Chain Reaction 
PIT Phosphate Intake Transporter

PST Phosphate Specific Transporter

PZA Pyrazinamide

RBS Ribosomal Binding Site

RD-1Region of Difference

rF relative Fluorescence

RIF Rifampin

RR Response Regulator

SDW Standard Distilled Water

SM Streptomycin

STPK Serine Threonine Protein Kinase

TB Tuberculosis

TCS Two Component System

TCSi Two Component System Inhibitor

TEP TriEthylPhosphate

TLR Toll Like Receptor

TNFa Tumour Necrosis Factor alpha

WHO World Health Organisation

XDR Extensively Drug Resistant

X-gal X-galactosidase 


\section{CHAPTER ONE: \\ General Introduction}




\section{$1.1 \quad$ Background}

Mycobacterium tuberculosis has been a significant human pathogen for thousands of years ${ }^{1,2}$. Without a natural reservoir of its own, some consider it to have co-evolved with humans ${ }^{2-4}$. Studies have shown evidence of $M$. tuberculosis infection in Egyptian and Incan mummified remains up to 9,000 years ago ${ }^{2-4}$ and the domestication of cattle around 10,000 years ago ${ }^{5,6}$ was thought to be implicated in the origins of this disease ${ }^{7}$. Modern molecular methods have since indicated that the causative agent of bovine tuberculosis, Mycobacterium bovis, is a more recent species, having evolved from human specific $M$. tuberculosis ancestry ${ }^{3,8}$. Ancient $M$. tuberculosis DNA, containing none of the four splogiotypic groups used to distinguish the species from M. bovis, has been found in humans and animals dating back long before cattle domestication ${ }^{9}$. A recent paper suggests that modern $M$. tuberculosis may have evolved from a similar prehistoric pathogen found in Homo erectus up to 500,000 years ago ${ }^{10}$. While $M$. tuberculosis is clearly an ancient species, direct evidence of outbreaks of phthisis, consumption and the white plague cannot be traced back as far as the organism itself ${ }^{3}$.

Whether pathogenically inert or otherwise, M. tuberculosis has infected mankind for a substantial proportion of our existence. Robert Koch, who first isolated the acid fast bacillus (AFB) in 1881, estimated at the time that one in seven humans died of Tuberculosis (TB) ${ }^{11}$. Later in 1898, Smith identified different types of mycobacteria that infect livestock, who shared the hardy mycolic acid cell wall ${ }^{12}$. Since then, our knowledge of the Mycobacterium genus has expanded to 71 species, 32 of which are pathogenic, these have been classified in a Runyan scheme by various features including pigmentation and rate of growth ${ }^{12}$. More recently, the evolutionary 
distance between species has been further defined by the analysis of mycolic acid structures, genetic markers such as the 16S rRNA gene and, in the last decade, the comparison of entire genomes ${ }^{8-10}$. Members of the Mycobacterium tuberculosis complex are generally responsible for disease in humans and cattle; however a number of opportunistic Mycobacteria can give rise to infection in an immunocompromised host. Slow growers such as Mycobacterium celatum and Mycobacterium abscessus can infect patients with Acquired Immune Deficiency Syndrome (AIDS) or chronic immune suppression, while post-traumatic infection is generally caused by fast growers such as Mycobacterium goodie ${ }^{12}$. Comparing pathogenic and non-pathogenic species has been a useful tool in understanding the evolution of the Mycobacterium tuberculosis complex and its host-specific virulence 13

Mycobacterium smegmatis, a non pathogenic soil bacterium, has been extensively used as a model organism owing to its rapid doubling time of three hours compared

with $M$. tuberculosis' 12 hours ${ }^{12,14}$. Another model organism is the vaccine strain $M$. bovis BCG (Bacille Calmette-Guérin) whose sequence is $99.95 \%$ identical to that of M. tuberculosis ${ }^{15}$. These and other models will be discussed in section 1.2.3.

\subsubsection{The Development of TB Treatment}

TB has been a human disease for thousands of years and a range of treatments and therapies have been explored. Many significant events have lead to the current understanding and treatment of TB and other infectious diseases today. The Royal College of Physicians was set up in 1518 by Henry VIII to fight the bubonic plague and meant that physicians of the time could monitor infectious outbreaks in order to 
circumvent the devastating effects disease was having on a rapidly growing population ${ }^{16}$. The burden of TB was probably intensified by overcrowding and urbanisation, becoming a major cause of death by the Industrial Revolution ${ }^{17}$. TB was no exception to the emerging idea that living conditions play as significant a role as the disease itself, something which is still a very important factor in TB control ${ }^{16}$. Records show deaths attributed to TB in England and Wales fell from 62,633 to 27,871 between 1878 and 1940, testament to the effect of an improved standard of living on the disease ${ }^{16}$.

Another common feature of infectious endemic is war ${ }^{18}$. Devastating outbreaks of infectious diseases in post war Europe fast tracked the antibiotic era with the discovery of penicillin in 1928 by Fleming and then streptomycin (SM) in 1944 by Waksman, Schatz and Bugie ${ }^{16-18}$. Prior to this point, the most common form of treatment for TB had been fresh air and bed rest ${ }^{19}$ so Waksman's discovery in 1943 of a broad spectrum antibiotic that eliminated M. tuberculosis infection in most patients revolutionised its treatment and lead to a sharp decrease in mortality.

Preventative therapy by immunisation with a live attenuated strain of $M$. bovis BCG was pioneered in 1921 and first given to "at risk" groups such as nurses and medical students in $1949^{16}$. In one of the first large-scale medical research trials in the United Kingdom, 52, 239 children were immunised between 1950 and 1952 with the attenuated strain of $M$. bovis BCG ${ }^{16}$. The incidence of TB decreased $79 \%$ between 1954 and 1965 which was probably a combination of drug and vaccine efficacy as well as an improved standard of living, which reduced the infection rate ${ }^{16}$. 
Thousands were saved by vaccination and antibiotic development, but it did not take long for SM resistant strains of $M$. tuberculosis to emerge ${ }^{20}$. The discovery of pyrazinamide (PZA), isoniazid (INH) and rifampin (RIF) meant that TB could be relatively well controlled and combined therapy could be administered to avoid the emergence of resistant strains ${ }^{21,22}$. Eventually a growing number of patients presented strains resistant to each of the available anti-tubercular agents prompting scientists in the field to search for new classes of antibiotics.

Presently, drug resistant strains are classified as either multi drug resistant (MDR) or extensively drug resistant (XDR) based on the number and class of antibiotics to which susceptibility has been lost. MDR strains of $M$. tuberculosis are defined by the World Health Organisation (WHO) as having "resistance to at least INH and rifamycin" and XDR is described as "resistance to at least INH and RIF among firstline anti-TB drugs, resistance to any fluoroquinolone, and resistance to at least one second-line injectable aminoglycoside (amikacin, capreomycin, or kanamycin)" ${ }^{23}$. M. tuberculosis' ability to acquire genotypic and phenotypic resistance to these drugs is of great importance to physicians administering these drugs and to drug discovery efforts ${ }^{23}$.

\subsubsection{Drugs and resistance}

The treatment of TB has evolved from concoctions of distilled milk infused with earth worms and snails to modern synthetic pro drugs like $\mathrm{INH}^{20,24}$. It is impossible to say how effectively some of the early remedies such as cod liver oil, arsenic and iodine worked to reduce the TB burden at the time, but some interesting discoveries began to emerge at the turn of the $20^{\text {th }}$ century. In 1912 studies employed the use of 
cultured Bacillus subtilis and Bacillus mesentericus to inhibit the growth of $M$. tuberculosis in vitro and in a guinea pig model ${ }^{20}$. Later, the use of sulfonamides showed great anti-mycobacterial potential in vitro, but were far too toxic for use in the treatment of pulmonary $\mathrm{TB}^{20}$.

Streptomycin (SM) was isolated from Streptomyces griseus in 1943 and before the end of 1944 it was being used in clinical trials against TB, showing efficacy in vivo and in vitro ${ }^{20,25}$. SM was quickly moved to industrial scale production as it showed an effect on a broad spectrum of organisms including Gram negative, Gram positive and acid fast bacteria ${ }^{25}$. SM is an aminoglycoside antibiotic which acts on bacterial ribosomes inhibiting translation initiation and causing misreading ${ }^{26}$. Various strains have been sequenced to identify polymorphisms responsible for drug resistance in mycobacteria recorded by the drug resistance mutation database (http://www.tbdreamdb.com) ${ }^{27}$. Genes encoding the ribosomal subunits $r r s$ and $r p s L$ have been implicated in resistance to SM and other aminoglycoside ${ }^{22,26}$.

Current first line drugs include $\mathrm{INH}$, rifampin, ethambutol (ETB) and pyrazinamide. INH acts on mycolic acid synthesis, inhibiting cell wall synthesis ${ }^{28}$. It is a nicotinamide-derived drug first discovered in the 1950s, and proved more effective than any anti TB drug of its time ${ }^{24}$. Resistant isolates were discovered the same year as the drug. However, it was nearly half a century before genetic analysis of these isolates lead to the elucidation its mode of action ${ }^{24}$. INH is a pro drug activated by the M. tuberculosis KatG protein and certain polymorphisms in this gene give rise to $\mathrm{INH}$ resistance ${ }^{24}$. PZA is an interesting drug, owing to its inactivity in vitro $^{29}$. The mode of action of PZA is currently unknown, but its effect in an anaerobic environment is far more pronounced than in aerobic conditions ${ }^{29}$. Its 
activity on so called "semi dormant" bacilli has reduced the treatment time of TB from 12 to 6 months ${ }^{29}$. A number of mutations to pncA gene and promoter region result in reduced susceptibility to this drug ${ }^{27}$.

The evolution of drug resistant strains of mycobacteria is influenced by many factors. On a clinical level, the existence of many sub populations within a host, sometimes in more than one location therein, as well as an inherent ability to survive in either an active or dormant state engenders resistance ${ }^{22}$. Host specific factors also contribute to the likelihood of drug resistance developing such as the strength of innate immunity, HIV status and whether or not a patient has been treated for TB previously ${ }^{13}$. Studies have shown that the likelihood of MDR or XDR-TB arising is much higher if the patient has been previously treated for TB or is HIV positive ${ }^{23,30}$. The latest WHO figures show that countries where the incidence of MDR and XDR-TB is highest include countries such as South Africa, Ukraine and Latvia where the number of people with HIV and AIDS is also high ${ }^{23,31}$. Previous treatment is another important factor, as low quality drugs, incorrect prescription and poor adherence will affect the probability of resistance arising ${ }^{27,32}$.

On a molecular level, mutations causing polymorphisms within a population can lead to a subpopulation of resistant bacteria ${ }^{33}$. Mutation can arise spontaneously or as a result of exogenous agents causing DNA replication errors. Point mutations, insertions, duplications or loss of genetic material can result in a change of phenotype allowing an organism to become resistant to a drug which usually knocks out an essential process ${ }^{34}$. In some ways the more critical the target to cellular replication, the more likely resistance will arise. The rate of mutation in pathogenic 
mycobacteria is relatively low compared with faster growing pathogens, however there are many genes which display variable mutation rates ${ }^{35}$.

There are many reasons $M$. tuberculosis is able to develop resistance to drugs including its extended incubation and latency period as well as the fact that most drugs act on targets essential for growth and division ${ }^{13,22}$. As well as these documented genotypic changes, the idea of reversible phenotypic drug resistance is a concept that may well be central to $M$. tuberculosis drug susceptibility. The idea that sub populations of bacteria can survive exposure to drugs by a phenotypic switch to a reductive metabolism, rather than a permanent genotypic change is a relevant argument for the case of mycobacteria ${ }^{36}$.

\subsubsection{Epidemiology}

The WHO annual surveillance report on TB for 2009 gives a summary of the current state of TB as well as strategies to combat the disease worldwide ${ }^{23}$. Encouragingly the report indicates that the number of new TB cases per capita has decreased since 2003, however with 9.27 million new cases and 1.3 million deaths in 2007 , these alarming statistics show there is still a long way to go before TB is completely eradicated ${ }^{23}$. The highest incidence per capita is in Africa with an average rate of 363 per 100,000 and 14.4 deaths in $2007^{23}$. The USA and Monaco have the world's lowest prevalence rates of 3.1 and 1.9 per 100000 respectively, while Swaziland has the highest rate with 1,226 per $100000^{23}$.

The WHO gives the latest prevalence of TB in New Zealand as 7.3 per 100,000 for 2007 and ESR (Institute of Environmental Science and Research) recorded this rate as 7.2 per 100,000 in $2008^{23,30}$. Five new cases of TB were recorded in its latest 
public health survey report for July and September 2008 all of which were in Wellington ${ }^{30}$. TB has a very strong association with poverty and age in $\mathrm{NZ}{ }^{37}$. Unlike countries with a high prevalence of HIV, the majority of TB cases are found in older people, children and people in a low income bracket ${ }^{37}$. Although globally the rate of TB has dramatically decreased in the last hundred years, thanks to the availability of antibiotics and a higher standard of living in most countries, the emergence of MDR and XDR strains of TB is of huge concern ${ }^{23}$. First line TB drugs can be used in most cases to treat TB. Second line drugs are used in cases of drug resistance; however they are more expensive, must be administered for longer and display severe side effects $^{23,37}$.

Drug resistant TB is not geographically isolated, according to the WHO 2008 report MDR TB was detected all over the world and at least one case of XDR TB was reported in all 45 countries surveyed ${ }^{23}$. Globally, it is estimated that 3.9 to $6.6 \%$ of all cases of TB are MDR ${ }^{23}$. The highest rates of MDR-TB in the world are recorded in Eastern Europe especially in Latvia and Ukraine (up to $36 \%$ of all cases), much higher than in Africa ${ }^{23}$. Data from these countries has shown that the percentage of MDR-TB is significantly different in new cases at $2.9 \%$ compared with previously treated cases at $15.3 \%{ }^{23}$. Some associate this with a sharp increase in the number of HIV cases reported in this area ${ }^{23}$, it may also be that Eastern Europeans countries have a very effective surveillance strategy for MDR and XDR-TB and are picking up more cases than other countries.

The STOP TB strategy set up in 2006 includes an expansion of the directly observed therapy (DOTS) regime to 184 countries and addresses the challenge of TB HIV co infection $^{32}$. 
In addition to an increased susceptibility to TB, a positive HIV status is significant because it will affect drug resistance and the host immune response to TB, precipitating the onset of disease ${ }^{23}$. Testing for HIV status among TB cases in high risk areas has increased from $8 \%$ to $35 \%$ since $2002{ }^{23}$. A very important part of TB research is recording information from around the world showing trends of drug resistance. Drug susceptibility testing (DST) is one way that MDR and XDR can be monitored and managed ${ }^{32}$. Although mutations causing resistance to current TB drugs are unavoidable, ensuring that the right combinations of drugs are given to patients is one way that the rate of MDR and XDR-TB can be reduced.

Currently, the discovery of new anti-TB drugs is of paramount importance in the effort to stop TB. In order to combat emerging drug resistant strains new targets need to be elucidated. Our focus is on latent TB and the following section will describe the progression, persistence and virulence of TB and why targeting latent, in addition to active, TB could help to alleviate this global epidemic. 


\section{$1.2 \quad$ Immunology and persistence}

One of the most challenging features of TB treatment is the organism's ability to maintain a dormant state within its host for long periods of time ${ }^{34,38}$. This section will outline the progression of TB with an emphasis on factors which affect the disease outcome for patients and morphological and genetic changes within $M$. tuberculosis which allow it to persist for so long.

\subsubsection{Disease progression}

M. tuberculosis is an obligate pathogen, unlike some other mycobacterial species it has no environmental niche but can live in humans asymptomatically for long periods of time ${ }^{39}$. Given that one in three humans harbours latent TB, there is in fact a huge reservoir of $M$. tuberculosis worldwide (http://www.who.int/mediacentre/factsheets/fs104/en/). Infection begins with inhalation of aerosols containing M. tuberculosis AFB to pulmonary alveoli ${ }^{36,37}$. The presence of foreign pathogenic bacteria is very quickly recognised by the host immune cells via bacterial toll like receptors (TLR) and mannose receptors ${ }^{36,38}$. Macrophages and dendrites are recruited to the site of infection where they phagocytose bacterial cells. In non-pathogenic mycobacteria this results in efficient control of the infection since macrophages induce the elimination of bacilli by phagolysosome fusion, complement activation and lymphocyte mediated killing ${ }^{40}$. During M. tuberculosis infection, most of the AFB are cleared in this manner but in approximately one third of cases, the pathogen persists by interfering with macrophage maturation ${ }^{38,41}$ as shown in figure 1.1. 


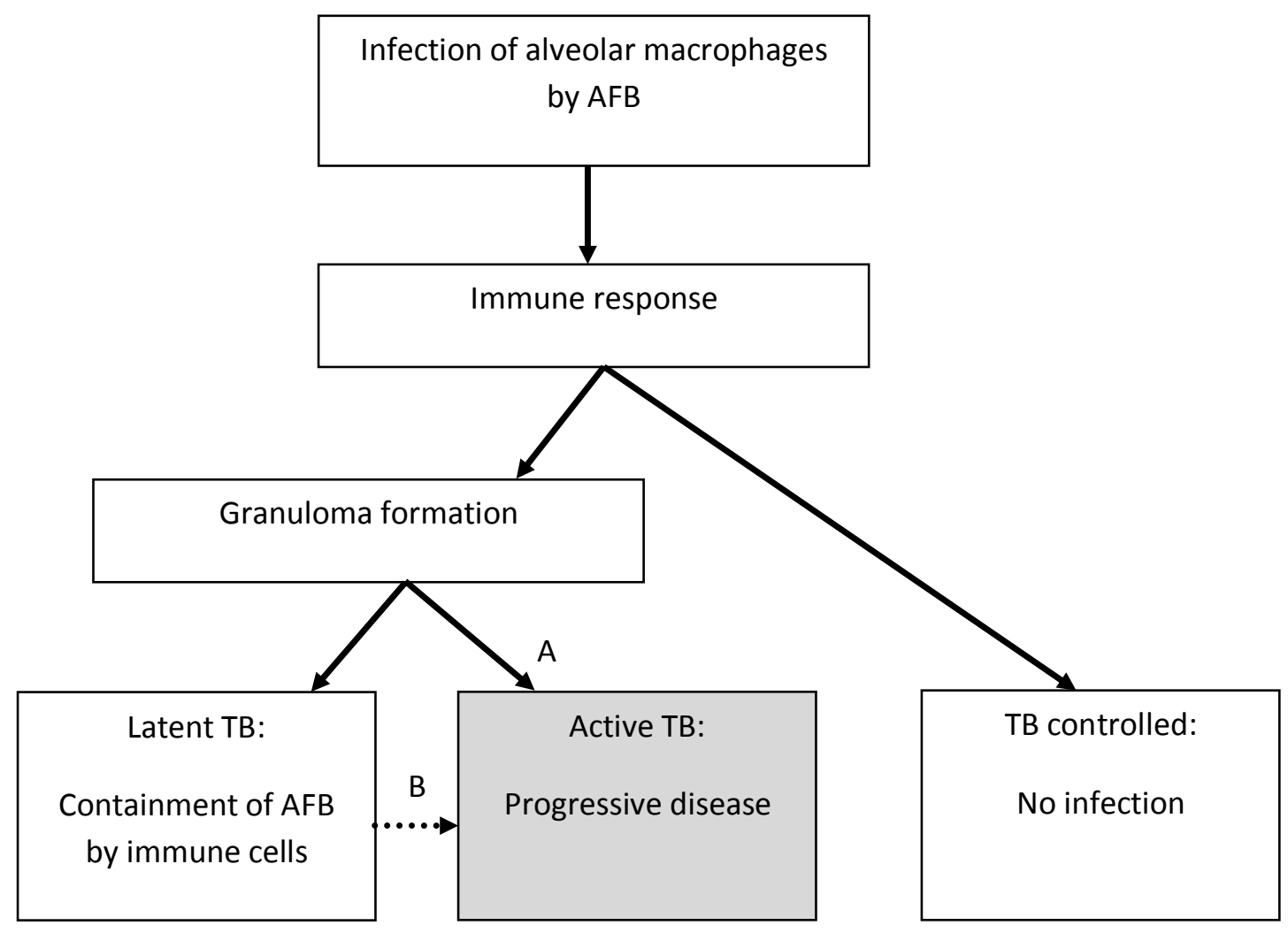

Figure 1.1: Disease Progression in TB. In most cases AFB are cleared by the immune system. Active TB results from expansion of the primary granuloma $(A)$ or re-activation $(B)$ of contained $A F B$

Various mechanisms of immune evasion have been proposed which are described in a number of reviews ${ }^{36,38-41}$. Although the infected macrophage cannot induce the release of toxic lysosomal products to the AFB containing phagosome, there is still a large immune response from the host ${ }^{40}$. This leads to a gathering of immune cells forming a tubercular lesion known as a granuloma. These characteristic lesions are a hallmark in TB diagnosis and range in size and cellular makeup depending on stage of disease ${ }^{42}$. In about $90 \%$ of individuals, the AFB are contained in the granuloma which consists of dead and foamy macrophages surrounded by activated macrophages and lymphocytes ${ }^{41,43}$. There is often a surrounding fibrotic layer leaving the AFB in a contained environment ${ }^{43,44}$. In a small number of individuals 
the initial infection will lead to primary tuberculosis after an incubation period ranging from 4 to 12 weeks ${ }^{43}$. Dissemination of the primary granuloma results in active TB by allowing AFB to spread to other regions of lung and eventually other organs of the host $^{36,37}$.

\subsubsection{The Tubercular Granuloma}

The host adaptive immune response results in the formation of a granuloma;

providing protection from but not sterilization of an $M$. tuberculosis infection ${ }^{40}$. This is also seen in other pathogens such as those of the Schistosoma and Brucella genera ${ }^{44}$.The large number of immune cells recruited to the site of infection is exaggerated during a TB infection, effectively encapsulating the AFB to the initial infection foci. After AFB enter a macrophage they are transported across the epithelial lining where they recruit more immune cells to the site. This recruitment requires the Region of Difference (RD1) virulence gene locus ${ }^{45}$. Ulrichs and others showed that the type of cell arriving at the granuloma changes with time, after 4 weeks $45 \%$ of the cells are macrophages whereas at 6 weeks $80 \%$ are lymphatic cells ${ }^{42}$.

A number of reviews describe the structure of the granuloma and the general consensus is that there is a necrotic core surrounded by concentric rings of immune cells $^{36,41,42}$. AFB reside in macrophages surrounded by a mass of cellular material consisting of dead macrophages and Langhans giant cells, the latter having formed by the fusion of epitheloid macrophages ${ }^{42}$. This layer is surrounded by a number of activated macrophages, surrounded by naive macrophages and T lymphocytes. A number of others components are involved in this organised structure including $B$ lymphocytes, dendritic cells, neutrophils, fibroblasts and extracellular matrix ${ }^{44}$. 
Surrounding tissue keeps the entire structure in check by secreting chemokines and cytokines ${ }^{42}$. The aggregation of $T$ cells around the fibrotic layer acts as mini lymph nodes surrounding the healing granuloma ${ }^{42,43}$. The conditions within the tubercular granuloma have been studied extensively using a number of models; certain areas of the granuloma have been shown to be hypoxic, nutrient and iron deprived, and contain toxic nitric oxide products ${ }^{42,44}$.

The cellular composition of the granuloma is altered depending on whether the lesion is healing or disseminated and the centre of the granuloma can either be casious or calcified $^{42}$. Other differences in these states are the presence of blood supply in an active granuloma and fibrotic coating surrounding the healing granuloma keeping AFB contained to the site of infection ${ }^{42}$. One of the most important factors affecting the rate of healing is the bacillary load present in the lungs ${ }^{45}$. Other host factors will also determine how quickly the granuloma heals, in particular the health of the individual's immune system.

There is debate as to whether the granuloma is in a constant or dynamic state ${ }^{42,45}$. The historical view of granuloma formation implies that after infection granulomas heal over time unless certain host factors trigger re-activation ${ }^{42}$. This static view implies that the dormant bacilli are contained in the granuloma, employing a reductive metabolism in order to survive in the hypoxic nutrient deprived lesion for extended periods of time until resuscitated. Numerous studies in different models have shown that TB is able to switch to a dormant state during LTBI ${ }^{45-48}$. There is, however, mounting evidence that this may not be the case for every single AFB. For example, the finding of $M$. tuberculosis DNA outside the granulomatous lesions of patients with LTBI indicates that AFB could be escaping from granulomas even 
during an $\mathrm{LTBI}^{49}$. The problem here is that the presence of DNA does not necessarily prove that live $M$. tuberculosis survives in the lung. To date the presence of RNA, indicative of live M. tuberculosis, has only been found within macrophages in non-necrotic zones of the granuloma ${ }^{44}$.

The dynamic hypothesis describes a state seen in the $M$. marinum model where the AFB are contained by fibrotic tissue surrounding the granuloma but can escape to other areas of the lung with the help of macrophages ${ }^{45}$. In this view, infection remains controlled even though the lesions are moving around the lung; more forming as older ones heal completely ${ }^{45}$. Unfortunately, which view you take is largely dependent on what model organism and host you are studying. As there is really no perfect model for LTBI in humans, all of the evidence obtained using different model organisms and hosts needs to be carefully considered. In any case, there is something unique about the ability of $M$. tuberculosis to maintain a slow growing persistent state in response to the harsh environmental conditions it faces. The granuloma effectively houses the infectious agent for extended periods of time. This can lead to endogenous re-activation of the disease in a compromised host. There is much debate as to whether the majority of current TB cases are the result of exogenous re-infection of $A F B$ or endogenous re-activation from a previous infection ${ }^{41}$. Given that so many humans harbour latent TB, yet only a small proportion of those exposed to the pathogen develop a latent or active infection, one could argue that both processes are very important in post primary TB. 


\subsubsection{Endogenous Re-activation and Exogenous Re-infection}

A number of studies attest to $M$. tuberculosis' ability to survive for long periods of time in a dormant state in vivo and in vitro ${ }^{16,45-48}$. There is evidence that TB can survive in a human host for up to 33 years ${ }^{48}$. Re-activation occurs when a granuloma disseminates and infected macrophages spread to other parts of the lung 42,50 . The ratio of cases which arise from re-activation and re-infection is unknown but it is likely that a number of host factors are involved in the outcome. A Latent TB infection (LTBI) is maintained by a delicate balance between the host immune system and the population of AFB in the granuloma ${ }^{42}$. Immune suppressant drugs such as infliximab have been shown to greatly increase the rate of re-activation ${ }^{51}$. Malnutrition, old age and co-infection with HIV have all been shown to increase reactivation rates ${ }^{13,34}$. Even genetic differences within individuals of a population can influence the odds of re-activation, for example polymorphisms on chromosome regions $2 q 21-2 q 24$ and $5 p 13-5 q 22$ have been linked to susceptibility to re-activation 52.

On the other hand many of these factors could also increase the likelihood that an individual is susceptible to re-infection. Consider that a malnourished, elderly or HIV positive patient is probably much more likely to be unable to control a new infection if they live in an area with a high risk of contagion. As we learn more about the granuloma status in latent and active TB it seems likely that both re-activation and re-infection contribute to the current epidemiology of the disease. There may be an entire spectrum of disease states in these lesions. The AFB may be replicating very slowly and causing new lesions which heal without ever causing active disease until there is a break down in the immune system control causing re-activation or an 
increase in AFB number as a result of re-infection ${ }^{45}$. In either case, the fact that TB can survive in this latent phase, providing such a large reservoir of AFB developing drug tolerance and resistance, make it an extremely dangerous pathogen.

\subsubsection{Models of Persistence}

Before discussing the ability of $M$. tuberculosis to enter dormancy, it is necessary to explain some of the models used to explore the dynamics of mycobacterial gene expression and host responses to the AFB during LTBI. H37Rv, a common lab strain of M. tuberculosis, is restricted to physical containment level 3 facilities in New Zealand. This precaution and its long doubling time of 12 hours make it a difficult organism to work with, thus other species of the mycobacterium genus have been used in its place. M. smegmatis and M. bovis BCG are commonly used to explore changes involved in dormancy ${ }^{48,53}$. Other related mycobacterial model organisms include zoonotic pathogens $M$. avium and M. marinum ${ }^{12}$.

The Wayne model is an in vitro model used to explore changes in gene expression during stationary phase ${ }^{54}$. It involves a slow starvation of oxygen to a point where the optical density (OD) of the cell culture is constant but the cells remain viable ${ }^{55}$. This model was originally established in the 1970 s and the authors discovered that genes involved in the glycoxylate shunt were up-regulated during this growth phase

${ }^{56}$. Three markers of dormancy were described namely, tolerance to anaerobiosis, unique antigen production and a significant increase in the production of glycine dehydrogenase and isocitrate lyase enzymes ${ }^{56}$. These changes mean that $M$. tuberculosis can use up less energy and persist in a slow or non-replicating state. Many of these findings have been replicated by two dimensional gel and microarray 
analysis showing upregulation of $i c /$ and ace $A$ genes during stationary phase and during in vivo infection ${ }^{57}$. Other variations on this model achieve stationary phase persistence by altering temperature, $\mathrm{pH}$, nutrient sources and nitric oxide levels ${ }^{44}$.

While in vitro models cannot replicate the hostile environment of the granuloma, many mycobacterial species exhibit similar changes as a result of oxygen starvation to those seen in vivo such as antibiotic tolerance, down regulation of a number of genes involved in active metabolism and induction of a set of genes known as the dormancy regulon ${ }^{47}$. In vivo induced genes have also been categorised by infection with human macrophage cells ${ }^{44}$. Some of these can be further broken down to groups of macrophage and granuloma activated genes ${ }^{44}$. The different subsets show that there are some intrinsic host factors required to fully appreciate dormancy in human LTBI ${ }^{44}$.

The Cornell model has been used extensively to discover more about in vivo adaptation by $M$. tuberculosis. This model involves drug induced immunosupression in mice ${ }^{36,65,66}$. After infection, a mouse will generally clear most of the AFB without causing disease until treatment with steroids causes $M$. tuberculosis re-activation. While much of what we know about in vivo gene expression and host response is derived from this model, it does have its limitations. The bacterial load required for a TB infection in mice is much higher than what is usually seen in humans ${ }^{44}$. Consequently, the mice end up with a chronic infection causing severe damage of the lung, rather than the anaerobic calcified granuloma seen in humans ${ }^{36,43}$. Another problem is that the administration of drugs is not standardised across the field which may cause discrepancies in results ${ }^{44}$. 
These problems have lead to the exploration of other host organisms. Guinea pigs are much more susceptible to $M$. tuberculosis infection and produce granulomas of similar pathology to those in humans ${ }^{36,67}$. Rabbits also show a similar pathology with caseous and calcified healing lesions however, they are resistant to $M$. tuberculosis infection and will only be infected by M.bovis ${ }^{44}$.

Using other species of mycobacteria in their specific host is another way of determining how persistence can be achieved. Although they will not fully represent the human disease, many of the mechanisms used to survive intracellular conditions are similar. Members of the $M$. avium subspecies are obligate pathogens which infect chickens and cause Crohn's disease in immune compromised humans ${ }^{12} . M$. bovis is $99.95 \%$ similar to M. tuberculosis on a nucleotide level and infects a number of animals including goats, pigs and sheep ${ }^{15}$. Interestingly $M$. bovis, which is more recently evolved than $M$. tuberculosis from the original progenitor of the complex, the loss of certain genetic elements exhibits a broader host range ${ }^{15}$.

Avirulent $M$. bovis BCG has been used as a vaccine strain for many years and a particular region that was lost in BCG is the previously mentioned RD1 region ${ }^{36,68}$. This genetic region was recently studied in $M$. marinum, which can cause disease in frogs and fish ${ }^{45}$. It is a particularly useful model as it infects macrophages and gives a similar granuloma structure to the closely related $M$. tuberculosis. The aforementioned work used this species in zebrafish embryos to determine formation and stability of granulomas in this species. 


\subsubsection{The Dormancy response}

Using the models described, significant advances have been made in elucidating the dormancy response during persistence in a granuloma. Transposon mutagenesis studies have implicated a number of genes for survival of $M$. tuberculosis at various stages of infection ${ }^{46}$. The pathogen must respond rapidly to environmental cues in order to alter its proteome and adapt to these harsh conditions using tightly regulated signal transduction mechanisms. Some of the important cellular changes will be described.

\section{The RD1 Region}

The development of the $M$. bovis BCG vaccine strain involved serial passage of the strain until it reached an avirulent state ${ }^{58}$. Genotyping the vaccine strain revealed the loss of two significant genetic regions, esat 6 and cfp10 collectively known as the RD1 region. A BCG:RD1 knock in strain displayed an increase in virulence that was not entirely restored with respect to the wild type strain ${ }^{58}$. RD1 mutants have a different morphology to wild type and do not form a granuloma structure. A recent study confirmed that this genetic region was vital in maintaining persistence in a granuloma using the $M$. marinum zebrafish model ${ }^{45}$.

The distinct layers of a granuloma suggest that these lesions are not a random mass of cellular material but an organised structure which forms as macrophages are activated by AFB, leading to aggregation of mononuclear phagocytes ${ }^{59}$. Davis et al. found that the M. marinum RD1 mutant was attenuated in its ability to form a granuloma. The main distinctions they found in macrophage aggregation from the RD1 mutant compared with wild type included differences in the speed and 
morphology of macrophages recruited to the infection foci. The granuloma expansion was also different; in wild type cells a continual expansion of the granuloma was observed through cycles of apoptosis and phagocytosis, while the mutant showed no chemotaxic recruitment of macrophages.

Other changes involved in the dormancy response include the induction of the so called dormancy regulon. Over 40 genes are induced by the DevS/ DevR two component system (TCS) in response to conditions of low oxygen and nitric oxide ${ }^{60-}$ 62. These include enzymes required for glycoxylate metabolism, shown in the previously mentioned Wayne model ${ }^{56}$, a number of other stress response genes are induced by this TCS, including the $h s p X$ gene ${ }^{62-64}$.

Changes to the cell wall structure of the pathogen involving the PhoP/ PhoR system are also noted during latency ${ }^{57,70-73}$. A review on the potential of cell wall components as drug targets describes multiple changes in the cell wall composition as a result of stationary phase in mycobacteria ${ }^{65}$. The author describes possible changes to the amount and conformation of mycolic acid, a change in the ratio of cross-linked peptidoglycan and a decrease in the level of arabinogalactan present during dormancy ${ }^{65}$.

All of the mechanisms which $M$. tuberculosis uses to evade immune responses and survive latently in an intracellular environment rely on adapting to life in the macrophage. The AFB is able to do this via a number of signal transduction mechanisms which tightly control gene expression in response to extra and intra cellular changes it encounters. The current understanding of changes to the mycolic acid structure of the AFB cell wall during infection raises questions about the efficacy 
of cell wall related drug targets, such as $\mathrm{INH}$, for treating latent TB ${ }^{65}$. The AFB's switch to a more reductive metabolism will also render drugs which target active replication poor candidates against latent infection. In order to eliminate the sizable reservoir of latent TB worldwide, new candidates that act on targets utilised during this dormancy phase are required. 


\subsection{Cellular Signalling}

Every living organism must have tightly controlled sensing mechanisms in order to survive in and respond to its particular niche. Both prokaryotic and eukaryotic cells employ a number of responsive systems and processes, without which they would be rendered defenceless in the face of any changes in their respective environments. Organisms adapt to life in specific conditions with relation to temperature, $\mathrm{pH}$, and the availability of nutrients and oxygen, to name a few. How well an organism adapts to a changing environment determines its survival and evolutionary fitness.

In M. tuberculosis, signalling molecules and systems allow the organism to live in the hostile environment of the human immune system induced granuloma for extended periods of time, making these structures attractive drug targets. Their relative distance, in terms of homology, from eukaryotic signalling systems means that inhibitors of prokaryote signal transduction are less likely to evoke a negative response in the human host. This section will highlight important prokaryote signalling mechanisms seen in mycobacteria, with an emphasis on the two component systems (TCS) which are the focus of this study.

\subsubsection{Classes of Prokaryote Signalling Molecules found in mycobacteria}

In response to external signals, prokaryotes employ single and multi protein phosphorelay systems to interpret and respond to external ligands. This results in control of gene expression by either transcriptional control or post translational modification of proteins. Histidine kinases (HKs) are generally part of two component system signal transduction. Other sorts of protein kinases include serine threonine 
and tyrosine kinases. The earliest evidence for reversible phosphorylation in prokaryotes was unearthed in the $1970 \mathrm{~s}^{66}$. This sort of mechanism had only been revealed in eukaryotes and the bacteriophage $\mathrm{T} 7$, so the discovery of serine threonine kinases in Salmonella typhimurium ${ }^{66}$ preceded a surge of interest in this area.

\section{One Component Regulators}

One component signalling, as the name implies, requires just one protein with sensor and effector domains ${ }^{67}$. Intracellular changes are recognised by the sensing domain which results in DNA binding of the effector, which acts as a transcriptional activator or repressor ${ }^{67}$. Some well known examples include the TetR and AraC proteins in E.coli, which respond to L-arabinonse and tetracycline respectively ${ }^{67}$. The simplest AraC type regulators do not require phosphorylation or communication between proteins ${ }^{67}$. Thousands of bacterial proteins share motifs with the AraC regulator but none have been found in eukaryotes ${ }^{67}$. An example of this sort of protein in mycbacteria is the iron dependent regulator IdeR. The protein contains an $\mathrm{N}$ terminal helix turn helix motif for DNA binding and two metal binding sites surrounded by the dimerization domain ${ }^{68}$. When bound to iron, the protein represses the $f x b A$ gene product ${ }^{79-81}$, which is an important enzyme in the synthesis of the $M$. smegmatis siderophore exochelin ${ }^{69}$, and activates iron storage gene bfr $A^{70}$. The ability to acquire and store iron is very important during intracellular infection and is noted as a virulence factor for $M$. tuberculosis ${ }^{82,83}$. The ide $R$ gene has been deemed essential ${ }^{71}$, probably because loss of this gene product results in an overload of iron accumulation which is toxic to the organism ${ }^{72}$. 


\section{Serine Threonine Kinases}

The first mycobacterial serine threonine protein kinase (STPK) was discovered in $1997^{73}$, since then 11 Mycobacterial STPKs have been identified named Pkn A to L, which are implicated in development, pathogenicity and stress responses ${ }^{74}$. STPKs are often implicated in development from one state to another such as in sporulation of $M$. xanthus ${ }^{75}$. They are also thought to interfere with Mitogen Activated Protein (MAP) kinases of epithelial cells in Listeria monocytogenes ${ }^{76}$. Mycobacteria, especially M. marinum, have a large number of STPKs; it is possible that they are complementing a relatively smaller number of TCS compared with other bacteria ${ }^{77}$. PknG and PknK are the only M. tuberculosis STPKs that do not posses transmembrane regions, all the other STPKs are grouped based on homology clusters or function.

Adenylyl cyclases and diguanylyl cyclases

Other transcriptional regulators are influenced by global regulators such as cyclic nucleotides cyclic adenosine monophosphate (cAMP) and cyclic diguanosine monophosphate (c-di-GMP). Bai et al have shown that cAMP has an important role in macrophage interaction as its concentration increases 50 fold during $M$. tuberculosis and $M$. bovis BCG infection ${ }^{78}$. At least 15 class III adenylyl cyclases exist in $M$. tuberculosis and 31 in $M$. leprae, a relatively high number compared with other bacterial genomes ${ }^{79,80}$. 


\subsubsection{Prokaryote Two Component Signal Transduction}

The concept of two component signal transduction, whereby one component recognises some external change and another component modulates an appropriate response, is now a hallmark of prokaryote signal transduction. Phosphorylation of histidine kinases was recognized in E.coli by Kundig and Roseman in 1971 who were studying mechanisms of sugar transport ${ }^{81}$. Later it was found that phosphorylation of such proteins can modulate changes in gene expression ${ }^{82}$ when coupled with a response regulator in a process outlined by figure 1.3. The term two component system is something of a misnomer, as it includes multistep kinases with intermediate proteins to regulate and insulate signals ${ }^{83}$. In E. coli there are 32 response regulators $(\mathrm{RR}), 23$ histidine kinases $(\mathrm{HKs})$ and 5 hybrid sensory kinases 83. These were identified by Mizuno et al using OmpR, NarL, NtrC and CheY systems as probes to search for similar open reading frames ${ }^{83}$. They described a number of common "signature sequences" of HKs and RRs. 


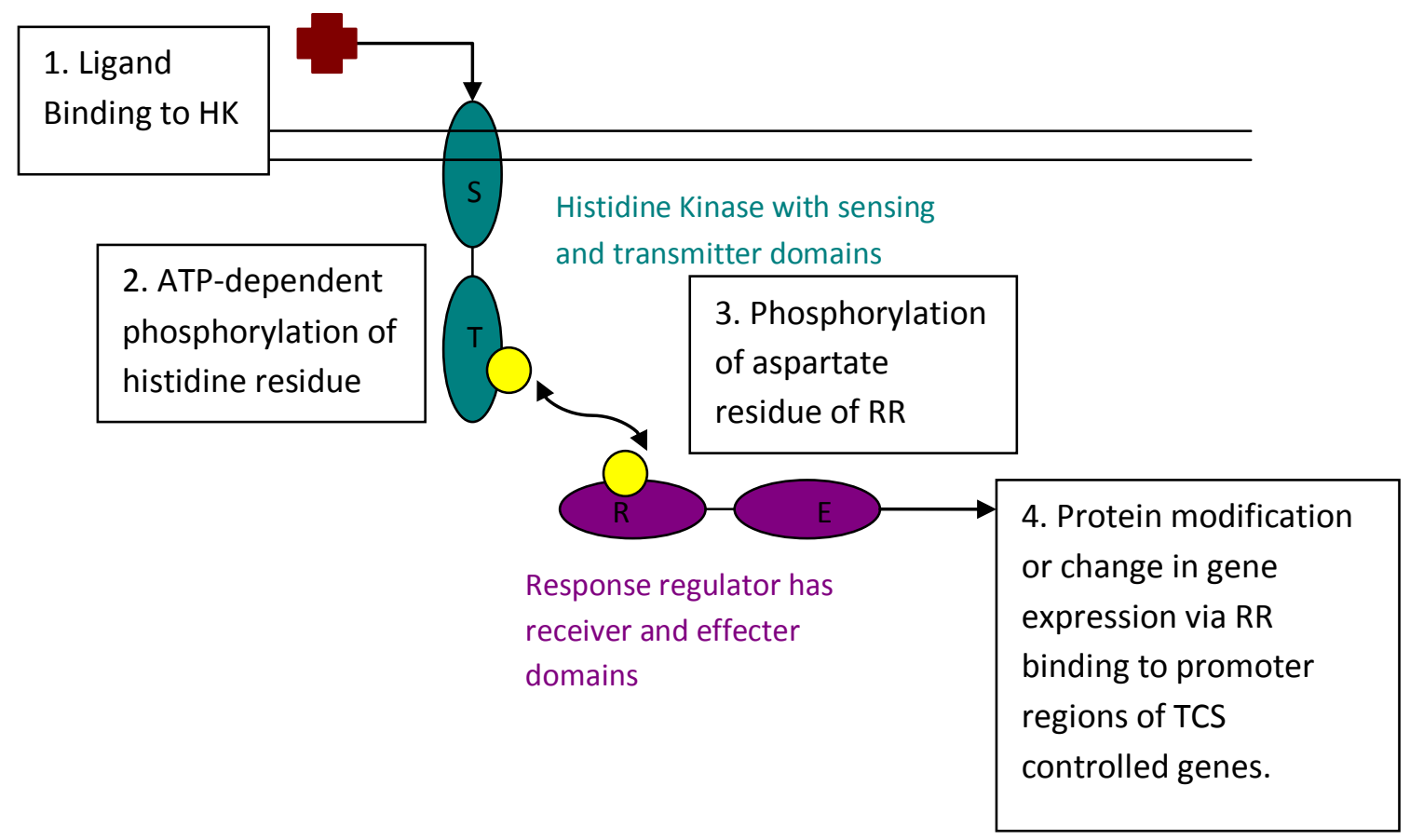

Figure 1.2: Two Component Signal Transduction. Prokaryote two component systems. Signals are relayed from membrane bound Histidine Kinase to Response Regulator via phosphorylation of conserved amino acids $\bigcirc$. Apart from ligand-specific binding domain of Histidine Kinase and effector domain of Response Regulator, many regions of these systems are well conserved among prokaryotes.

\subsubsection{Mechanisms of two component signalling}

Histidine kinases possess diverse sensing domains as well as a number of conserved transmitter domains ${ }^{84,85}$. They have been divided into one of 11 groups based on their protein sequence surrounding conserved residues on their transmitter domain namely the $\mathrm{H}$ box, $\mathrm{N}$ box, $\mathrm{D}$ box, $\mathrm{F}$ box and $\mathrm{G}$ box ${ }^{85}$. The HK usually includes one to five $\mathrm{N}$ terminal transmembrane regions responsible for ligand binding ${ }^{86}$. This part of the protein can also be periplasmic, or intracellular and connected to other transmembrane receptors ${ }^{85}$. 
As mentioned, this part of the protein is extremely variable amongst TCS. Some studies have shown the sensory domain to have a specific ligand binding preference, such as divalent cations ${ }^{87}$, others imply that certain conditions lead to a change in conformation of the protein ${ }^{88}$. In either case, this results in an ATP-dependent autophosphorylation of the $\mathrm{H}$ box of the protein. The HKs occur as dimers with catalytic domains connected by a HAMP linker motif ${ }^{84}$. The catalytic domain forms a complex with a cognate RR containing a conserved aspartate residue ${ }^{89}$. The phosphoryl group is transferred from the histidine to the aspartate residues resulting in a switch to the RR's active state ${ }^{89}$.

The RR usually contains around 120 amino acids, including the crucial aspartate and two other conserved residues ${ }^{83}$. The RR carboxy terminal effector domain is responsible for initiating the cellular responses to the ligand ${ }^{83} . \mathrm{OmpR}, \mathrm{NarL}$ and NtrC type RRs have DNA binding domains and act as transcription factors in that they directly induce or repress gene expression ${ }^{83}$. Others have an enzymatic role in the cell or directly interact with other proteins ${ }^{85}$.

There are essentially four steps common to all TCS which are illustrated in figure 1.3. An environmental stimulus is recognised, usually in the form of ligand binding. This results in a conformational change to the HK, causing autophosphorylation of the histidine residue of the HK transmitter domain by the conversion of an ATP to ADP (adenosine diphosphate). Then there is a transfer of the phosphoryl group from a histidine residue on the $\mathrm{HK}$ to an aspartate residue on a response regulator, and finally dephosphorylation of the phosphoryl group to inorganic phosphate ${ }^{89}$. 
This is a simplified model of this mechanism because there are a number of variations on this theme. Some systems involve intermediate steps for greater control and networking between signals. Multi-step phosphotransfer signalling works on the same principles as two component signalling, with an added level of complexity. HPts are histidine containing phosphotransfer domains found in some signalling systems ${ }^{84}$. This higher level of complexity is often found in eukaryote signalling systems, either to ensure that there are multiple regulatory checkpoints, to amplify a signal or so that the HPt can be an alternate target ${ }^{84}$.

\subsubsection{Cross talk and Networks in TCS Signal Transduction}

As well as having intermediate proteins, these systems can form networks to integrate signal transduction ${ }^{90}$. Pathways can be branched, in other words one HK can phosphorylate many RRs, such as is the case in the CheA/CheY/CheB system or multiple HKs can phosphorylate one $\mathrm{RR}{ }^{91}$. It is somewhat surprising, given the conserved mechanism of phosphotransfer across signalling networks, that crosstalk is not a problem ${ }^{91}$. Since there are often dozens of these systems in a cell, responding to a vast array of different signals, it is important that these phosphorelays are insulated from each other. There is evidence that, in the absence of their cognate HK, a RR can be phosphorylated by other HKS ${ }^{91}$. For example, in a PhoR deletion mutant, its native RR PhoB can be phosphorylated by another HK named VanS ${ }^{91}$. Another way to induce cross talk of signals is to over-express the HK, presumably making the protein more likely to phosphorylate another RR. This has not been shown in wild type cells, indicating that there is a high level of control over signal insulation. It is likely that RRs, which are generally more abundant in the cell than their cognate $\mathrm{HKs}$, have a preference for their own $\mathrm{HK}^{90,91}$. Also, the 
observation that HKs often negatively control RRs by de phosphorylation means that there is a smaller chance of signals going astray ${ }^{91}$.

In eukaryotic Arabidopsis thaliana, a TCS network has been analysed giving a map of hundreds of interactions between proteins involved in these systems ${ }^{92}$. A similar study in E. coli concluded that the most significant interaction between TCS was in the overlap of gene expression resulting from different systems ${ }^{93,94}$.

\subsubsection{The Importance of TCSs in Prokaryotes}

The signal relays described usually culminate in an alteration of gene expression. Large numbers of genes are either switched on or off as a result of the RR activation. Control of cell osmolarity in E. coli by EnvZ/ OmpR is a well understood example of how the cell is capable of maintaining turgor pressure ${ }^{84}$. Controlling the expression of membrane proteins $\mathrm{OmpC}$ (during high osmolarity) and $\mathrm{OmpF}$ (during low osmolarity) is crucial in a changing environment ${ }^{84}$.

As well as general survival of the cell in harsh conditions, pathogenic bacteria also

use these signal systems to express virulence factors, some of which are shown ${ }^{89}$ in table 1.1. 


\begin{tabular}{lll}
\hline TCS & Species & Response \\
\hline PilS/PilR & P. aeroginosa & Pilus production \\
FleS/FleR & P. aeroginosa & Adhesion \\
KinA/KinB/SpoF & B. subtilis & Sporulation \\
PhoP/PhoQ & S. typhimurum & Pathogenesis \\
PhoP/PhoR & E.coli & Phosphate sensing \\
\hline
\end{tabular}

Table 1.1: Virulence and survival associated TCS in prokaryotes. (as reviewed by Fontan $2004^{89}$ )

Another concern is the ability of certain TCS to induce drug resistance in a pathogen. Polymixin resistance in Salmonella typhimurium for example is aided by its PhoP/PhoQ TCS, which senses membrane disturbances and activates lipid A modifications to evade to the effects of the drug on the cell ${ }^{95}$. Another example is $E$. coli's ability to alter gene expression under the control of the OmpF/ OmpC system which mediates entry of tetracycline to the cell ${ }^{95}$. Vancomycin resistance is seen in Enterococcus faecalis and Staphylococcus aureus as a result of the VanS/ VanR and VncS/ VncR systems respectively ${ }^{95,96}$. Vancomycin binds to precursors in peptidoglycan synthesis preventing cell wall assembly ${ }^{96}$. The VanS/ VanR system is able to modify peptidoglycans giving them a lower affinity for the drug ${ }^{97}$.

The fact that various TCS have been deemed essential to the organism is another reason they would make potential drug targets; to date Cck/ CtrA of Caulobacter crescentus, MtrB/ MtrA of Mycobacterium tuberculosis, HP166/ HP165 of Helicobacter pylori and YycG/YycF of Bacillus subtilis and Streptococcus pneumonia are considered to be essential ${ }^{98}$. 


\subsection{Two Component Systems in mycobacteria}

The M. tuberculosis genome encodes 11 TCS and seven orphan HKs and RRs ${ }^{86}$. All of these belong to either the OmpR or NarL family of TCS defined by the residues surrounding their $\mathrm{H}$ box. $M$. bovis shares all of these TCS while the M. smegmatis genome contains five out of the seven orphan $\mathrm{HK}$ and RR and nine out of the eleven complete TCS ${ }^{86}$. Table 1.2 names all of these systems and their M. smegmatis homologues with any known conditions regarding their expression, based on articles by Tyagi, Fontan and Haydel ${ }^{113,116,125}$. These TCS are found in a majority of mycobacterial species, with the exception of $M$. leprae which has only 4 out of 12 ; SenX3/ RegX3, PrrB/ PrrA, MprA/ MprB and MtrB/ MtrA.

Some of the TCS of M. tuberculosis have been well studied and their activating factor or factors proposed. Others have been shown to be upregulated during intramacrophage infection, indicating an important function during infection and latency, but the specific condition they are responsive to remains elusive. The systems used in this study were MtrB/ MtrA, DevS/ DevR and SenX3/ RegX3 which will be described.. 


\begin{tabular}{|c|c|c|c|c|}
\hline Name (HK/ RR) & Gene & $\begin{array}{l}\text { M.Smegmatis } \\
\text { homologue }\end{array}$ & Similarity & $\begin{array}{l}\text { Proposed } \\
\text { function }\end{array}$ \\
\hline \multirow[t]{2}{*}{ SenX3/ RegX3 } & Rv0490/ & MSMEG_0936 & 0.695 & Phosphate \\
\hline & Rv0491 & MSMEG_0937 & 0.933 & starvation \\
\hline \multirow[t]{2}{*}{ KdpE/ KdpD } & Rv1028/ & MSMEG_5395 & 0.773 & Nutrient \\
\hline & Rv1027 & MSMEG_5396 & 0.858 & starvation \\
\hline \multirow{2}{*}{ MprB/ MprA } & Rv0982/ & MSMEG_5487 & 0.763 & \\
\hline & Rv0981 & MSMEG_5488 & 0.9 & \\
\hline \multirow[t]{2}{*}{ MtrB/ MtrA } & Rv3245/ & MSMEG_1875 & 0.828 & Iron \\
\hline & Rv3246 & MSMEG_1874 & 0.969 & starvation \\
\hline \multirow[t]{2}{*}{ PrrB/ PrrA } & Rv0902/ & MSMEG_5663 & 0.813 & \\
\hline & Rv0903 & MSMEG_5662 & 0.932 & \\
\hline \multirow[t]{2}{*}{ TrcS/ TcrR } & Rv1033/ & MSMEG_2916 & 0.715 & \\
\hline & Rv1032 & MSMEG_2915 & 0.507 & \\
\hline \multirow[t]{2}{*}{ DevS/ DevR* } & Rv3133/ & MSMEG_5244 & 0.852 & Hypoxia, \\
\hline & Rv3132 & MSMEG_5241 & 0.664 & nitric oxide \\
\hline \multirow[t]{2}{*}{ PhoP/ PhoR } & Rv0758/ & MSMEG_5870 & 0.798 & \\
\hline & Rv0757 & MSMEG_5872 & 0.927 & \\
\hline \multirow[t]{2}{*}{ TcrY/ TcrX } & Rv3765/ & MSMEG_4989 & 0.589 & \\
\hline & Rv3764 & MSMEG_4990 & 0.859 & \\
\hline \multirow{3}{*}{$\begin{array}{l}\text { NarL/ NarS } \\
\text { (NarL family) } \\
\text { TcrA/-/- }\end{array}$} & Rv0844/ & MSMEG_0105 & 0.781 & \\
\hline & Rv0845 & - & & \\
\hline & Rv0600-Rv0602 & - & & \\
\hline \multirow{2}{*}{$\begin{array}{l}\text { Orphan RR, } \\
\text { NasT } \\
\text { Orphan RR }\end{array}$} & Rv1626 & MSMEG_3246 & 0.872 & \\
\hline & Rv0260c & MSMEG_0432 & 0.747 & \\
\hline Orphan RR & Rv0818 & MSMEG_5784 & 0.747 & \\
\hline Orphan RR & Rv3143 & MSMEG_2064 & 0.736 & \\
\hline Orphan RR & Rv2884 & - & & \\
\hline Orphan HK & Rv3220 & MSMEG_1918 & 0.757 & \\
\hline Orphan HK & Rv2027c & - & & \\
\hline
\end{tabular}

Table 1.2: HKs and RR of M. tuberculosis. (All HKs belong to the OmpR family except * which is a NarL type HK)

\subsection{MtrB/ MtrA}

The first TCS to be discovered in M. tuberculosis was the MtrB/MtrA system ${ }^{99}$. Curic et al used a xylE promoter fusion reporter system to show that the mtrA gene expression varied depending on the media used to culture $M$. smegmatis and $M$. bovis ${ }^{99}$. Another group used a Green Fluorescent Protein (GFP) promoter fusion to 
show this gene was expressed during macrophage infection with M. bovis BCG ${ }^{127}$ which was later complemented by work from Via and co-workers ${ }^{100}$. One interesting quality of this TCS is the fact that it has not been knocked out, indicating that it may be essential to $M$. tuberculosis ${ }^{101}$. This has however made it more difficult to investigate its function during infection. In 2006 a group over expressed the MtrA RR showing that overproduction of this protein prevented multiplication during macrophage infection, but not in broth ${ }^{102}$. They concluded that the ratio of phosphorylated to unphosphorylated MtrA was vital for intra macrophage replication, since simultaneously upregulating its $\mathrm{HK}$ MtrB had no effect ${ }^{102}$. They supported this theory by showing that MtrA binds to the promoter of a replication gene $d n a A^{102}$. They also suggested that it may be involved in the AFB's ability to block phagolysome fusion. Friedland et al solved the crystal structure of MtrA and demonstrated that it was similar to the PrrA RR and may have multiple conformations ${ }^{103}$.

Studies of this RR in other species may also give clues as to its function in $M$. tuberculosis. A study of $M$. leprae cell wall associated proteins unearthed an $m$ trA homologue in this species, highlighting its importance for intracellular growth ${ }^{104}$. In M. avium the mtrB locus has been knocked out using transposon mutagenesis, resulting in a strain with attenuated virulence which cannot make the red white switch on Congo red agar ${ }^{105}$. In this species the TCS is required for the control of cell surface proteins and regulates permeability and composition of cell wall components ${ }^{105}$. In a more distantly related species Cornebacterium glutamicum an MtrA knockout showed an effect on antibiotic susceptibility and osmo-regulation of the cell ${ }^{106}$. In this species the mtrA gene is probably involved in peptidoglycan metabolism and osmotic protection ${ }^{106}$. Interestingly, a similar TCS the AmrA/ AmkA 
system of Amycolatopsis mediterranei has been knocked out showing a $20 \%$ decrease in RIF production by the organism ${ }^{107}$.

MtrB/ MtrA is well conserved in other mycobacterial species including M. smegmatis, which makes this system easy to manipulate for this study. Since MtrB/ MtrA appears to be induced during macrophage entry and has not yet been knocked out, this system shows great potential as a drug target in $M$. tuberculosis.

\subsubsection{SenX3/ RegX3}

The SenX3/ RegX3 TCS has been well characterised in M. tuberculosis ${ }^{135-137}$. It is involved in the phosphate transport system and induces the M.smegmatis phoA gene, whose protein product is an alkaline phosphatase required for transport of inorganic phosphate into the cell ${ }^{108}$. It is proposed that this system controls the regulation of around 50 genes as well as the regX3 gene product ${ }^{109}$. This positive autoregulation of the RR is important in TCS for signal amplification ${ }^{110}$. The phoA gene is present in M. smegmatis and $E$. coli but not $M$. tuberculosis ${ }^{111}$. This gene is involved in inorganic phosphate transport in E. coli, a mechanism which has been well characterised in this species ${ }^{135,139,140 .}$. PhoA is induced by SenX3/ RegX3 in $M$. smegmatis and by PhoP/ PhoR in E.coli in a phosphate dependent manner ${ }^{135,139}$. It functions as a cell surface associated lipoprotein which liberates inorganic phosphate from macromolecules ${ }^{111}$.

The phosphate specific transporter PST and phosphate intake transporter PIT are responsible for maintaining cellular levels of inorganic phosphate ${ }^{111}$. The PST proteins act as a sensor of phosphate concentration, keeping RegX3 in an unphosphorylated state ${ }^{108}$. When phosphate is limited the PST complex causes 
dimerization of the SenX3 HK, which activates the RegX3 RR to induce phoA and other genes required for phosphate production ${ }^{108}$.

To summarise, this TCS is implicated in the phosphate transport system, its expression is increased during early macrophage infection ${ }^{112}$ and its knockout displays attenuation of growth in a mouse and macrophage model. This suggests that this TCS may be an important virulence factor ${ }^{109}$. Specific sequence differences between virulent and avirulent strains of the M. tuberculosis complex species have been shown in the sequences of this TCS, indicating that virulence phenotypes may be affected by SenX3/ RegX3 ${ }^{113}$.

\subsubsection{DevS/ DevR}

The DevS/ DevR system is responsible for inducing the dormancy response of $M$. tuberculosis during hypoxia and nitric oxide stress ${ }^{71,72,142-144}$ making it a valuable target in preventing latency ${ }^{114}$. Unlike most other TCS of M. tuberculosis, the DevS/ DevR system is part of the NarS/ NarL family ${ }^{89}$. The DevS HK contains two GAF domains in its sensing core named GAF $A$ and GAF $B^{115}$. It has been proposed that GAF A binds to heme which changes from an oxygenated to a deoxygenated state during oxygen starvation, this results in DevR activation and transcription of a number of dormancy related genes ${ }^{115}$. This assumption was based on the observation that cyanide, which binds with heme, hinders the dormancy response in M. tuberculosis usually induced by nitric oxide stress and oxygen starvation. The importance of this TCS during hypoxic stress has been illustrated by its knockout mutant, which cannot survive oxygen starvation and is more sensitive to heat shock 62. As previously mentioned, the granumloma formed in the human lung during $M$. 
tuberculosis infection leaves the AFB in a hypoxic environment ${ }^{116}$. It is proposed that this oxygen based sensor could be a switch from aerobic to anaerobic metabolism.

A mouse model has shown knockouts of this system to be hyper virulent ${ }^{112}$ while rabbit and guinea pig models show it is required for persistence ${ }^{117}$. Converse et al ${ }^{117}$ showed marked differences in results depending on the model host used.

Considering the different pathology shown in different host organisms, it is possible the DevS/ DevR system is required to shut down active metabolism and therefore the knockout appears hyper virulent in mice because this host does not naturally show latency.

During the M. tuberculosis dormancy period a number of genes required for active metabolism are repressed, while stress response genes, including $h s p X$, are induced $47,144,148$. The $h s p X$ gene product is an alpha crystallin antigen which may play a role in stabilizing proteins during persistence ${ }^{63}$. As well as $h s p X$, DevS/ DevR induces 3 universal stress proteins (UspL,M and N) and a nitroreductase (Rv3131) ${ }^{118}$.

The signal transduction mechanisms of $M$. tuberculosis undoubtedly have an overall effect on its virulence and pathogenesis, in particular the switch from active to latent infection. It is in this light that these systems are viewed as excellent potential drug targets in mycobacteria and other pathogenic prokaryotes. Consequently, a number of groups have attempted to develop inhibitors to these signalling systems. 


\subsubsection{Two Component Systems as Drug Targets}

The search for new anti-TB drugs is a particularly important component of the STOP TB strategy, given the rising threat of MDR and XDR-TB. Using growth inhibition assays to discover anti-tubercular agents from a vast array of compounds by measuring the effect on the whole cell, may lead to the discovery of effective agents against the pathogen. On the other hand, a more targeted approach to specific components of the cells dormancy program is more likely to yield an agent with a detrimental effect on $M$. tuberculosis during a latent TB infection.

Since the discovery of TCS and their inferences to virulence and persistence, they have been labelled as critical targets in pathogenic bacteria ${ }^{98}$. Generally speaking signal transduction mechanisms allow bacteria to persist and cause disease in a human host; TCSs in particular are of great interest for four main reasons ${ }^{98 .}$

- Their ubiquity among prokaryote organisms and selective presence in higher eukaryotes, indicates a TCS inhibitor would be less likely to exert a negative effect on a human host

- $\quad$ Principle active sites in TCS display a high level of homology, so a TCSi could potentially act on a number of TCS across a broad spectrum of bacteria

- $\quad$ At least four TCS are essential to their host organisms

- $\quad$ TCS are often used by prokaryotes to regulate the expression of virulence factors

More specifically, in terms of $M$. tuberculosis, current drug targets act on processes such as cell wall and protein assembly, which are only effective in the case of an active TB infection. In targeting the mechanisms $M$. tuberculosis employs to sustain 
an ongoing latent infection, problems encountered with many of the current anti-TB agents such as toxicity, lengthy treatment duration and the emergence of resistant strains could be prevented ${ }^{114}$. 


\subsection{Inhibitors of TCS}

There are multiple stages of the TCS phosphorelay that could be targeted to inhibit this type of signal transduction ${ }^{119}$. Figure 1.3 shows some of the reactions of the phosphorelay which could potentially be inhibited as well as examples of known TCS inhibitors and their proposed targets. In theory, targeting a conserved mechanism such as the phospho transfer between the histidine and aspartate would give rise to a broad spectrum inhibitor. On the other hand, targeting a specific ligand binding mechanism of the HK, or DNA binding of the RR, would render TCSi that is fairly specific to that TCS.

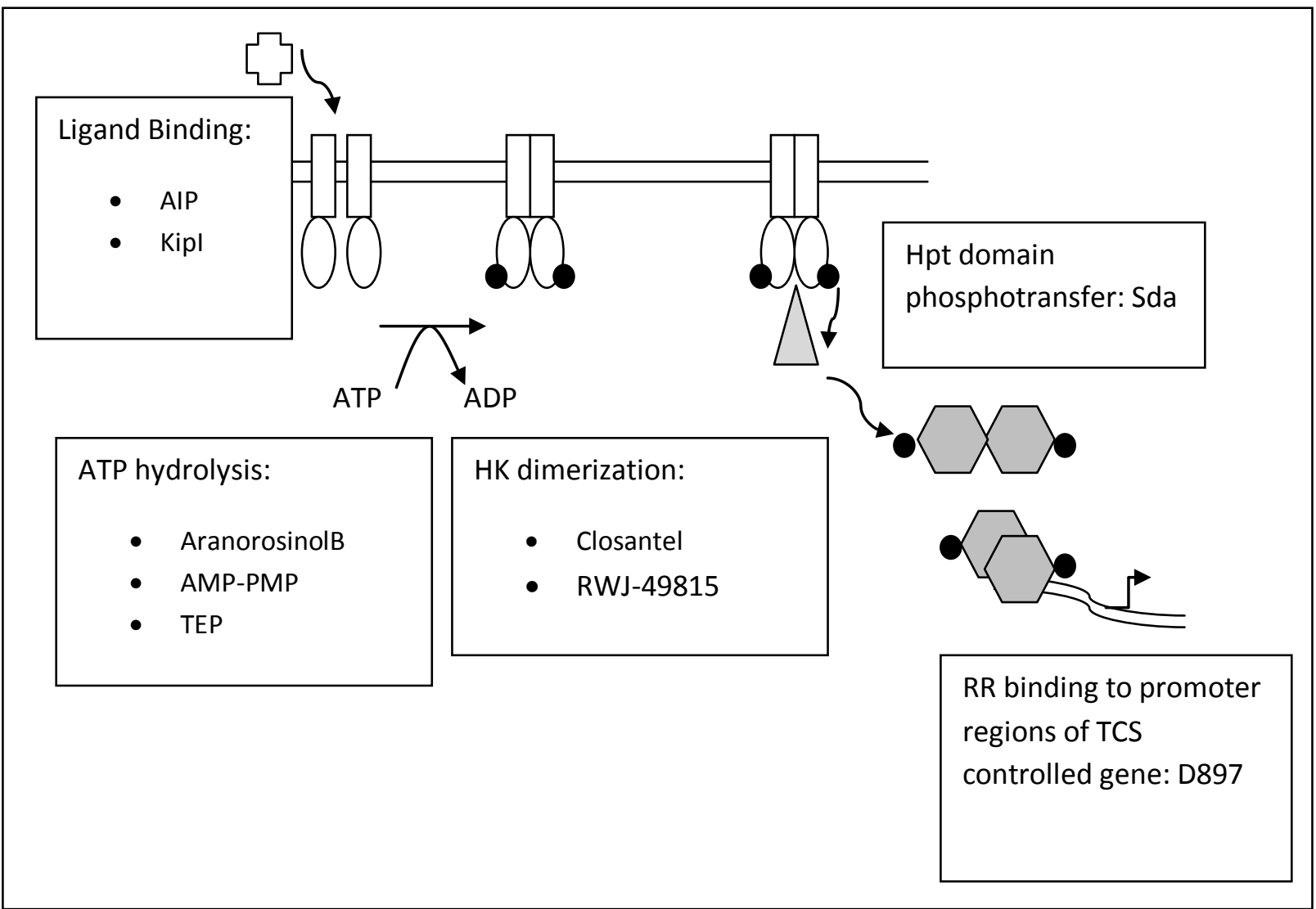

Figure 1.3: TCS Inhibitors. A summary of known TCSi and their proposed mechanisms of action. Agents have been found which inhibit a number of the steps involved in TCS signal transduction including ligand binding to HK, ATP hydrolysis, HK dimerization and RR phosphotransfer and promoter binding. 
To date there have been a number of compounds introduced to the field which act on specific components of the signal transduction machinery, but none that do so in a way that affects signalling independently of other prokaryote and eukaryote cellular processes $^{98}$.

\subsubsection{Inhibition of the Histidine Kinase}

Compounds which affect ligand binding to HK sensor domains have been developed for specific targets ${ }^{98}$. These are usually derivatives of naturally occurring ligands which are modified at specific residues in order to compete with naturally occurring ligands of the TCS.

Streptomyces produce autoinducing peptide (AIP) to induce virulence factor Agr required for quorum sensing ${ }^{119}$. When the population reaches a threshold density, secretion of AIP binds the HK of the ArgC/ ArgA TCS effectively inducing virulence genes and repressing surface proteins ${ }^{119}$. Replacing the sulphur of the thiolactone ring structure of AIP with $\mathrm{O}$ or $\mathrm{N}$ provides a competitive inhibitor of their quorum sensing mechanism ${ }^{119}$.

Kipl is a protein inhibitor of $B$. subtilis sporulation TCS KinA/SpoOA ${ }^{120}$. This protein inhibits the autophosphorylation of KinA, but not the phospho transfer step. Another gene in its operon, kipA, counters its activity ${ }^{120}$.

A number of chemicals have been found to inhibit the ATP to ADP hydrolysis shown in figure 1.4. These can act in either a competitive or non competitive fashion. AMPPNP (adenosine monophosphate p-Nitrophenol), TEP (triethyl phosphate) and aranorosinol B for instance competitively inhibit this hydrolysis reaction while cis 
unsaturated fatty acids such as oleic and palmitoleic act in a non competitive fashion ${ }^{98}$. While they effectively inhibit this signal transduction process, they would clearly affect a number of other processes in both the host and pathogen.

Most TCS inhibitors to date interfere with the dimerization domain of a HK, exposing hydrophobic amino acids resulting in an aggregation of $\mathrm{HKs}$ at the cell membrane 121. Closantel and RWJ-49815 act in this way, denaturing the dimer of KinA by interfering with its 4 helix bundle between the sensor and effecter domains ${ }^{122}$. Without suitable dimerization, signal transduction cannot be relayed to the RR. These compounds have the same effect in a sensor kinase in E. coli reflecting a broad spectrum of activity ${ }^{96}$. Unfortunately, many of these HK dimerization inhibitors also have an effect on membrane intergrity in both bacterial and mammalian cells and thus have not been developed as antibiotics.

The Sda protein inhibits the sporulation system KinA/KinB through binding of the histidine phosphotranfer domain (HPt) of KinB ${ }^{121}$. This compound binds with the alpha helices, not the conserved $\mathrm{H}$ box or catalytic domain, and acts as a molecular barricade, hindering communication between $\operatorname{KinB}$ and $\operatorname{KinA}^{121}$.

\subsubsection{Inhibition of Response Regulator}

A less common mechanism of action is the inhibition of the RR activity. A modified protein can alter a HK/ RR interaction via competition for their binding site. Modifications to the DrrA peptide of the HpkA/DrrA system in Thermotoga maritima caused competitive binding for HpkA resulting in a loss of dimer formation ${ }^{123}$. A compound named D897 is able to inhibit DNA binding of RR to the promoter regions

of induced genes by preventing the dimerization of RR required for DNA binding ${ }^{98}$. 
Although natural and synthetic compounds have been shown to inhibit stages of this process, research in the field has yet to provide a compound which affects these processes exclusively. A major shortcoming of many TCS inhibitors described is an adverse effect on membrane integrity of both bacterial and human cell membranes 98. A study of 24 TCS inhibitors showed that $23 / 24$ had adverse effects on membrane integrity. This suggests that their activity may be the result of another mechanism altogether, such as membrane interference or macromolecule synthesis ${ }^{124}$. Unfortunately the only compound free of this defect had a relatively high minimum inhibitory concentration (MIC) ${ }^{124}$. Other TCS inhibitors exhibit severe toxic side effects, closantel for example adversely affects energy metabolism in the mitochondria $^{125}$.

That being said, the organism Staphylococcus epidermis causes major problems with its ability to form biofilms on medical instruments ${ }^{126}$. It has been suggested that an inhibitor of the YycG HK of this organism, required for biofilm formation, could easily be used as a sterilization agent on medical equipment if it were cost effective 126. Another suggestion is the use of non toxic HK inhibitors in combination with other drugs to improve a therapeutic outcome. Isothiazolone and imidazolin salts were the first TCS inhibitors to be discovered ${ }^{127}$. These aromatic structures showed activity against the AlgR2/ AlgR1 system, an important virulence factor of $P$. aeroginosa which causes major problems in cystic fibrosis patients ${ }^{127}$. These could be used to improve the therapeutic outcome of patients with cystic fibrosis ${ }^{98}$ even though they do no inhibit the organism outright. Despite work by several groups to discover and develop TCS inhibitors, none been made commercially available as antibiotics or bacteriostatic agents in a medical context. The aim of this study was to develop a way in which one can screen for novel TCSi, which inhibit mycobacterial signal 
transduction exclusively of other cellular processes, by incorporating a whole cell based approach as opposed to inhibiting individual proteins.

\subsubsection{Screening for TCS inhibitors}

High throughput screening (HTS) methods allow researchers to test thousands of compounds at a time for activity against TCS. There are two approaches here, either random screening of a large number of compounds from a library ${ }^{119}$ or rational design using virtual screening methods based on the crystal structure of a target protein ${ }^{126}$. The latter method allows one to screen a much larger database of compounds, however all hits must be validated by another method to show that the compound specifically inhibits the putative target in vitro.

A direct approach involves the use of purified, truncated or altered HK to screen for specific inhibitors. Inhibition can be demonstrated using radio labelled phosphate as a donor in the ATP dependent autophosphorylation of purified HKs and RR ${ }^{98,128 .}$ This sort of assay has been established by Saini et al with M. tuberculosis DevS /DevR system proteins ${ }^{128}$. The Taz-1 assay was used to find inhibitors of the EnvZ/ OmpR system ${ }^{129}$. In this screen the HK receptor signal is replaced by an aspartate chemoreceptor which stimulates autophosphorylation of the HK in the presence of aspartate. A lacZ promoter fusion of the ompC gene was also used to determine OmpR binding to this DNA region ${ }^{129,130}$. The homodimerization assay exploits the capacity of certain truncated HKs to dimerise in vitro ${ }^{98}$. Using a plasmid with GFP under the control of the E. coli iclR promoter, truncated Yyc proteins expressed with the IcIR repressor can only induce GFP expression when present as a dimer ${ }^{98}$. 
These sophisticated screening methods are limited in that they deal with altered forms of proteins which do not naturally occur in the cell. Using the native forms of these proteins in a whole cell based assay may deliver TCS inhibitors which represent a higher level of efficiency in a whole cell model.

Whole cell based methods have a number of advantages over isolated protein methods . For one thing, TCS inhibitors found using protein based methods may be potent inhibitors of signal transduction, but their ability to permeate the cell membrane is not taken into account. What is more, other metabolic activities of the cell are accounted for, such as proteins and enzymes which could decrease the activity of these compounds. As previously mentioned, all of the current known TCSi have failed in that they cause adverse affects on bacterial and mammalian cell walls. Many bacterial species also have efflux pumps which could potentially remove these inhibitors from the cell before it reaches its target.

Another consideration is the specificity of the target. Using isolated protein methods confines the researcher to finding inhibitors of a specific molecule or reaction in the chain of events involved in signal transduction. Using a whole cell based method, which measures the end result of the signalling mechanism, i.e. the up-regulation of a particular gene under the control of that system, increases the likelihood of an inhibitor of any part of this phosphorelay being picked up in the screen.

In 1993 Roychoudhury et al discovered the first TCSi by screening around 25,000 synthetic compounds using a whole cell $x y / E$ reporter system assay ${ }^{127}$. Inhibition of the RR binding activity to the algD promoter was demonstrated in 15 compounds using this colourmetric assay ${ }^{127}$. Differential growth assays are another method that 
can be applied to whole cell screening. Temperature sensitive mutants of the YycG HK showed hypersensitivity to 11 compounds in a screen of 4000 acetone extracts 131. These hits were further characterised, leading to the detection of aranorosinol B's ability to inhibit the autophosphorylation of YycG in S. aureus and B. subtilis ${ }^{131}$.

Measuring expression of a TCS target gene is a valuable method because it accounts for everything that is going on in the cell at once. These methods show that a compound is capable of reaching its target and that gene expression is being inhibited in spite of whatever else is going on in the cell. However, it is an indirect method of detecting TCS inhibition. Usually validation either at a protein level or by measuring the expression of an unrelated gene is required in order to show that a compound does not simply have a global effect on gene expression. It is also important to consider any implications that reporter systems may have on both the cell and compounds in the screen and vice versa.

In this study a GFP reporter was used to measure the expression of promoters under the control of mycobacterial TCS to evaluate conditions which induce these systems and screen for TCS inhibitors using available drug libraries.

\subsubsection{Methods used in this Study}

The aim of this study was to apply a promoter reporter method to demonstrate appropriate conditions under which selected TCS genes are induced, so they can be used in a screen for two component system inhibitors. GFP, isolated from Aequorea victoria, has been used to monitor gene expression since $1994^{132,133}$. It is an ideal reporter as it does not require substrate activation ${ }^{133}$ and numerous enhanced variants of the original wildtype GFP exist for specific purposes ${ }^{134-136}$. GFP mut2 will 
be used to scrutinise the expression of TCS-regulated genes, and examine changes that occur to these expression profiles as a result of exposure to compound libraries. The constitutively expressed $h s p 60$ promoter will be included to verify that a shift in GFP fluorescence is the result of some mechanism other than a reduction in global gene expression on the cell. The model organism M. smegmatis will be used so that these assays can be carried out to test as many compounds as possible in a high throughput manner as it grows more rapidly and can be safely manipulated in a PC2 laboratory. The resultant experimental procedure could lead to further studies of the more pathologically relevant $M$. bovis BCG once the assay is optimised for $M$. smegmatis. 


\subsection{Aims}

The overall objective of this research was to design a screen which identifies inhibitors of mycobacterial TCS related to dormancy and intra macrophage survival. This required four main objectives to be carried out:

1. Create a list of suitable M. tuberculosis and M. smegmatis TCS dependent genes and identify their promoter sequences.

2. Clone these promoter sequences and an optimised GFP reporter gene into a plasmid which can be electroporated into M. smegmatis.

3. Demonstrate conditions under which the greatest expression of these promoters is found.

4. Develop a bioassay to screen compounds for mycobacterial TCS inhibitors based on the expression of these TCS regulated genes. 


\section{CHAPTER TWO:}

\section{Materials and Methods}




\subsection{Strains and culture conditions}

The plasmid construction was carried out in E. coli DH5a grown in Luria Broth (LB) or on Luria agar (LA) from Sigma Aldrich, with either kan ${ }_{50}$ (kanamycin $50 \mu \mathrm{g} / \mathrm{ml}$ ) or amp $_{200}$ (ampicillin $200 \mu \mathrm{g} / \mathrm{ml}$ ). M. smegmatis $\mathrm{MC}^{2} 155$ was growth on LA or in LB supplemented with $0.1 \%$ Tween 80 and $1 \%$ D- arabinose or Hartmans deBonts (HdeB) minimal media as described ${ }^{53}$ using kan ${ }_{50}$ for plasmid selection. Further modification to culture media for stress condition assays shall be discussed in section 2.6.

\subsection{Plasmids and Primers}

This work involved the use of a number of published and unpublished plasmids from various sources as well as the creation of a new pSHigh construct. The plasmids are briefly described and their sources displayed in table 2.1. Primers used to clone and sequence elements of the new reporter construct are displayed in table 2.2.

\begin{tabular}{|c|c|c|}
\hline Plasmids & Description & Source \\
\hline pcr 2.1 & TA cloning vector, $\mathrm{Amp}^{r}, \mathrm{Kan}^{\mathrm{r}}$ & Invitrogen \\
\hline pLUG & TA cloning vector, $\mathrm{Amp}^{r}$ & Bioline \\
\hline pTKmx & pAL500, pUCori, Kan', xylE & 137 \\
\hline pHS201 & $\mathrm{P}_{\text {hsp60, }}$, GFPmut2, oriM high, pUCori, Kan ${ }^{r}$ & 138 \\
\hline pOT11 & $P_{\text {tet, }}$ GFPmut2, pMMB207 backbone & 139 \\
\hline pOT71 & $P_{\text {hsp60 GFPmut2, pAL5000, pUCori, Kan }}{ }^{r}$ & O'Toole (unpublished) \\
\hline pOT72 & $\mathrm{P}_{\text {hsp60 }} \mathrm{HSP60}$, GFPmut2, pAL5000, pUCori Kan ${ }^{r}$ & O'Toole (unpublished) \\
\hline pOT62 & $P_{\text {hsp60, }}$ GFP RBS, GFPmut2, pAL5000, pUCori, Kan ${ }^{r}$ & O'Toole (unpublished) \\
\hline pSHigh & GFP RBS, GFPmut2, oriM high, pUCori, Kan' & This study \\
\hline pHigh100 & GFPmut2, oriM high, pUCori, Kan ${ }^{r}$ & 138 \\
\hline
\end{tabular}

Table 2.1: Plasmids used in this study. 


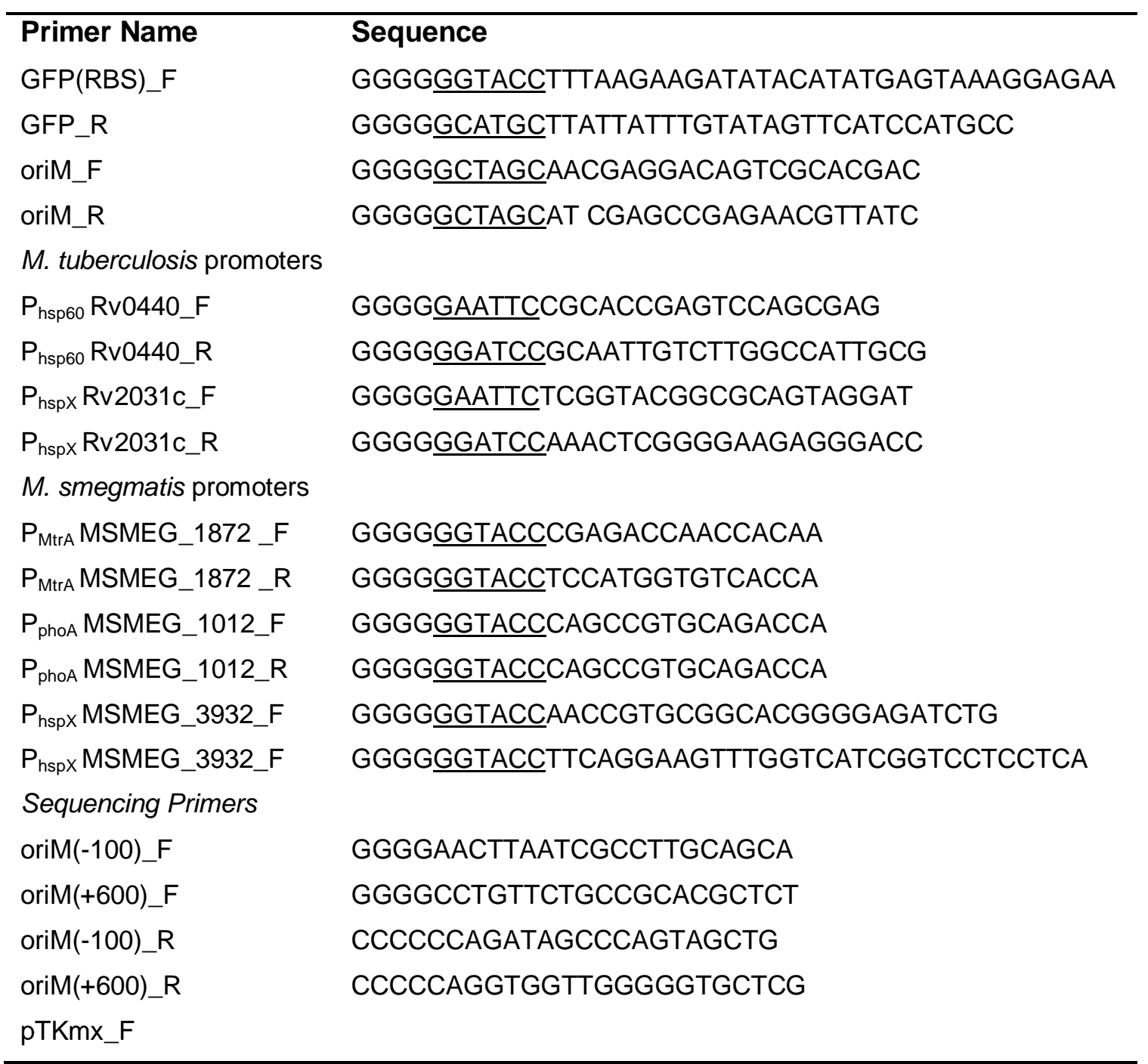

Table 2.2: Primers used in this study. Restriction sites of primers are underlined

\subsection{Identification of TCS dependent genes in M. tuberculosis and}

\section{M. smegmatis}

A list of candidate M. tuberculosis and M. smegmatis TCS controlled genes was established after a literature search based on the following criteria: 
1. The candidate must contain a non-coding region upstream of the gene or have its promoter region identified, preferably with a gel mobility shift assay consistent with the ability to bind directly with a RR.

2. Factors or conditions inducing expression of the gene should be postulated or proven.

3. A high fold induction of gene expression upon stimulation, or a significant decrease in expression of that gene displayed in the TCS mutant.

Sequences of candidate genes and their organisation within an operon were obtained from www.genome.jp. A promoter region of approximately 600 base pairs was selected directly upstream of each candidate gene for primer design.

\subsection{Plasmid Manipulation}

Cloning procedures were carried out with T4 ligase from Bioline and restriction enzymes and their respective buffer solutions from New England Biolabs (NEB). Polymerase chain reactions (PCR) were carried out with Taq polymerases from either Bioline or Invitrogen limited.

\subsubsection{Amplification of Promoters from Genomic DNA}

Genomic DNA (gDNA) from heat treated $M$. tuberculosis H37Rv was provided by AgResearch (Upper Hutt). M. smegmatis gDNA was prepared by standard genomic

preparation as described by Sambrook ${ }^{140}$. Primers were designed to amplify around 600 base pairs of a promoter region upstream of each gene of interest, including at least the start codon of each gene. Primers contained EcoRI and BamHI restriction sites for cloning into low copy number POT constructs, or Kpnl for cloning into 
pSHigh constructs. In addition, the highly conserved promoter of the hsp60 gene (Rv0440) was cloned as a constitutively expressed positive control. Promoters were amplified from gDNA using the high fidelity polymerase Platinum Taq (Invitrogen Ltd.) according to manufacturer's instructions with an Eppendorf thermocycler. Appendix 1 displays a summary of all PCR conditions used in this study. PCR products were visualised on a $1 \%$ agarose gel to check they were the correct size, before the PCR products were isolated using a clean and concentrate kit from Zymo Ltd.

\subsubsection{Ligation of promoters into Cloning Vectors and heat shock Transformation into E.coli}

Purified PCR products were ligated into either pCR2.1 of pLUG TA cloning vectors using T4 ligase by incubating promoter, vector, ligase enzyme and $1 \mathrm{x}$ ligase buffer for at least 4 hours at $16{ }^{\circ} \mathrm{C}$. A summary of ligation reactions in this study is shown in appendix 2.

Ligation reactions were then transformed into calcium competent $E$. coli $\mathrm{DH} 5 a$ by heat shock transformation. For notes on preparation of calcium competent DH5a see appendix 4 . The optimal ratio of cells to ligation product is $10: 1$ so for a $10 \mu \mathrm{L}$ ligation reaction, aliquots of $100 \mu \mathrm{L}$ of $\mathrm{DH} 5 \alpha$ cells were used. Cells were first thawed on ice for 5 minutes before adding the ligation product. Following a 20 minute incubation period on ice, cells were heat shocked at $42{ }^{\circ} \mathrm{C}$ in a heat block for 2 minutes and then returned to ice for 5 minutes. The cells were then recovered at $37^{\circ} \mathrm{C}$ without agitation, for at least 45 minutes in $600 \mu \mathrm{L}$ of LB, without antibiotics, to allow expression of the antibiotic cassettes contained on the plasmid. Following recovery, 
cells were centrifuged for 30 seconds at $12000 \mathrm{rpm}$ and the supernatant removed to leave $100 \mu \mathrm{L}$ of cells which were re-suspending by gentle pipetting.

For blue white colony selection, cells were spread on Luria agar with Isopropyl-Betad-Thiogalactopyranoside (IPTG) at $100 \mu \mathrm{g} / \mathrm{ml}$, X-galactosidase (Xgal) at $40 \mu \mathrm{g} / \mathrm{ml}$ and ampicillin at $200 \mu \mathrm{g} / \mathrm{ml}$ and incubated at $37^{\circ} \mathrm{C}$ overnight. At least 5 white colonies for each ligation reaction were selected and inoculated in $3 \mathrm{~mL}$ overnight cultures of LB with ampicillin $(200 \mu \mathrm{g} / \mathrm{ml}) .600 \mu \mathrm{L}$ from each culture was reserved for a $-80{ }^{\circ} \mathrm{C}$ freezer stock, made with an equal volume of $80 \%$ glycerol to give a final concentration of $40 \%$ gylcerol.

\subsubsection{Plasmid Isolation and Digestion}

The remainder of the liquid culture was used for plasmid preparation. In this study plasmid DNA was isolated using either alkaline lysis with phenol chloroform extraction as described ${ }^{140}$ or using a Zymo plasmid preparation kit according to manufacturer's instructions. The pellet free Zymo kit method was preferred when plasmids were to be sequenced or electroporated, since this method gives a cleaner plasmid product. The alkaline lysis method was used when a concentrated DNA preparation was required, for example before removal of a fragment by restriction digest.

The presence of the inserted promoter fragment was verified with a small amount of the plasmid preparation by a restriction enzyme digest with EcoRI. A summary of restriction enzyme digestions can be found in appendix 3 . EcoRI sites surrounding the cloning region of the TA vector result in a drop-out of the fragment after digestion giving two distinct bands on a $1 \%$ agarose gel. Plasmid preparations which displayed this fragment drop out were sequenced by the Alan Wilson Centre (AWC) in 
Palmerston North using primers M13 forward and reverse, which are present on the TA cloning vector surrounding the insertion site.

\subsubsection{Sequence Alignment}

Chromatogram data were analysed for fidelity using Vector NTI software (Invitrogen). The sequence displayed between the M13 forward and reverse primers was then aligned with the published sequence of the candidate promoters using AlignX software (Invitrogen). It was important that these sequences were accurate since the exact location of response regulator binding site on the promoter was not always known. In some cases this process was repeated until the sequence of each promoter was identical to the published genome. Clones with correct promoter sequences were identified and used to construct GFP reporter plasmids.

\subsubsection{Low Copy Number Reporter Constructs}

TCS regulated promoters were cloned into low copy number mycobacterial plasmid pOT71 with EcoRI and BamHI restriction enzymes sites. The reporter plasmids were developed based on pTKmx plasmids from Kenney et $a^{137}$. These contain a kanamycin resistance cassette, mycobacterial origin of replication pAL5000, E. coli origin of replication pUCori (also known as oriE) and gfpmut2 cloned from the pKEN plasmid created by Cormack et al ${ }^{136}$ with a multiple cloning site in front of the GFP. Plasmid DNA of the pOT71 plasmid was digested with EcoRI and BamHI with $1 \mathrm{x}$ BamHI buffer overnight at $37^{\circ} \mathrm{C}$. Around $3 \mu \mathrm{L}$ of this reaction was visualised on $1 \%$ agarose gel to ensure a single band was obtained. Restriction enzymes were removed by column purification using a Zymo clean and concentrator kit. 
Sequenced promoters were concurrently digested out of their cloning vectors using the same restriction enzymes. Restriction enzyme reactions were run through a $1 \%$ agarose gel to separate plasmid and promoter fragments. These were then excised and the DNA purified using a Zymo gel extraction kit according to the manufacturer's instructions. To ensure DNA was not lost in this process, the DNA was visualised by running a small amount of the sample on a $1 \%$ agarose gel.

Promoters were then inserted into pOT71 by incubation with T4 ligase and T4 ligase buffer at $16^{\circ} \mathrm{C}$ for at least 6 hours. A small amount of $K p n l$, which cuts between the two cloning sites $(E c o R I$ and $B a m H I)$, was added to the reaction to prevent unwanted uncut pOT72 from remaining in the reaction. The ligation reaction was then transformed into $\mathrm{DH} 5 \alpha$ by heat shock as previously described and plated on LB supplemented with $\mathrm{kan}_{50}$.

The primer pTKmx F1 corresponding to a region on the plasmid about $100 \mathrm{bp}$ upstream of the cloning site, was used in combination with each promoter's reverse primer to screen for clones with the promoter correctly inserted into the pOT71 plasmid. This was carried out by colony PCR screen, according to the manufacturer's guidelines for using Mastermix from Bioline, except that cells from a single colony were used as template DNA, and added using a toothpick or pipette tip. The same tip was then added to a $3 \mathrm{~mL}$ falcon tube containing $\mathrm{LB}$ and kanamycin for an overnight incubation at $37^{\circ} \mathrm{C}$.

The $3 \mathrm{~mL}$ overnight cultures for all positive clones were used to create freezer stocks and plasmid preparations of the POT71 + promoter constructs. These were then 
sequenced using the pTKmx F primer to ensure promoters were correctly inserted in frame with GFP in the plasmid.

\subsubsection{High Copy Number Reporter Constructs}

While this work with the pOT71 plasmids was being carried out, Bourn et al published a paper on a high copy number plasmid for mycobacteria involving the use of a modified pAL5000 origin of replication which will henceforth be known as oriM high ${ }^{138}$. They showed a seven fold increase in plasmid copy number compared with the original pAL5000 origin of replication. A modification of 3 base pairs, causing an alanine deletion, lead to a significant increase in gfp expression on their pHS201 plasmid ${ }^{138}$. The authors kindly gifted us this plasmid to use in our work. Unfortunately their plasmid contained an EcoRI site in front of the GFP, which was also found in their modified oriM so it was impossible to clone promoters directly into this plasmid. A new plasmid was constructed using their modified high copy number origin of replication named pSHigh.

Initial experiments involving the pOT plasmids indicated that pOT62, containing a ribosomal binding site (RBS) upstream of the gfp gene, displayed a higher fluorescent intensity than the pOT71 plasmids. In order to include this RBS sequence in front of the GFP in the new plasmid pSHigh, a forward primer was designed to include this sequence in front of the GFP start codon. We reasoned that having a terminator upstream of the GFP would also improve the vector by avoiding leakage and unwanted GFP expression. The pTKmx plasmid contains a terminator site upstream of the multiple cloning site, so this plasmid was used to create the new high copy number vector. In summary, three improvements were made to optimise the potential of this plasmid as a reporter of gene expression: 
- High copy number origin of replication (oriM high from pHS201).

- Ribosomal binding site for GFP (added to the primer sequence).

- Terminator site upstream of cloning site (trp from pTkmx).

\section{GFP RBS insertion}

The gfpmut2 gene, as well as its RBS, was cloned from pOT11 with GFP_RBS_F and GFP_R primers by PCR using a high fidelity polymerase, Platinum Taq (Invitrogen). As described for the TCS controlled promoters in section 2.3, this gene was cloned into a TA vector, sequenced and then excised with $\mathrm{Kpnl}$ and Sphl. The pTKmx plasmid was also digested with these enzymes to replace the $x y / E$ component of this plasmid with the gfpmut2 by ligation and heat shock transformation onto LB agar with $\mathrm{kan}_{50}$. Screening colonies of $\mathrm{DH} 5 \alpha$ during this step was facilitated by briefly placing the plate on a UV light box and selecting brighter colonies for the PCR screen, which increased the number of positive clones containing the PTKMx_F and Gfp_R primed amplicon. Correct orientation was verified by sequencing with $p T K m \times F$ primer.

pAL5000/ oriM high switch

The original origin of replication was removed from pTKmx by digestion with Nhel and separated from the rest of the plasmid by gel extraction. The high copy oriM high was amplified from Bourn's pHS201 plasmid using primers oriM_F and oriM_R with a modified PCR protocol for amplification of long fragments (see Appendix 1).

The PCR product was visualised on a $1 \%$ agarose gel and then purified using a clean and concentrator column to remove PCR enzymes before ligation into pTKmx -pAL5000. Ligation products were transformed by heat shock into DH5 $\alpha$ and 
screened using oriM-100_F and oriM-100_R primers, which are located $100 \mathrm{bp}$ upstream and downstream of the insertion site. PCR products were visualised on $1 \%$ agarose and positive clones were sequenced by AWC with the oriM -100 primer set to make the plasmid pTkmx_oriM.

\section{Ligation of Promoters}

Promoters were amplified from gDNA with re designed oligonucleotides containing Kpnl sites on both forward and reverse primers. These were ligated and cloned into either pLUG or pCR2.1 TA cloning vectors, sequenced and cut out using Kpnl.

\section{Ligations with one restriction site}

A number of steps were taken to improve the efficiency of these ligations

- A much higher concentration of promoter DNA was obtained by preparation with alkaline lysis instead of using the pellet free plasmid prep kit.

- During gel extraction as much of the unstained agarose (containing no DNA) was removed before the sample was melted in the ADB buffer provided in the kit, to improve the DNA yield.

- The pSHigh plasmid was incubated with Kpnl for at least 15 hours and then run on $0.8 \%$ agarose to visualise the different band size in cut and uncut DNA and separate the two.

- Kpnl cut pSHigh was extracted from the agarose gel and then incubated with calf intestinal phosphatase (CIP) at $37^{\circ} \mathrm{C}$ for 30 minutes; CIP was removed from the sample by column purification before ligation. 
- A pre-ligation heat step involving incubation of plasmid and insert at $65^{\circ} \mathrm{C}$ for ten minutes to break weak bonds between DNA fragments which may have formed dimers.

- A negative control containing SDW instead of the promoter DNA was included. The number of colonies on the negative control plate, containing cut plasmid and SDW rather than insertion fragment, gives an indication of the efficiency of the ligation.

\subsection{Electroporation of constructs into M. smegmatis}

Reporter constructs were transformed into M. smegmatis $\mathrm{mc}^{2} 155$ by electroporation with a Biorad gene pulser. Plasmid preparations were desalted by column purification and quantified by gel electrophoresis because unclean plasmid preparations result in the electroporator arcing. The method for preparation of electro-competent $M$. smegmatis is summarised in appendix 5. Competent cells were thawed on ice for 5 minutes before $200 \mu \mathrm{L}$ of cold $10 \%$ glycerol was added to each $100 \mu \mathrm{L}$ aliquot. Between 7 and $10 \mu \mathrm{L}$ of DNA was added to the cells and glycerol, this solution was mixed by gentle pipetting and moved to a cold electroporation cuvette. Cells and DNA were then stored on ice for at least 2 hours before electroporation. Cuvettes containing cells, DNA and glycerol were pulsed at the following settings according to Mycobacteria Protocols ${ }^{141}$ :

- Voltage $1250 \mathrm{~V}$

- Capacitance $25 \mu \mathrm{F}$

- Resistance $\quad 1000 \Omega$

- Cuvette $2 \mathrm{~mm}$ 
After electroporation, cells were allowed to recover overnight in $600 \mathrm{~mL}$ LB without antibiotics or shaking in eppendorf tubes and then plated on LB kan ${ }_{50}$ to select for plasmid containing clones. M. smegmatis generally takes around 3 days to appear on plates, at which point freezer stocks and overnight cultures were made.

\subsubsection{Visualisation of cells}

Cells were visualised under oil immersion for fluorescence using a microscope with a FITC (Fluorescein Isothiocyanate) filter. Around $500 \mu \mathrm{L}$ of cells were removed from overnight cultures and washed with equal amounts of $1 \times$ PBS. Cells were then spun down for 30 seconds at $12,000 \mathrm{rpm}$ and $400 \mu \mathrm{L}$ of supernatant removed before resuspension. Around $10 \mu \mathrm{L}$ of this concentrated cell solution was used to make slides without staining. 


\subsection{Stress Condition Assays}

\subsubsection{Oxygen Starvation}

\subsubsection{Oxygen Starvation Assay with low Copy number constructs}

A method for growing $M$. smegmatis in oxygen starved conditions has been previously described ${ }^{62}$.

For oxygen starvation, HdeB minimal media was made from a stock of metal salts, 100x nitrates $(\mathrm{N})$, Glycerol and 100x phosphates. $15 \mathrm{~mL}$ overnight cultures were incubated with agitation of $M$. smegmatis containing three different plasmid constructs:

- pOT71 (negative control plasmid containing no promoter)

- pOT71_Rv0440 (positive control promoter containing constitutively expressed hsp60 promoter)

- pOT71_Rv2031 (oxygen sensitive hspX promoter)

Overnight cultures were diluted to an $\mathrm{OD}_{600}$ of 1.0 in $50 \mathrm{~mL}$ HdeB minimal media with $\mathrm{Kan}_{50}$ and grown in $250 \mathrm{ml}$ flasks with airtight rubber seals ${ }^{53}$. Triplicate cultures were grown at $37{ }^{\circ} \mathrm{C}$ with shaking and $1 \mathrm{~mL}$ samples of the culture were removed periodically with a syringe to avoid oxygen being let into the flasks. These samples were measured for $\mathrm{OD}_{600}$ using a spectrophotometer and fluorescence using a Wallac envision plate reader with excitation and emission set at $488 \mathrm{~nm}$ and $509 \mathrm{~nm}$ respectively. 


\subsubsection{Optimised Oxygen Starvation Assay}

Smaller $15 \mathrm{~mL}$ flasks were used with $13 \mathrm{~mL}$ of cells and media using up more of the total volume. A number of identical flasks were set up so that different cultures could be used to measure OD and $\mathrm{F}$ at different time points, without letting air in. Cells in this assay were grown in rich media instead of HdeB and a final concentration of 1.5 $\mu \mathrm{g} / \mu \mathrm{L}$ of methylene blue was added to a fourth tube.

The loss of blue colour in these flasks over 4 days indicated that the level of oxygen was being depleted. In addition, the newly made high copy number plasmid pSHigh was used.

\subsubsection{High throughput stress conditions}

Overnight cultures of each reporter construct were grown and then diluted to an $\mathrm{OD}_{600}$ of $0.2 .50 \mu \mathrm{L}$ of cells were added to equal volumes of media in a $96 \mathrm{wp}$, giving a final cell turbidity of $O D_{600} 0.1$. The 96 wps were set up with a gradient of each stress condition as summarised in figure 2.1 and table 2.3, each plate containing different reporter construct. 


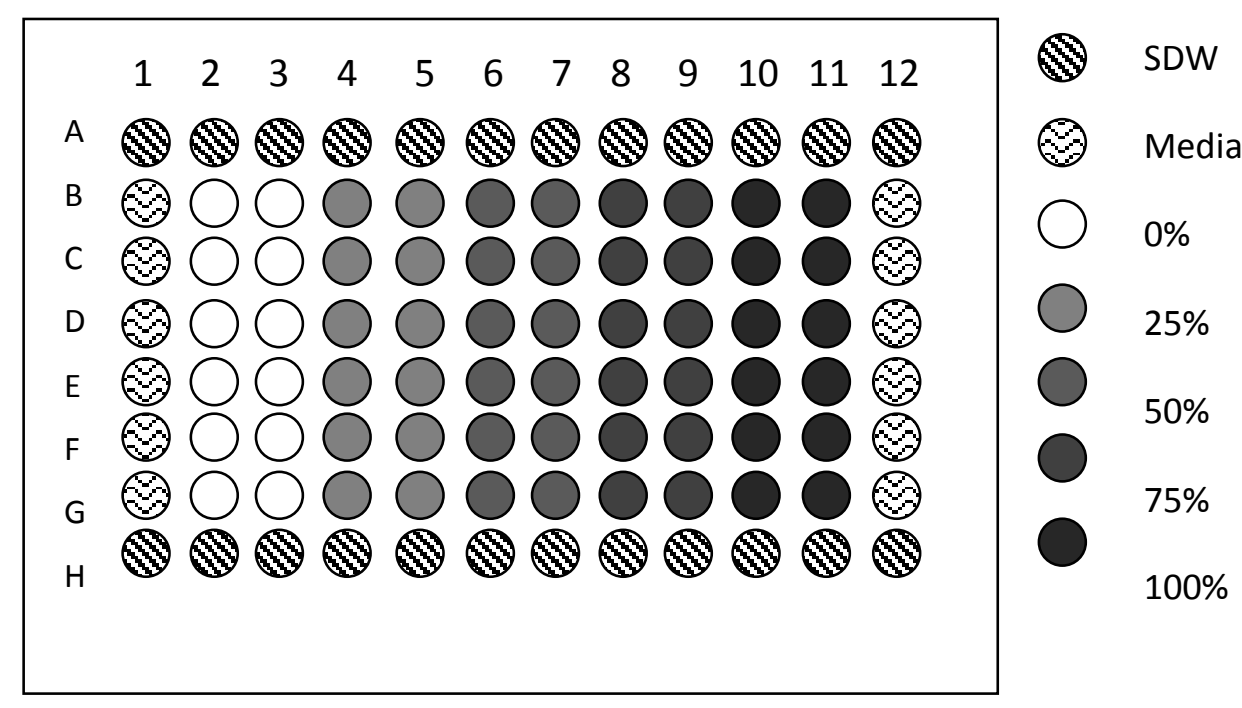

Figure 2.1: 96 wp set up for stress condition assays. Each promoter construct was tested for changes in expression in response to a range of stress factors, displayed in table 2.3. SDW and media in the outside wells prevented evaporation and provided a media control to establish contamination and background fluorescence.

\begin{tabular}{|c|c|c|c|c|c|c|}
\hline Stress & \multicolumn{5}{|c|}{ Final Concentration } & \multirow{3}{*}{$\begin{array}{l}\text { Media } \\
\text { HdeB }\end{array}$} \\
\hline & $0 \%$ & $25 \%$ & $50 \%$ & $75 \%$ & $100 \%$ & \\
\hline \multicolumn{6}{|c|}{ Chelators (mM): } & \\
\hline EDTA & 0 & 0.25 & 0.5 & 0.75 & 1 & \\
\hline EGTA & 0 & 0.25 & 0.5 & 0.75 & 1 & \\
\hline EDDA & 0 & 0.25 & 0.5 & 0.75 & 1 & \\
\hline \multicolumn{6}{|c|}{ Antibiotics $(\mu \mathrm{M})$} & LB \\
\hline SM & 0 & 0.19 & 0.39 & 0.78 & 1.56 & \\
\hline TC & 0 & 0.1 & 0.2 & 0.35 & 0.75 & \\
\hline RIF & 0 & 0.39 & 0.78 & 1.56 & 3.125 & \\
\hline INH & 0 & 3.125 & 6.25 & 12.5 & 25 & \\
\hline \multicolumn{6}{|c|}{ Nutrient Starvation (mLs see appendix 7) } & HdeB \\
\hline Carbon & 6 & 8 & 12 & 16 & 20 & \\
\hline Nitrogen & 0.1 & 2.5 & 5 & 7.5 & 10 & \\
\hline Phosphate & 0.1 & 2.5 & 5 & 7.5 & 10 & \\
\hline Others (\%) & & & & & & LB \\
\hline $\mathrm{EtOH}$ & 0 & 2 & 3 & 4 & 5 & \\
\hline SDS & 0 & 0.02 & 0.03 & 0.04 & 0.05 & \\
\hline
\end{tabular}

Table 2.3: Stress Conditions. 


\subsubsection{Nutrient starvation}

$15 \mathrm{~mL}$ overnight cultures of $M$. smegmatis containing the three TCS controlled promoters ( $p h o A, h s p X$ and MtrA) as well as the negative control pSHigh construct were grown at $37^{\circ} \mathrm{C}$ with agitation in $\mathrm{HdeB}$ minimal media. These were then diluted to an $\mathrm{OD}_{600}$ of 0.1 in a range of 5 different media starvation conditions $(0 \%, 25 \%$, $50 \%, 75 \%$ and $100 \%$ of each nutrient) and transferred to a $96 \mathrm{wp}$, sealed and incubated at $37^{\circ} \mathrm{C}$ with agitation for 48 hours. Plates were then removed and read for OD and F. A typical 96 wp set up is displayed in figure 2.2. Column 1 of the 96 wp was filled with SDW to prevent evaporation in other wells and column 12 was used for media controls.

LB was used to grow $15 \mathrm{~mL}$ overnight starter cultures, with the exception of the phosphate starvation and chelator experiments for which HdeB minimal media was used. These were then diluted to an $\mathrm{OD}_{600}$ of 0.2 , transferred to $96 \mathrm{wps}$, sealed and incubated with agitation at $37^{\circ} \mathrm{C}$ for 48 hours.

\subsubsection{Antibiotic stress}

Sub-inhibitory concentrations of 4 antibiotics were tested in this manner namely, RIF, $\mathrm{INH}, \mathrm{SM}$ and tetracycline. The concentration of each antibiotic ranged from half the minimum inhibitory concentration (MIC) of each drug in $M$. smegmatis so that the antibiotics would not inhibit growth of the cells in the assay. Table 2.5 indicates final concentrations in LB used in this experiment for each antibiotic. 


\subsubsection{Chelators}

A number of commercially available chelators from Sigma Aldrich were used in this experiment to starve the media of cations; EDTA (Ethylene Diamine Tetraacetic Acid), EGTA (Ethylene Glycol Tetraacetic Acid) and EDDA (Ethylene Diamine-N,N'Diacetic Acid ) all of which quench media of metal ions. Up to $1 \mathrm{mM}$ of each chelator was added to the media in a $96 \mathrm{wp}$ before cells were added. For final concentrations used see table 2.3.

\subsubsection{Additional Stressors}

Cells were grown to mid exponential phase in LB and then transferred to three 96 wps where they incubated for half an hour at $4{ }^{\circ} \mathrm{C}, 37^{\circ} \mathrm{C}$ and $45^{\circ} \mathrm{C}$ without shaking. Plates were then returned to $37^{\circ} \mathrm{C}$ for half an hour before being read for OD and $\mathrm{F}$. Other stress conditions include low levels of SDS ( $0-0.05 \%)$ and ethanol $(0-5 \%)$ in the LB.

\subsubsection{Validation of Expression Conditions}

Initial findings from the 96 wp assays were repeated with an increased culture volume of $50 \mathrm{~mL}$ in $250 \mathrm{~mL}$ flasks. Triplicate overnight cultures were inoculated and diluted in the same way, then incubated with agitation at $37^{\circ} \mathrm{C}$ for 48 hours before $\mathrm{OD}$ and $\mathrm{F}$ were measured. 


\subsection{Screening For TCS Inhibitors}

\subsubsection{Screening Compound Libraries}

Overnight cultures of $M$. smegmatis containing pShigh_mtrA were grown in $15 \mathrm{~mL}$ rich media with agitation and then diluted to an $\mathrm{OD}_{600}$ of 0.2 . An appropriate number of 96 wps for each library were dispensed with $50 \mu \mathrm{L}$ of rich media supplemented with $\mathrm{Kan}_{50}, 0.1 \%$ Tween $_{80}$ and D-arabinose ${ }_{100}$ in each well excluding one column of SDW to prevent evaporation, as shown in figure 2.6. Using a CyBio liquid handler, 1 $\mu \mathrm{L}$ of each compound, from a $1 \mathrm{mM}$ stock dissolved in DMSO, was dispensed to 50 $\mu \mathrm{L}$ of media giving a final concentration of $20 \mu \mathrm{M}$ of each compound in a final volume of $100 \mu \mathrm{L}$, after the addition of cells. Diluted cells were aliquotted to each well giving an optical density of 0.1 at $600 \mathrm{~nm}$. Controls were added to column 12 including cells in rich media with and without DMSO as well as two antibiotic controls usually rifampin and capreomycin to show background media fluorescence.

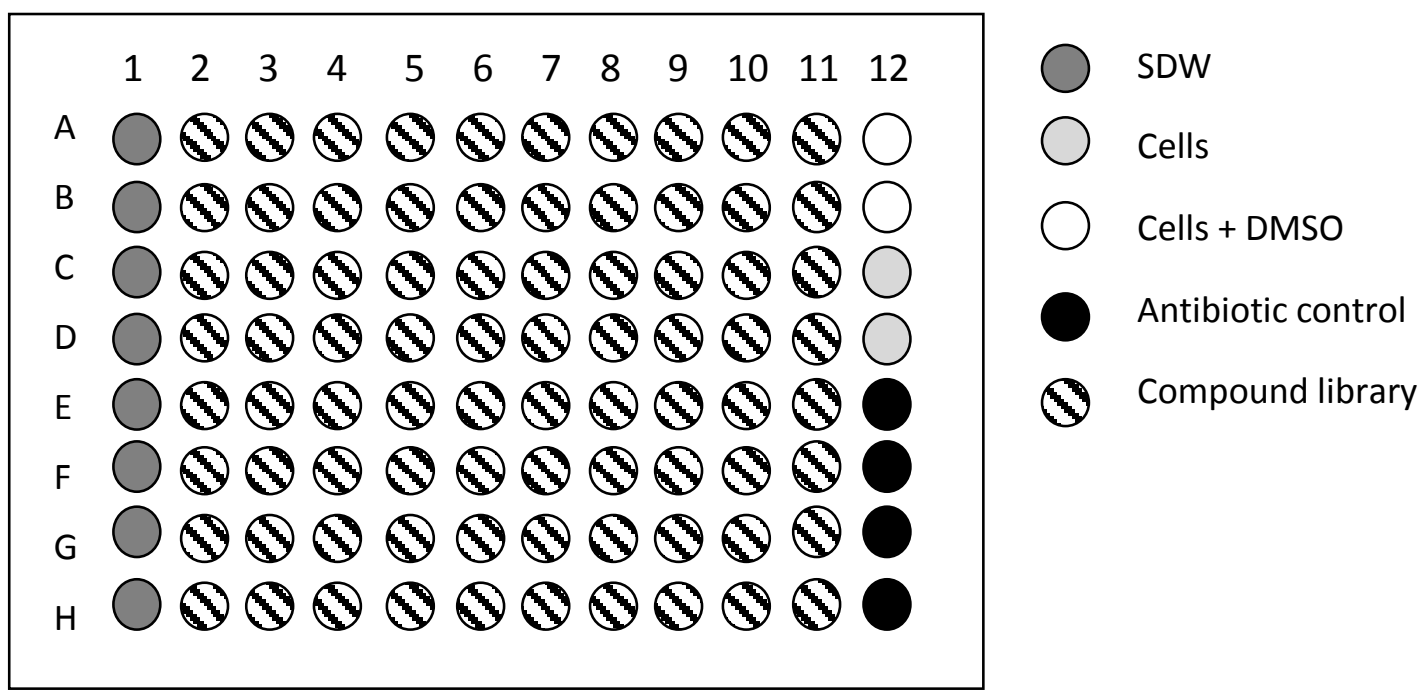

Figure 2.2: 96 well plate set up for compound library screening. Compounds were transferred by a liquid handler from stock solutions to 96 wps. Every well represents a different compound, except for the outside wells which contain water, on the left and controls on the right. 
Plates were then sealed with plate seals and parafilm and wrapped in gladwrap before incubation at $37^{\circ} \mathrm{C}$ with shaking for 96 hours. Plates were read by a Wallac Envision plate reader for $\mathrm{OD}_{580}$ and $\mathrm{F}$ (excitation $488 \mathrm{~nm}$ and emission $509 \mathrm{~nm}$ ). The phoA promoter driven expression was also tested in a similar way except that minimal media was used, supplemented with $\mathrm{Kan}_{50}$ and $0.5 \mathrm{mM}$ EDTA. For these plates an additional control of promoterless pShigh was added, instead of having 4 antibiotic controls.

\subsubsection{Palmitoleic and Oleic Acid Serial Dilutions}

Three $2 \times$ dilutions of each of the fatty acids and a solvent control of ethanol were set up in rich media starting from a final concentration of $50 \mu \mathrm{M}$. An ethanol solvent control was included and these values are shown in the results as $0 \mu \mathrm{M}$ of each type of fatty acid. Cells with an initial $\mathrm{OD}_{600}$ of 0.1 were added to this media, including strains containing the plasmids pSHigh, pSHigh_mtrA and pSHigh_hsp60. These were incubated and measured for $F$ and OD as described in HTP screen. 


\section{CHAPTER THREE: \\ Construction of a GFP reporter plasmid}




\subsection{Introduction}

In order to screen for potential TCS inhibitors, this study required a method of determining the level of TCS controlled gene expression under different stress conditions, which could be modified to a $96 \mathrm{wp}$ format for high throughput screening. The green fluorescent protein has been used for over a decade, as a reporter of gene expression ${ }^{133}$ in a number of organisms including mycobacteria ${ }^{134}$. By inserting the promoter sequence of a gene of interest in front of gfp on a plasmid, the amount of protein expressed under the control of this promoter will be indicative of promoter activation, which can be measured by the fluorescence intensity.

Since its isolation from A. victoria, GFP has been engineered to suit a number of applications across many fields in biology. A number of enhanced variants, known as eGFPs, were developed by Cormack et al in order to increase the signal strength and stability of the protein ${ }^{136}$. Taking a rational engineering approach, they used site directed mutagenesis to vary the 20 amino acids surrounding the central chromophore and screened for improved signal strength with fluorescence activated cell sorting (FACS). The result was GFPmut2, with a 100 fold increase in fluorescence compared with wild type GFP and a red shift in its natural excitation and emission spectra from $395 \mathrm{~nm}$ to $488 \mathrm{~nm}$, making it more compatible with techniques such as FACS and FITC filtration ${ }^{136}$.

In this study GFPmut2 was chosen as a reporter over other methods, such as $x y l E$ expression, for a number of reasons. Firstly, as GFP is activated by light of a specific wavelength there is no substrate required, which can interfere with other cellular processes. Secondly, the intensity of a fluorescent signal can be easily measured using a FITC filter under a microscope, and quantified in a 96 wp fluorescence 
reader. Finally, this reporter is well established as a measure of gene expression in mycobacteria ${ }^{134}$.

A number of mycobacterial promoters are constitutively expressed and induce protein formation all the time; these are sometimes known as "housekeeping" genes. GFP expression under the control of the constitutively expressed promoter from the hsp60 gene, for example, gives off relatively high levels of fluorescence. Many others, such as the TCS-controlled genes which were selected in this study, induce GFP expression under certain conditions, but never at the same level as the $h s p 60$ promoter. In order to monitor relatively small changes in gene expression as a result of stress conditions, a very sensitive reporter system was required. The GFPmut2 variant has been well adapted to measure bacterial gene expression; however another means of improving signal strength is to increase the number of plasmids per cell.

Bourn et al showed that a modification to the pAL5000 mycobacterial origin of replication on a plasmid resulted in a seven fold increase in plasmid copy number per cell ${ }^{138}$. In this chapter, the development of the new plasmid pSHigh containing gfpmut2 and the modified pAL5000, will be described. Its relative fluorescence will be established and compared with that of low copy number plasmids containing the same GFPmut2 under the control of the $h s p 60$ promoter. Having demonstrated an increase in signal strength, the $p h o A, h s p X$ and $m$ trA promoters from $M$. smegmatis were amplified and used to replace the $h s p 60$ promoter for further analysis of the TCS they control. 


\subsection{Results}

Construction of the reporter plasmid pSHigh involved a number of steps ${ }^{142}$. An overview of the process in shown in figure 3.1 

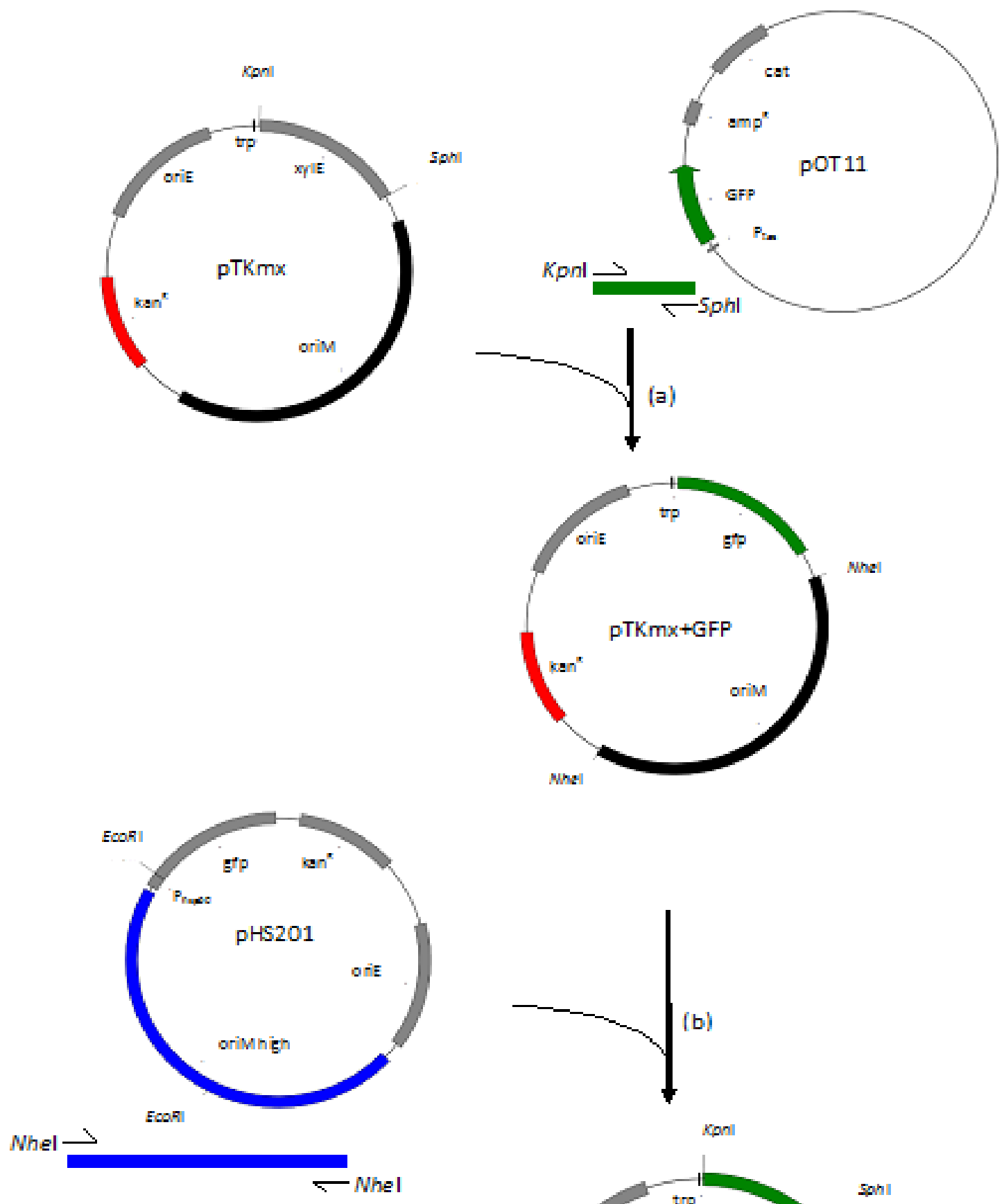

Figure 3.1. Construction of pSHigh, an optimised GFP reporter plasmid. The gfp gene, along with its ribosomal binding site (RBS), was amplified from pOT11 using GFP_RBS_F and GFP_R primers. This fragment replaced the xyIE of pTKmx using its $\mathrm{Kpn} /$ and Sph/ restriction sites to make pTKmx_GFP. The modified oriM of pHS201 was amplified with

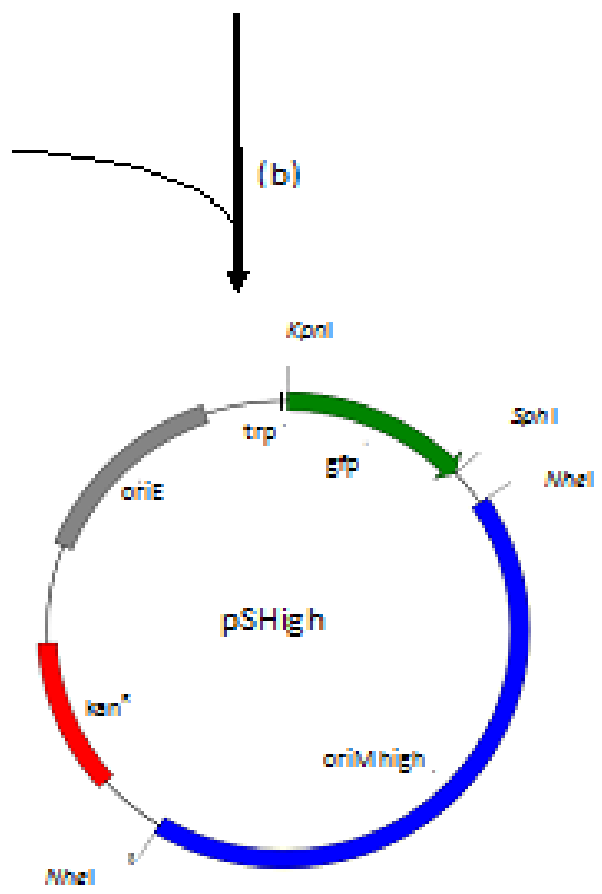
oriM_F and oriM_R primers and this modified origin of replication replaced the oriM region of pTKmx_GFP using the surrounding Nhel sites. 


\subsubsection{Amplification of GFPmut2 and OriM by PCR}

The optimised reporter plasmid pSHigh was developed by cloning a new origin of replication oriM high and gfpmut2 into a mycobacterial plasmid pTKmx created by Kenney and Churchwood ${ }^{137}$. Figure 3.2 shows the PCR products of the amplification of gfpmut2 from pOT11 and oriM high from pHS201 on $1 \%$ agarose. A gradient PCR showed that the optimal annealing temperature for cloning the oriM high fragment was $63^{\circ} \mathrm{C}$, as shown in figure 3.2 (a).

A.

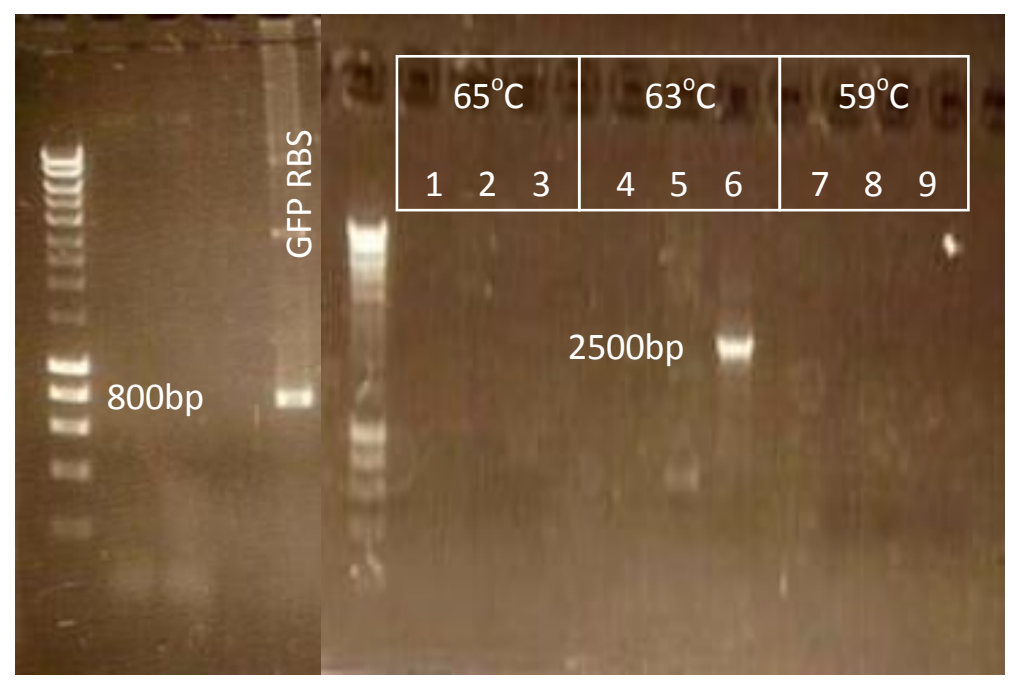

Figure 3.2: Visualisation of gfpmut2 and oriM high amplified PCR products. A) gfpmut2 along with its RBS was amplified by PCR using pOT11 as a template, giving an $800 \mathrm{bp}$ product on the agarose gel. B) OriM was amplified effectively from pHS201 using an annealing temperature of $63^{\circ} \mathrm{C}$, giving a 2500 bp product shown in the sixth lane. 


\subsubsection{Switching $x y I E$ and pAL5000 with GFPmut2 and oriM high in pTKmx}

The mycobacterial plasmid pTKmx was used as the backbone for pSHigh. Kenney et al used this plasmid to monitor promoter activity with the $x y / E$ reporter system ${ }^{137}$, which we replaced with gfpmut2. OriM high was substituted for pAL5000 to increase the copy number of the plasmid per cell. Figure 3.3 illustrates how this plasmid was cut with restriction enzymes Kpnl and Sphl to remove xylE and Nhel to remove pAL5000. The white boxes on the gel show the bands which were removed and gel purified, pTKmx - xylE and pTKmx -pAL5000.

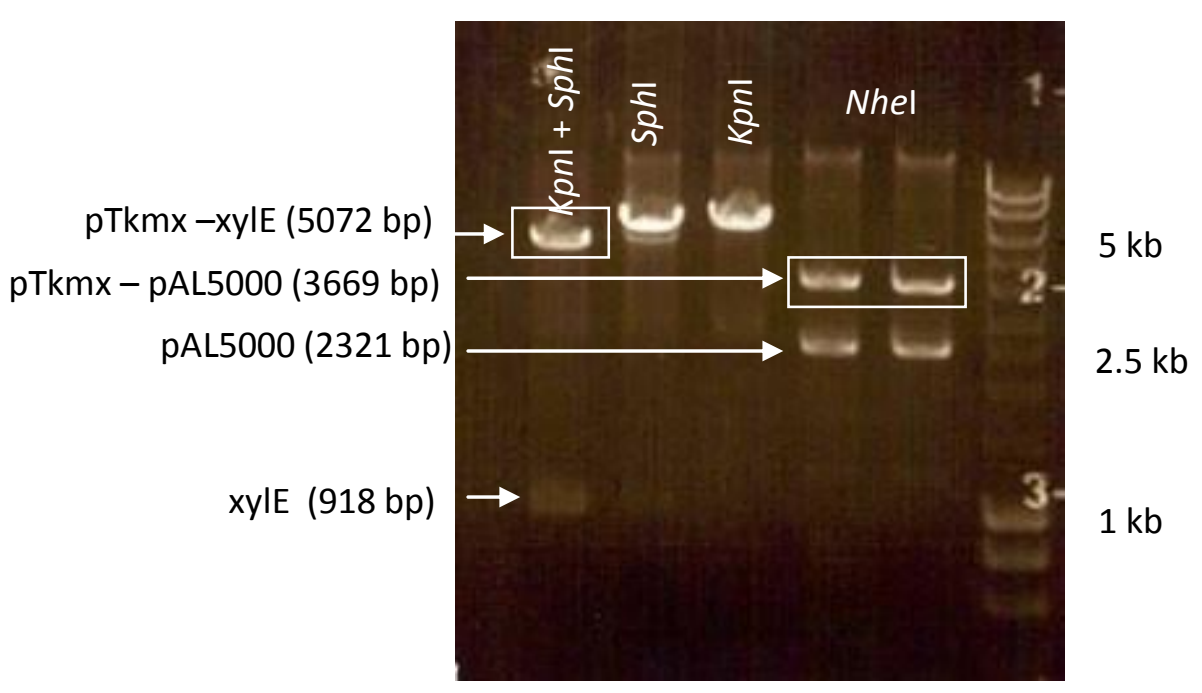

Figure 3.3: Removal of xyIE and pAL5000 from $\mathrm{pTKmx}$ by restriction enzyme digest. Kpnl and Sphl were used to remove the xylE fragment and Nhel to remove pAL5000 from pTKmx giving band sizes corresponding to the size of these fragments in relation to the plasmid These fragments were then replaced with gfpmut2 and oriM high to make pSHigh 


\subsubsection{Comparing the Fluorescence of high and low copy number plasmids in}

\section{M. smegmatis}

The fluorescence of overnight cultures of $M$. smegmatis containing high and low copy number plasmids were compared on a single cell basis, using a microscope with a FITC filter, and on a population basis, using a 96 wp fluorescence reader. In figure 3.4 M. smegmatis cells containing different plasmids are displayed along with a map of essential elements contained on each plasmid. The high copy number plasmids, pHS201 and pSHigh_hsp60 display a much higher level of fluorescence compared with pOT62, with exactly the same GFPmut2 and RBS sequence as pSHigh_Hsp60. Images of the pHIGH100 and pSHigh plasmids show little fluorescence indicating that GFP expression is a result of the hsp60 promoter induction. The pSHigh reporter constructs were used over the pHS201 and pHIGH100 plasmids because they contained a unique cloning site directly upstream of the gfp gene and the trp terminator site from pTKmx. The cloning site allowed for the insertion of other promoters upstream of the gfp gene. It was hoped that the terminator site would reduce any background fluorescence from the gfp gene. 
A
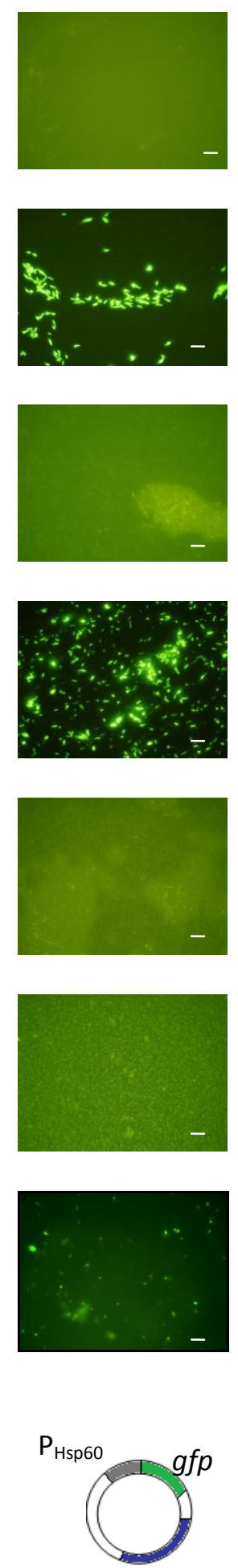

OriM (high copy number)
B
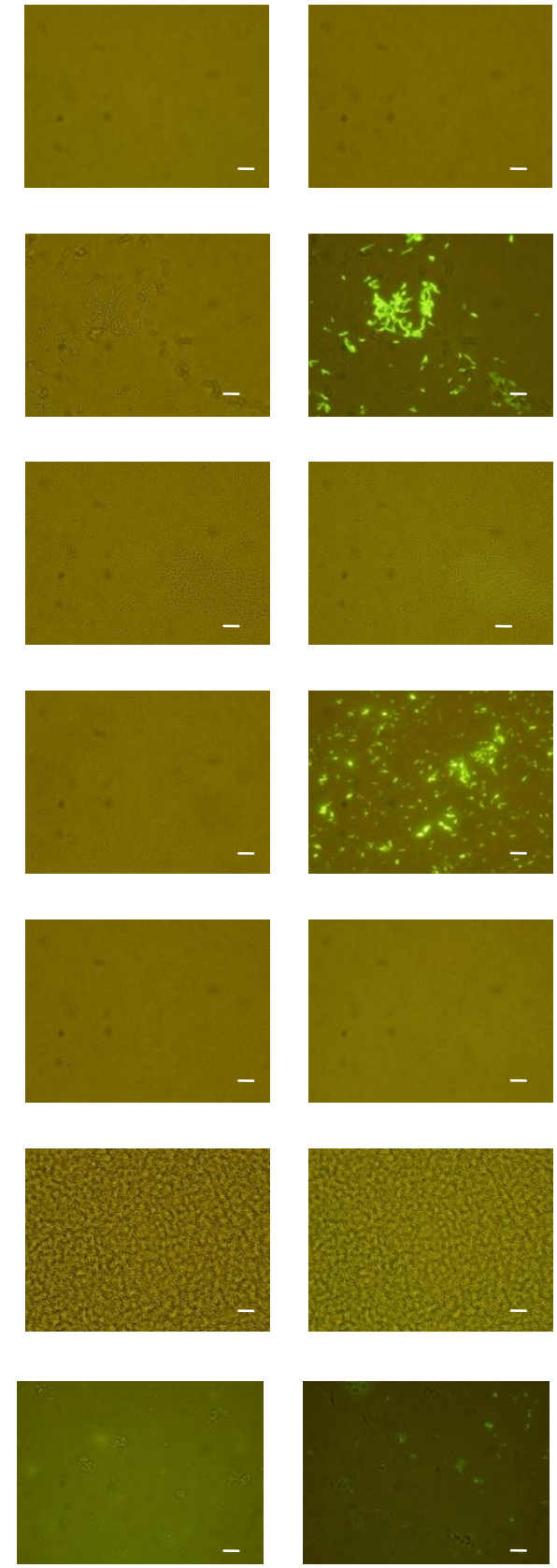
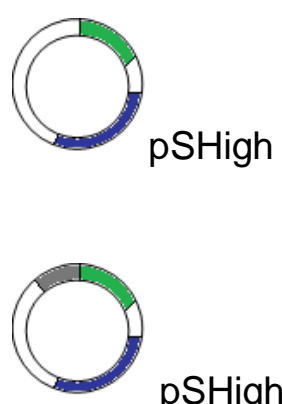

$\mathrm{pHIGH} 100$

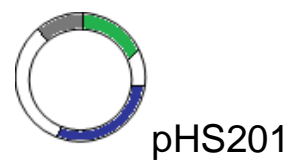

pSHigh_Hsp60

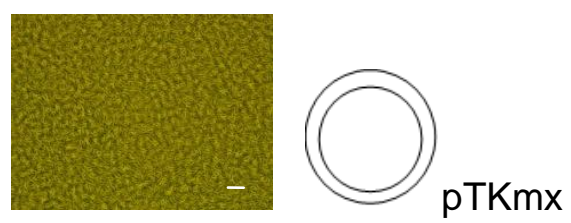

pTKmx_GFP

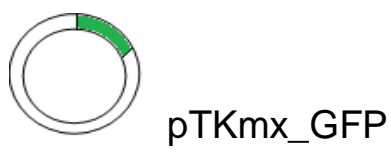

Figure 3.4: Visualisation of reporter constructs in M. smegmatis.

Cells containing plasmids used in this study were visualised for gfp expression using a blue light with a FITC filter (475 nm) (column A), white light (column B) and both (column C). Cells containing the high copy number origin of replication shown on plasmids maps in blue were significantly more fluorescence than those with the original pAL5000 origin. Low level fluorescence of the promoterless plasmids indicates no significant leakage of GFP expression. Scale bar shown in white on all photographs represents $2 \mu \mathrm{m}$. 
To explore whether the dynamic range of GFP expression was increased in a population of transformed cells we measured the fluorescence in relation to OD of the cultures. As figure 3.5 shows, the level of fluorescence in the high copy number plasmids pHS201 and pSHigh_Hsp60 is significantly higher than that of pOT62. The low level of fluorescence from pTKmx indicates that a small amount of fluorescence is picked up from cells without any GFP expression.

\section{Relative Fluorescence of Reporter Constructs in $M$. smegmatis}

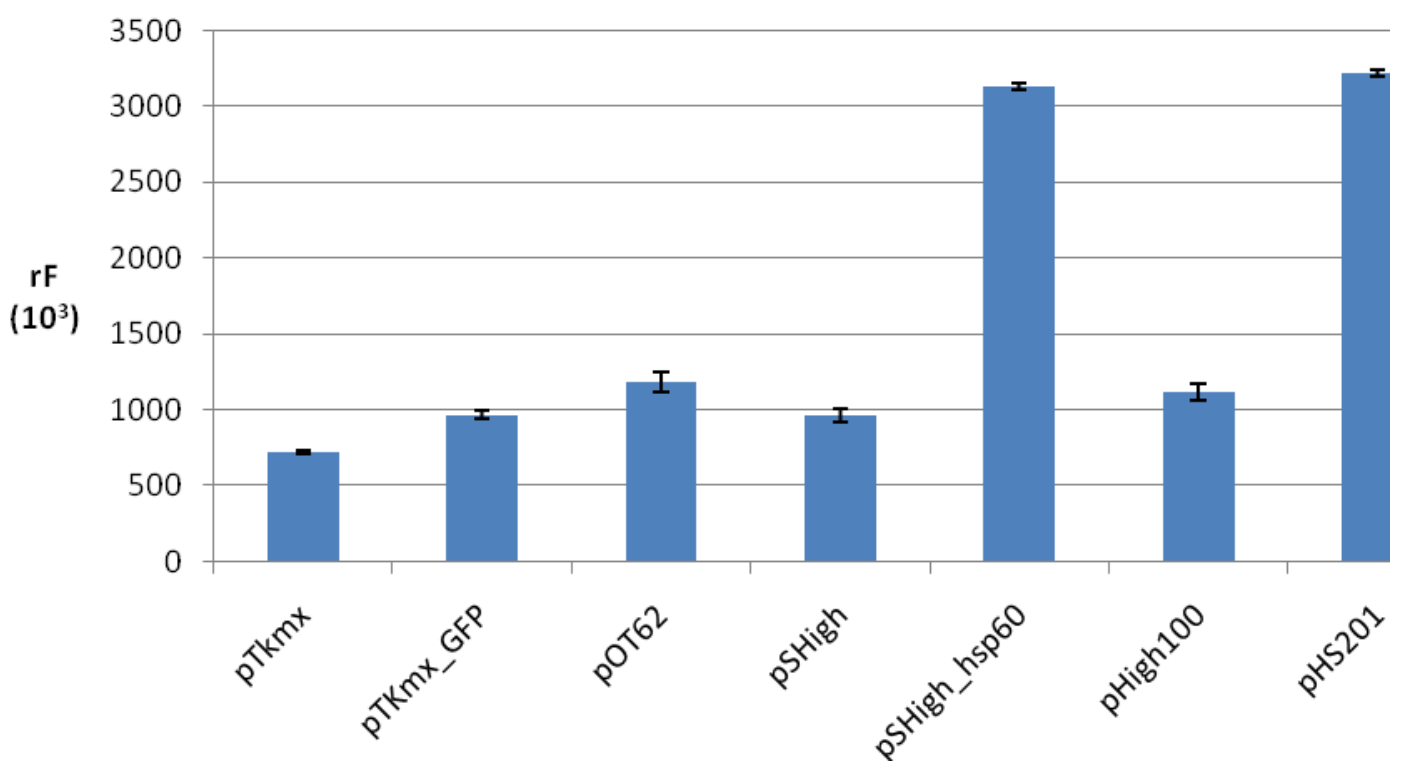

Figure 3.5: Relative Fluorescence of Reporter plasmids in M. smegmatis. High copy number plasmids pHS201 and pShigh_hsp60 display significantly higher levels of fluorescence than their promoterless counter-parts pHIGH100 and pSHigh. pOT62 containing the same promoter, RBS and gfp sequence as pSHigh_Hsp60 gives off less fluorescence, presumably as a result of the number of plasmids per cell. Error bars indicate the standard deviation between triplicate cultures.

In order to calculate the level of GFP expression the $h s p 60$ promoter conferred to a cell, the readings were normalised to show the fluorescence in relation to OD minus the background fluorescence given off by normal cells, as indicated by pTKmx. 
Table 3.1 displays the calculated normalised fluorescence and the fold induction as a result of the $h s p 60$ promoter.

\begin{tabular}{lccc}
\hline & $\begin{array}{c}\text { Normalised } \\
\text { promoterless gfp } \\
\text { expression }\end{array}$ & $\begin{array}{l}\text { Normalised } \mathbf{P}_{\mathrm{hsp60}} \\
\text { driven } g \text { fp } \\
\text { expression }\end{array}$ & $\begin{array}{c}\text { Fold } \\
\text { induction }\end{array}$ \\
\hline $\begin{array}{l}\text { Low copy number } \\
\text { pOT62, pTkmx_GFP }\end{array}$ & 245783.2 & 731867.7 & 3 \\
$\begin{array}{l}\text { High Copy number } \\
\text { pHS201, pHIGH100 }\end{array}$ & 398280.5 & 2500922 & 6.3 \\
$\begin{array}{l}\text { High Copy number } \\
\text { pSHigh_Hsp60, pSHigh }\end{array}$ & 241118.2 & 2412564 & 10.0 \\
\hline
\end{tabular}

Table 3.1: Normalised fluorescence of high and low copy number plasmids.

\subsection{Inserting TCS controlled gene promoters into pSHigh}

A literature search for TCS controlled genes in mycobacteria revealed a number of candidates. These were narrowed down to three $M$. smegmatis genes with non coding sequences upstream which could be amplified from genomic DNA and cloned into the pSHigh plasmid. A number of $M$. tuberculosis promoters were also amplified for future work in M. bovis BCG. Table 3.2 shows known TCS controlled genes from M. smegmatis and M. tuberculosis and what conditions are proposed for their induction. 


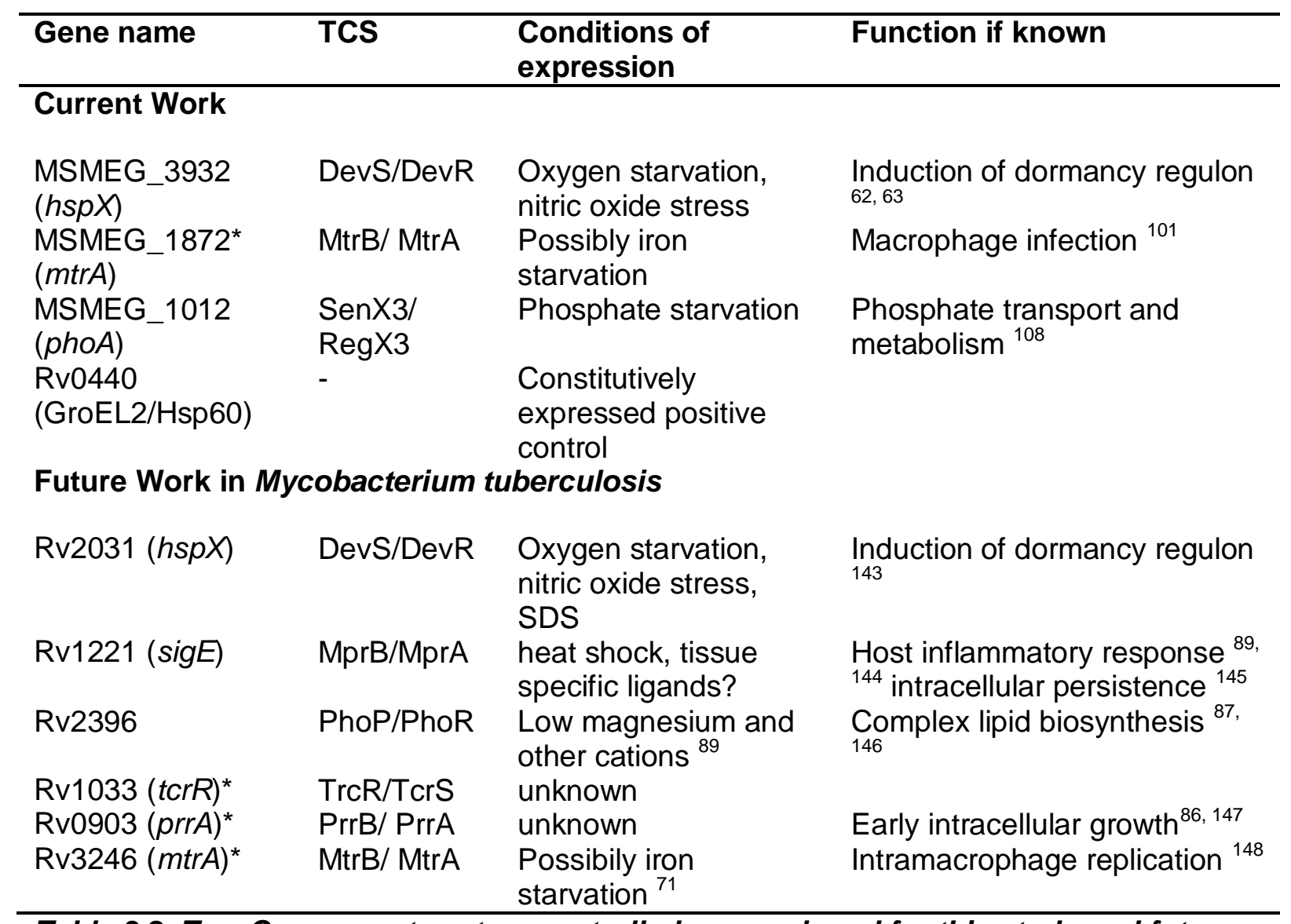

Table 3.2. Two Component system controlled genes cloned for this study and future work. ${ }^{*}$ indicates autoregulated response regulators of the TCS.

Three selected $M$. smegmatis genes, hspx, $m \operatorname{tr} A$ and $p h o A$, were used in this study.

The gene organisation and function of surrounding genes is described in figure 3.6. In order to use these genes in a promoter reporter plasmid it was important that a defined non-coding region directly upstream could be demonstrated for each. 
phoA

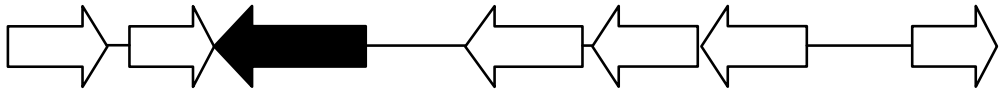

$\begin{array}{ll}\text { Gene Name } & \text { Function } \\ \text { MSMEG_1010 } & \text { Transcriptional regulator, TetR family } \\ \text { MSMEG_1011 } & \text { Short chain dehydrogenase } \\ \text { MSMEG_1012 } & \text { phoA: Alkaline phosphatase } \\ \text { MSMEG_1013 } & \text { Hypothetical protein } \\ \text { MSMEG_1014 } & \text { DNA polymerase IV } \\ \text { MSMEG_1015 } & \text { Transcriptional regulator, TetR family } \\ \text { MSMEG_1016 } & \text { Secreted protein }\end{array}$

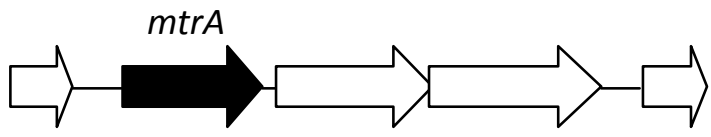

\section{Gene Name Function}

MSMEG_1873 Thymidylate kinase

MSMEG_1874 mtrA: Two component system response regulator

MSMEG_1875 mtrB: Two component system histidine kinase, OmpR family

MSMEG_1876 Lipoprotein

MSMEG_177 Hypothetical protein

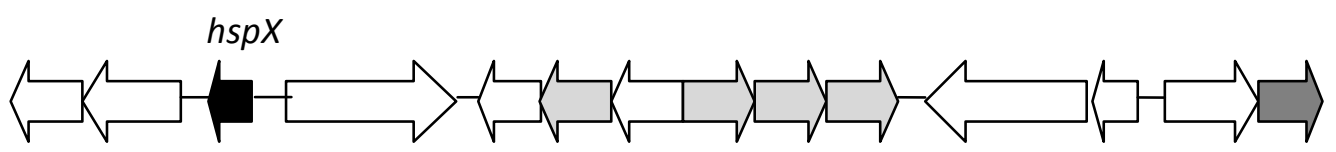

Gene Name

MSMEG_3926 to MSMEG_3931

MSMEG_3932

MSMEG_3933

MSMEG_3934, 3935 and 3937

MSMEG_3936, 3938, 3939, 3940

MSMEG_3941

MSMEG_3942

MSMEG_3943

MSMEG_3944

\section{Function}

Subunits of hydrogenase enzyme hspX homologue: $14 \mathrm{kD}$ antigen Phophophenolpyruvate synthase

Hypothetical protein

Universal stress proteins

GAF family protein

Hypothetical protein

Hypothetical protein

DevR: Two component system response regulator DevR

Figure 3.6 Gene organisation and function of selected TCS controlled genes. All selected genes possess non-coding regions directly upstream of their start codon. The genes surrounding these are named and labelled in the order that they appear from left to right. This information was downloaded from the website www.genome.jp. 


\subsubsection{Promoter Amplification by PCR from Genomic DNA}

Promoter sequences upstream of each gene were amplified from M. tuberculosis and $M$. smegmatis genomic DNA to give fragments of approximately $600 \mathrm{bp}$. Figure 3.7 shows the $1 \%$ agarose gels of the promoters amplified by PCR.

A.

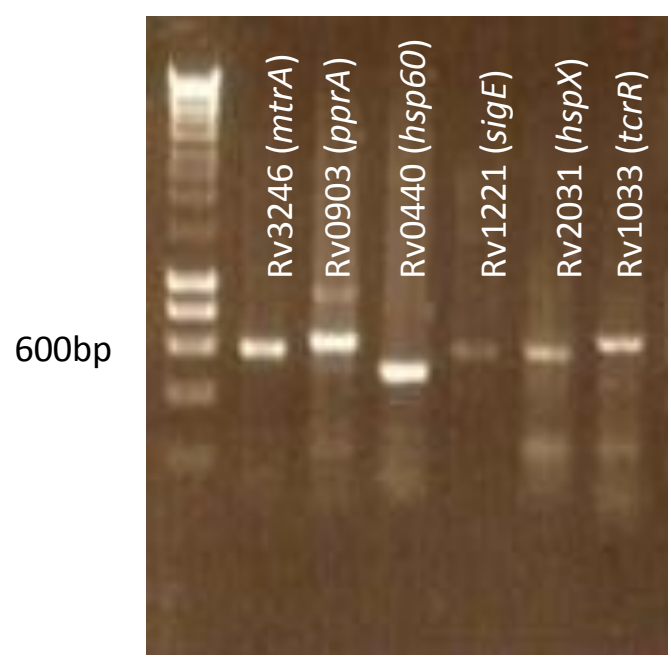

B.

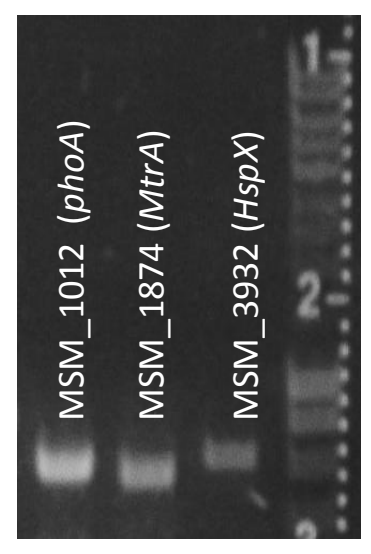

$600 \mathrm{bp}$

Figure 3.7: Promoters amplified from M. smegmatis and M. tuberculosis gDNA. A) displays five TCS controlled gene promoters of around 600bp in size, as well as the constitutively expressed hsp60 promoter amplified from M. tuberculosis genomic DNA. In B) three bands represent $M$. smegmatis promoters from selected TCS controlled genes. 


\subsubsection{Ligation of promoters into pSHigh}

In order to improve the efficiency of the ligation of promoters into the reporter plasmid, the Kpnl cut pSHigh was gel extracted to reduce the amount of uncut plasmid DNA during each ligation reaction. Figure 3.8 shows the pSHigh plasmid in its uncut state and after digestion with $\mathrm{Kpnl}$, the band removed from the gel is highlighted by a white box.

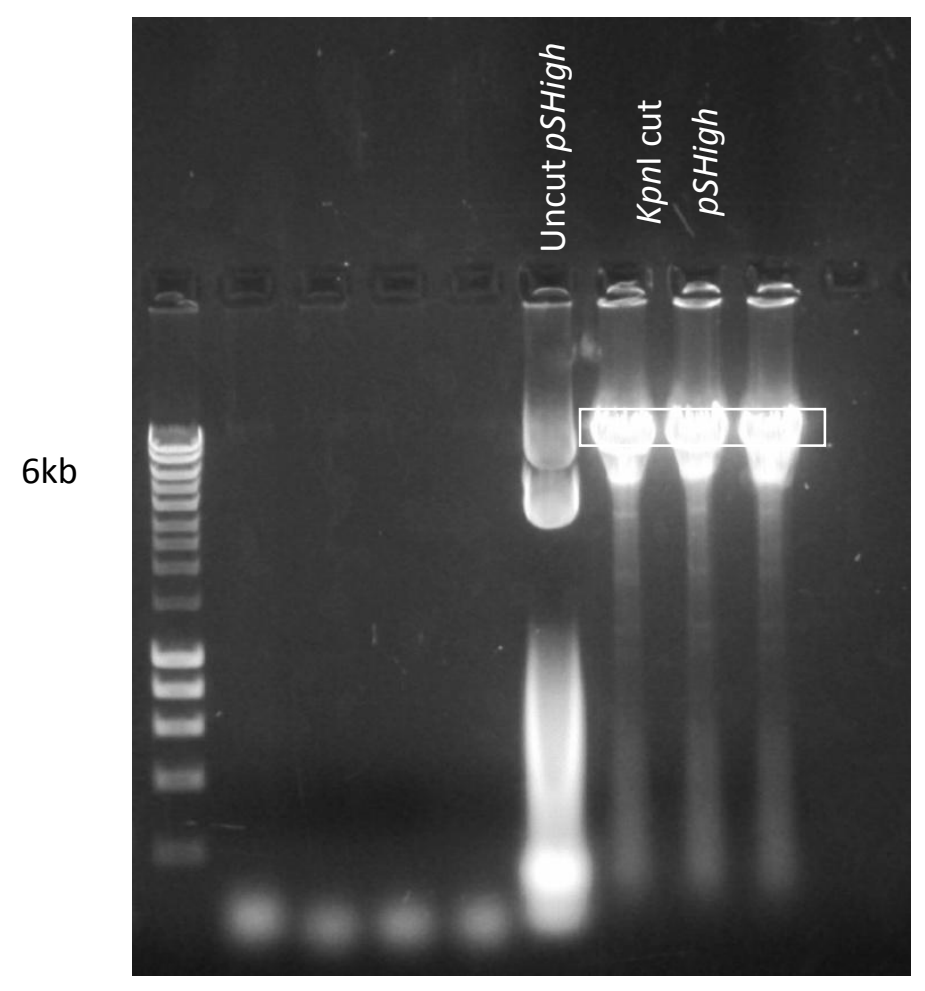

Figure 3.8: Kpnl cut and uncut pSHigh plasmid. The sixth lane shows coiled formations of the pSHigh plasmid in its uncut state. The three following lanes display pSHigh after being cut with Kpnl. The cut plasmid DNA, shown in a box, was removed by gel extraction. 


\subsection{Discussion}

\subsubsection{Optimisation of the reporter plasmid}

The principal aim of this work was to generate a cell based screen for TCS inhibitors. This required a highly sensitive reporter system which could be manipulated to identify small changes in gene expression as a result of TCS-specific conditions. The results of section 3.2.3 clearly show that pSHigh_Hsp60 is a highly fluorescent reporter construct and this is a result of the change in the origin of replication of the plasmid from pAL5000 to oriM high. Only a limited amount of background fluorescence is produced from the promoterless pSHigh plasmid, when compared with the pTKmx strain, indicating the plasmid does not express the protein independently of an adjacent promoter sequence upstream of gfpmut2. The plasmid also contains a kanamycin resistance cassette for antibiotic selection and an $E$. coli origin of replication oriE. This pSHigh_hsp60 plasmid was used as a constitutively expressed control in this study, and has also been used for screening compound libraries against mycobacteria. The plasmid displays high levels of measurable fluorescence which can also indicate cell viability when OD readings cannot be used due to interference by coloured compounds.

The authors who created the pHS201 vector describe a significant increase in plasmid copy number from around five per cell to between 32 and 64 copies of the same plasmid ${ }^{138}$. The advantage of the pSHigh plasmid over the pHS201 vector is its unique Kpnl restriction site, directly upstream from the gfpmut2 gene, allowing other promoters to be cloned in frame with the ribosomal binding site to monitor gene expression. In addition the trpA terminator sequence from pTKmx, located 
downstream of this cloning site lead to a lower level of background fluorescence from the promoterless plasmid control.

When using a reporter to monitor gene expression, a number of assumptions are made. Firstly, any changes in fluorescence demonstrated are a result of activation or repression of a particular promoter. Therefore any post translational modification to proteins in a cell which are not directly affected by activation or repression of that promoter will not be demonstrated by the reporter system. Fortunately, the three promoters selected for this work have been previously studied and RR promoter binding sites have been demonstrated. This type of method may not be suitable for other target genes if their protein concentration or activity within the cell is controlled by some mechanism other than promoter activation.

One also assumes that the number of plasmids per cell remains relatively consistent between cultures. Measuring the fluorescence of a cell indicates the amount of protein expressed therein and is dependent on the number of plasmids present. We calculate the relative fluorescence $(\mathrm{rF})$ as a measure of fluorescence divided by the OD of a culture of cells, to generate a number which is relative to the number of cells present, but we cannot account for the number of plasmids in each cell and assume that this remains relatively constant.

Another concern with this methodology is the effect a reporter may have on cellular function. The use of GFPmut2 has been established in a number of species and no significant adverse reactions to the cell have been noted. It is shown in figure 3.3 that cells expressing GFP appear normal and seem as healthy as those not expressing the protein. The benefits of this reporter over substrate based systems such as $x y I E$ are clear in this regard, although some work has indicated that 
exposure to certain wavelengths of light can cause photo bleaching of the protein, reducing its fluorescence ${ }^{149}$.

\subsubsection{Selection of TCS promoters}

As not all TCS controlled genes have had their promoters sequences published, it was important that a substantial intergenic region could be identified directly upstream of selected genes for RR binding. It is known that certain genes are controlled as part of an operon rather than direct binding at each one's promoter site, which meant some of the TCSs could not be included. Figure 3.5 indicates that genes selected for this study all qualify in this regard. We included genes such as HspX and PhoA, whose promoter binding sites are known ${ }^{108,150}$. MtrA is an autoregulated gene, in that induction of this TCS confers expression of its own response regulator ${ }^{89}$.

The importance of these TCS in terms of dormancy $(h s p X)$, phosphate starvation $(p h o A)$ and survival in the macrophage $(m t r A)$ has been discussed in some detail in the opening chapter. The conditions for $h s p X$ and $p h o A$ induction have been demonstrated in previous work, but the control of the $m$ trA gene is something of a mystery despite its proposed essentiality in M. tuberculosis. The idea of using the hspX and mtrA promoters as drug targets came from the fact that their $M$. tuberculosis homologues are involved in signal transduction mechanisms which are vital to mycobacterial survival and latency. PhoA, on the other hand is absent in $M$. tuberculosis. Its established induction during phosphate starvation in M. smegmatis and $E$. coli, however, make it an attractive target in terms of screening for a broad class of TCS inhibitors which affect a conserved part of the phosphorylation transfer and may inhibit a number of TCS mycobacteria and other species of bacteria. 


\section{CHAPTER FOUR: \\ Conditions of TCS}

Regulated Gene Expression 


\subsection{Introduction}

The basis of TCS signal transduction relies on the recognition of some environmental or internal change in conditions by the sensor kinase, leading to a congruent change in gene expression. We aimed to imitate in vivo stress conditions with a number of in vitro stressors in order to determine any changes in the expression of the TCS regulated genes.

Our assumption is that during macrophage infection and granuloma-induced dormancy the AFB is subject to considerable environmental stress; from the host immune response, nutrient starvation and a limited supply of oxygen. Although it is difficult to accurately reproduce the whole spectrum of these conditions in vitro, studies of the granuloma have suggested a number of states which can be simulated. Importantly, AFB are often found in locations within the granuloma which exhibit low oxygen tension in relevant animal models of the disease ${ }^{116}$. Despite the fact that their prominent site of infection is the lung, the AFB survive for extended periods of time in a relatively hypoxic environment.

Another key feature of the granuloma is the fact that it is made up of and constantly surrounded by host immune cells. These cells secrete chemokines, cytokines and nitric oxide products intended to rid the host of pathogenic bacilli. The host response mounted to counter a mycobacterial infection will differ from person to person, however, some of these hostile host responses can be imitated in vitro.. Heat and cold shock, changes in $\mathrm{pH}$, addition of low levels of SDS, ethanol or antibiotics to media at low levels are predicted to activate cellular stress responses which may induce the expression of TCS controlled genes involved in macrophage or granuloma survival. 
Finally, the use of HdeB minimal media can effectively control the level of particular nutrients available to cells. This media can be modified to alter the concentration of available carbon, nitrogen and phosphate. In addition to the direct removal of elements from the media, chelators can be used to remove cations such as calcium, magnesium and iron.

By measuring the effect of these stress conditions on TCS controlled gene expression we can elucidate the possible activators of these systems. As a proof of principal, $h s p X$ expression was measured during oxygen starvation, a known activator of the DevS/ DevR TCS dormancy response. This was followed by a number of other stress conditions described in the materials and methods section, which were applied to all three TCS controlled promoters phoA, mtrA and hspX. The remainder of this chapter will describe the significant findings on the influence of stress condition on TCS regulated gene expression. 


\subsection{Results}

Cells containing GFP reporter constructs were exposed to a number of stress conditions throughout this study. For simplicity, only those with a reproducible outcome will be described here.

\subsubsection{Expression of the DevS/DevR dependent $h s p X$ promoter}

The DevS/DevR TCS is known to promote the expression of $h s p X$ under conditions of oxygen starvation and nitric oxide stress ${ }^{143,151}$. Other authors have shown an increase in $h s p X$ mRNA during growth in media with $5 \%$ ethanol and to a lesser extent hydrogen peroxide ${ }^{152}$.

In order to demonstrate that the $h s p X$ reporter plasmid was oxygen dependent two oxygen starvation assays were carried out. The first was using a low copy number POT71 plasmid and a method described in a previous publication ${ }^{62}$, shown in figure 4.1. After 35 hours, the $h s p X$-driven expression increased to a level that was higher than that of the promoterless pSHigh plasmid. The positive control, hsp60 driven gene expression, is generally much higher and is variable over different time points. 
A

\section{Oxygen Starvation: $\mathrm{OD}_{600}$}

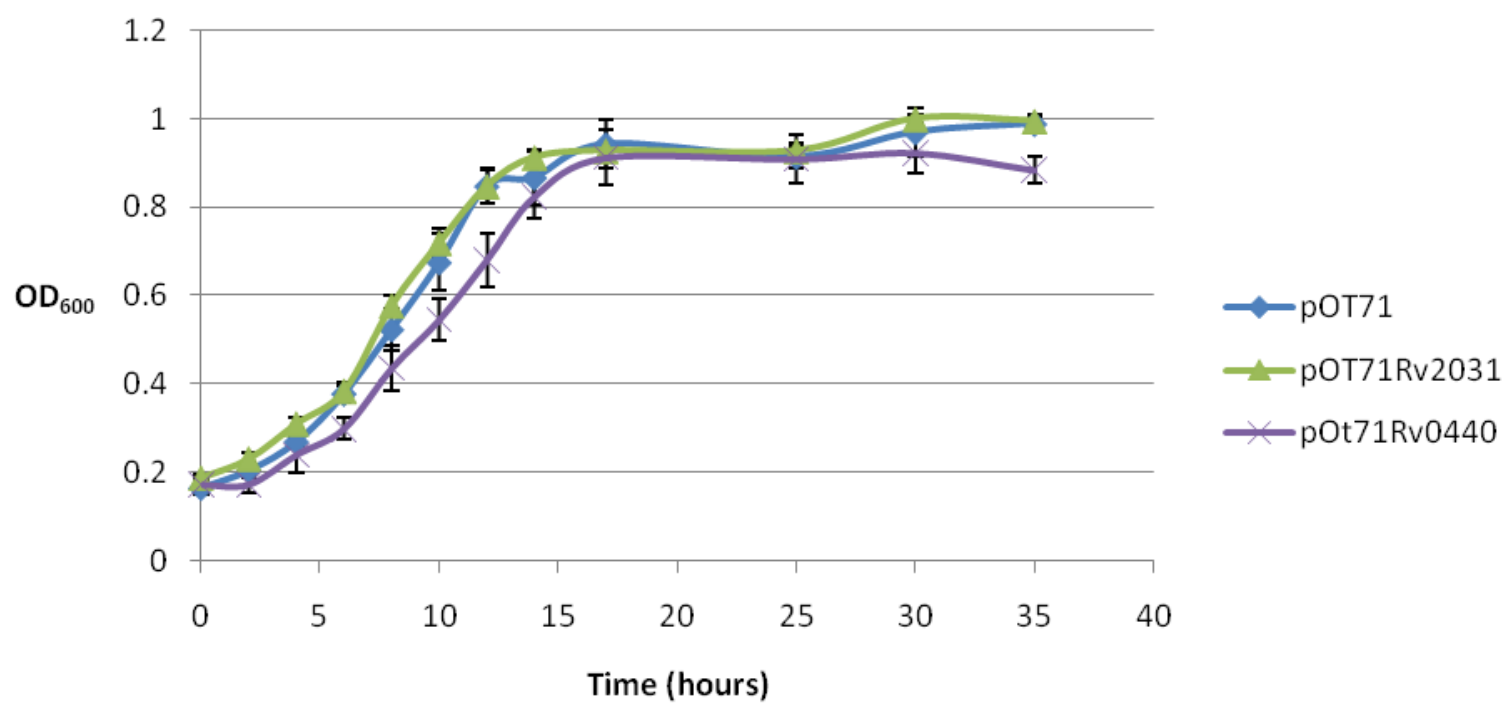

B

Oxygen Starvation: rF

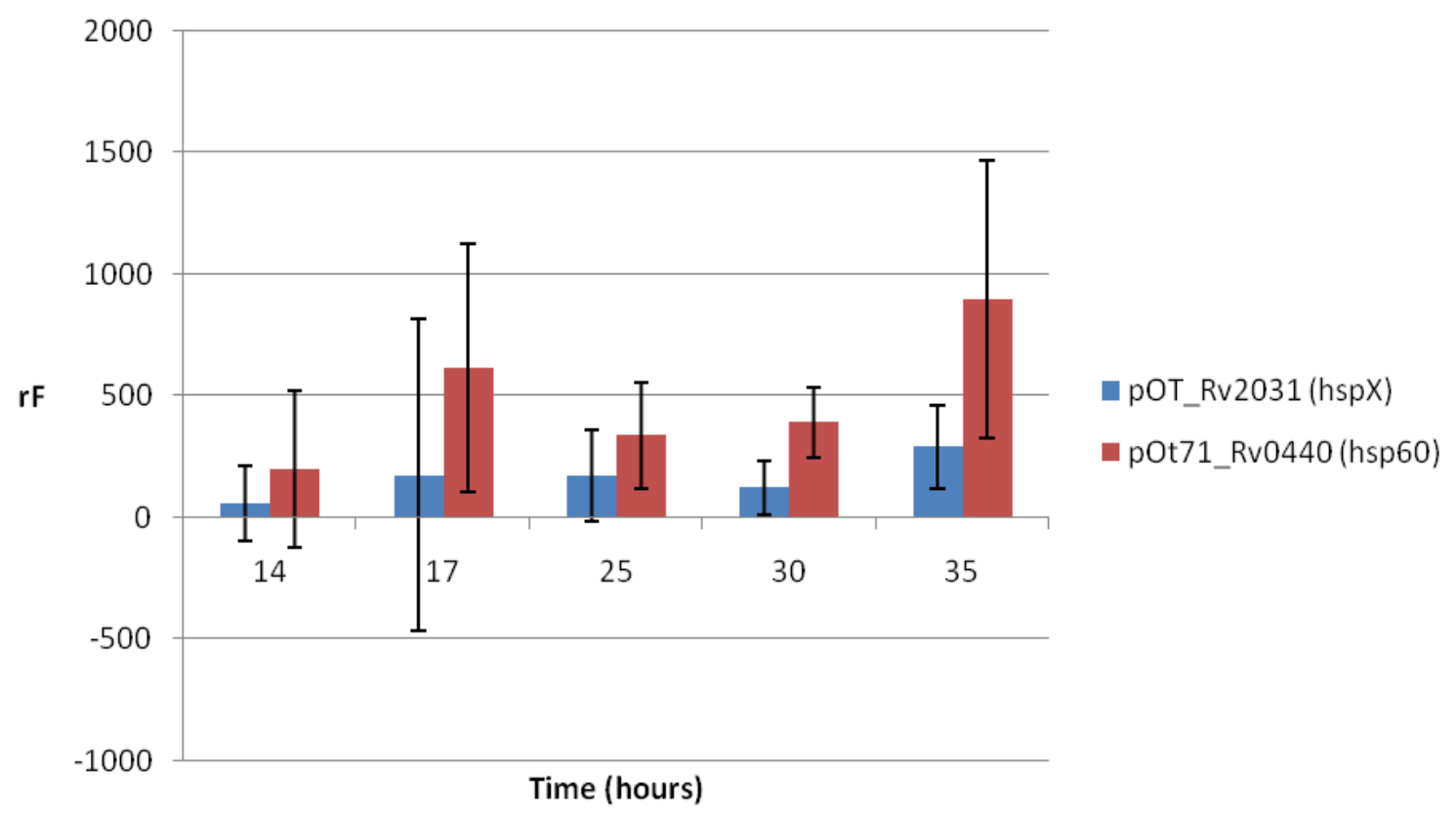

Figure 4.1: HspX expression during oxygen starvation using low copy number plasmids. In figure $A$ ) the $O D$ is shown over 35 hours of growth. When cultures reached stationary phase, at around 14 hours, cells were measured for fluorescence as shown in B). Only at 35 hours is there an increase in hspX expression. Error bars indicate the standard deviation between triplicate cultures. 
Another means of oxygen starvation was achieved based on a number of other methods used in current literature ${ }^{56,153}$. We reasoned that there were a number of problems with the original assay and made appropriate alterations to our method.

Smaller flasks with a larger volume of media were used to reduce the amount of air in each culture to start with. Another alteration to the original method was the use of rich rather than minimal media. We argued that cells are less likely to reach stationary phase as a result of some limiting factor other than oxygen, if they are living in rich media.

Using an optimised oxygen starvation method and the improved high copy number pSHigh plasmid (amongst other changes to this method described in section 2.7), a higher level of fluorescence was produced (figure 4.2). A number of changes were made to this assay to increase the stringency of oxygen starvation and observe a higher level of GFP expression under the control of the $h s p X$ promoter after 4 days. Contrasting these results with figure 4.1 indicates that a combination of the method of oxygen starvation and choice of plasmid constructs results in higher $h s p X$ promoter induction after 4 days. 


\section{Oxygen starvation: rF}

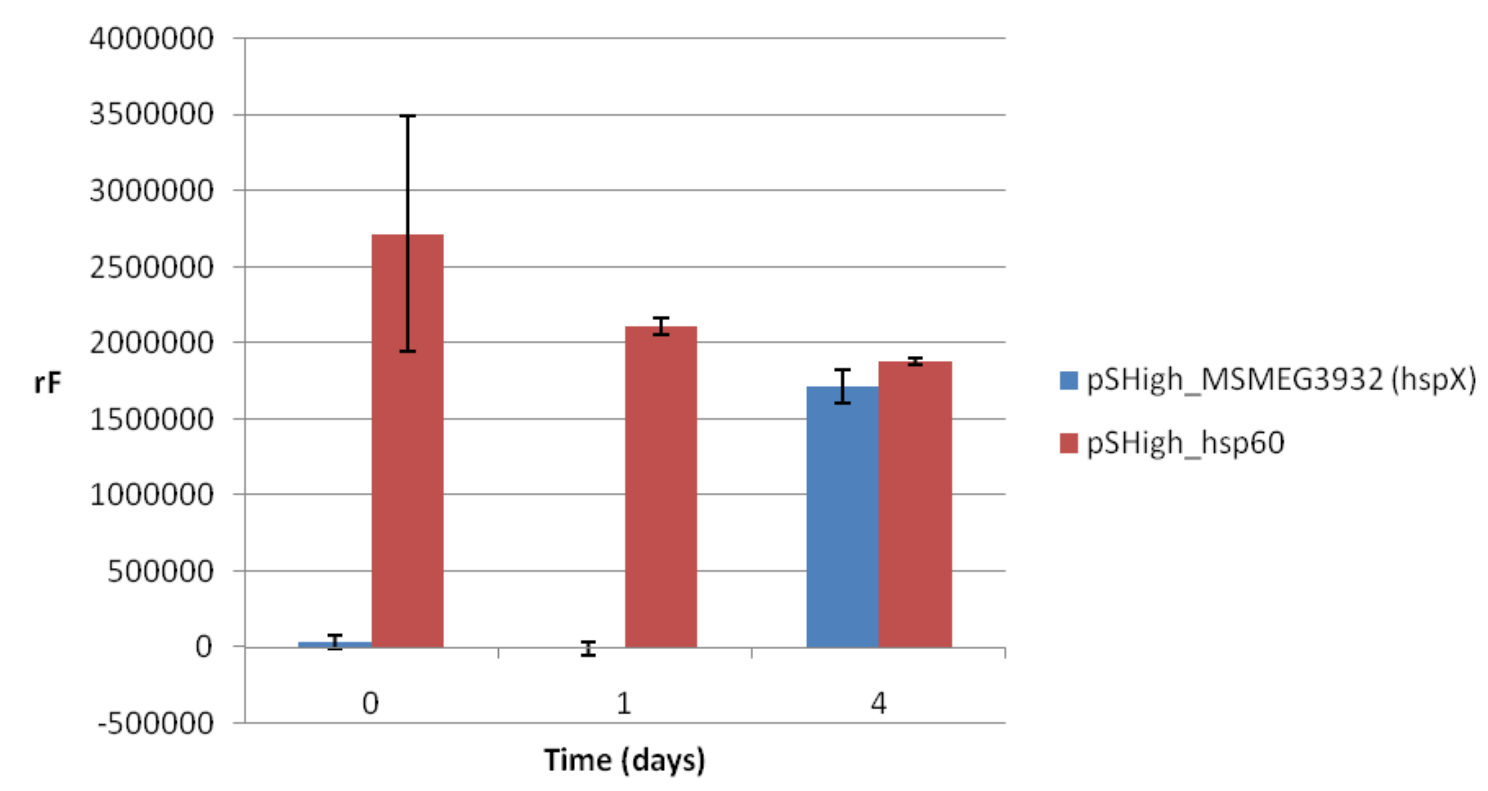

Figure 4.2: HspX expression using an optimised oxygen starvation method and high copy number plasmids. An increase in hspX expression is observed after 4 days of growth without shaking. Changes in hsp60 expression do not appear to change significantly with time. Error bars indicate the standard deviation between triplicate cultures.

\subsubsection{Expression of the SenX3/ RegX3 dependent phoA promoter}

The phoA gene of M. smegmatis is induced by the SenX3/ RegX3 TCS under conditions of phosphate starvation ${ }^{108}$. It was demonstrated that both phosphate and magnesium concentration influence the expression of the gene in our in vitro model. Using HdeB minimal media, the level of phosphate in culture media was altered to bring about stationary phase growth in a population of cells as a result of phosphate starvation. The source of phosphate in $\mathrm{HdeB}$ minimal media is a solution of $\mathrm{K}_{2} \mathrm{HPO}_{4}$ and $\mathrm{NaH}_{2} \mathrm{PO}_{4}$ added at $10 \mathrm{mM}$ and $7 \mathrm{mM}$ respectively (for more details see 
appendix 7). As figure 4.3 indicates, lower levels of phosphate in the media bring about an increase in phoA promoter activation compared with the promoterless pSHigh plasmid grown in the same condition.

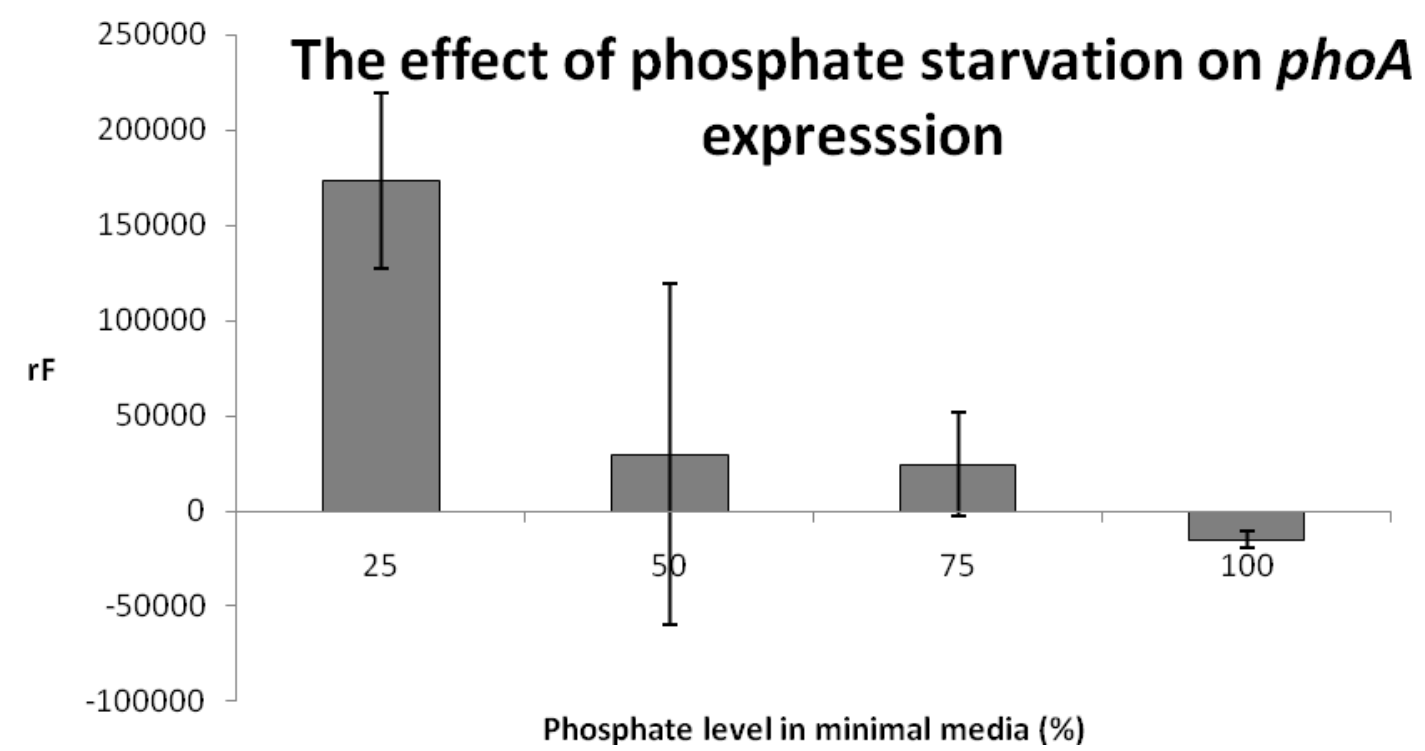

Figure 4.3: The effect of phosphate starvation on phoA expression. The amount of 100 $x$ phosphate solution added to HdeB minimal media was altered to adjust the percentage of phosphates available to cells whereby $100 \%$ is equivalent to that of minimal media. Using media with only $20 \%$ of the required phosphate leads to a change in phoA expression. Error bars indicate the standard deviation between triplicate cultures.

Figure 4.4 demonstrates that a range of EDTA concentrations also had an effect on phoA expression. A final concentration of $0.5 \mathrm{mM}$ EDTA added to minimal media seems to induce the expression of this gene. It was observed that a final concentration of more than $1.0 \mathrm{mM}$ EDTA resulted in a slow growth rate of cells, increasing the variation of the data collected, which is perhaps why fluorescence does not increase with concentration. 


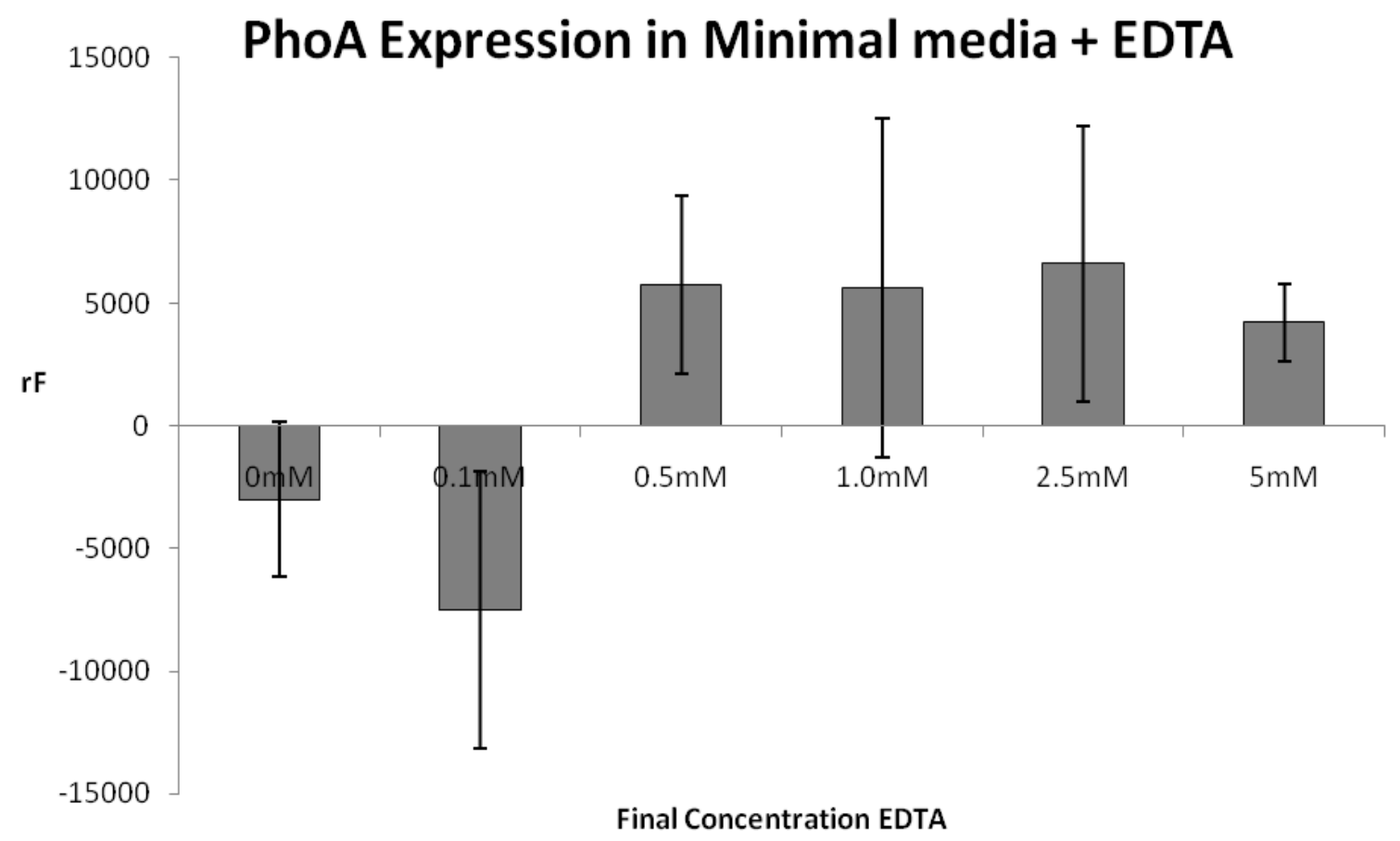

Figure 4.4: The effect of magnesium starvation on phoA expression. At a final concentration of $0.5 \mathrm{mM}$ EDTA, the fluorescence produced by the phoA promoter is increased compared with the promoterless pSHigh plasmid. Error bars indicate the standard deviation between triplicate cultures. 


\subsubsection{Expression of the MtrB/MtrA dependent $m$ trA promoter}

The GFP expression resulting from the $m$ tr $A$ promoter activation was higher than any other TCS used in this study. A higher level of fluorescence was observed in rich media and the mtrA promoter expressed GFP remained relatively consistent across all the degrees of stress that it was exposed to, with the exception of EDTA. In rich media, a control plasmid containing the reverse orientation of the promoter shows little fluorescence compared with the same sequence in the correct orientation with relation to gfp. As shown below in figure 4.5.

\section{pSHigh_mtrAin rich media}

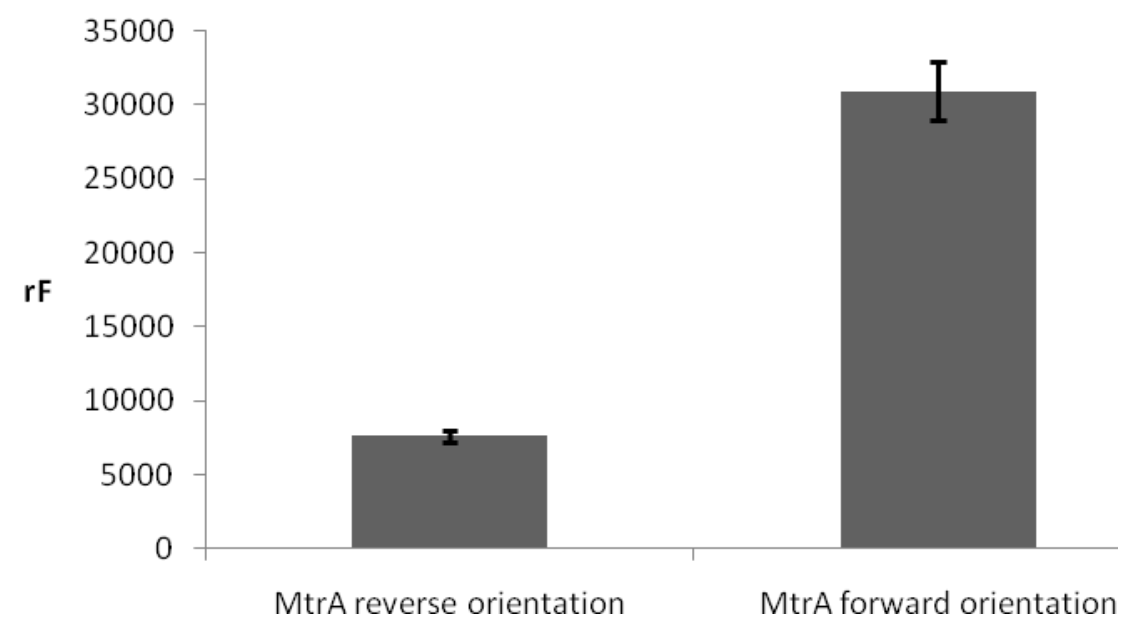

Figure 4.5: The expression of mtrA in rich media. The correct orientation of the promoter in relation the gfp on the reporter plasmid was required to give a high level of expression. Error bars indicate the standard deviation between triplicate cultures. 
There was also a change in fluorescence as a result of increasing EDTA concentration. Figure 4.6 indicates that a final concentration of $1 \mathrm{mM}$ EDTA induced a higher level of fluorescence than other concentrations. This indicates that the addition of EDTA at this concentration has an effect on the $m$ trA promoter activation, possibly due to an effect of changes to the magnesium concentration of the media.

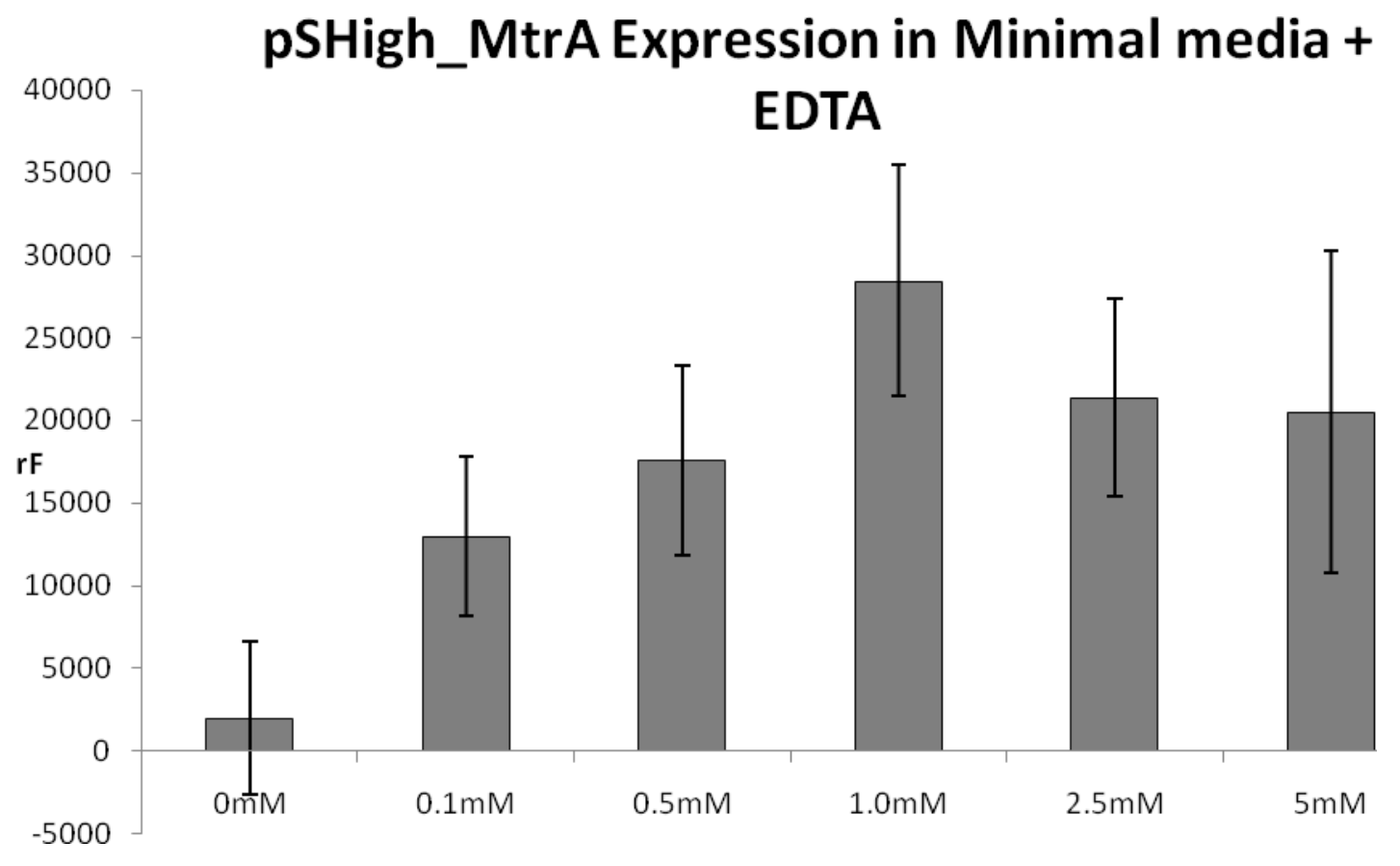

Figure 4.6: The effect of magnesium starvation on mtrA expression. A high level of expression is seen at a final concentration of $1 \mathrm{mM}$ EDTA in the HdeB media. Error bars indicate the standard deviation between triplicate cultures. 


\subsection{Discussion}

\subsection{Expression of the DevS/DevR dependent $h s p X$ promoter}

The oxygen starvation growth curves gave the highest $h s p X$-induced $\mathrm{rF}$ for any condition using this promoter construct, especially with the optimised method. As a number of variables were changed, including the time allowed for oxygen starvation, the copy number of the plasmid, the media used and volume of air in the flask to begin with, we cannot specify exactly what caused the improved results. None the less, figure 4.2 shows that $h s p X$ was induced by oxygen starvation. This provided a proof of principle that using a promoter reporter system to monitor gene expression of a TCS-controlled gene accurately.

Surprisingly, other reported activators of expression such as, SDS and ethanol stress did not give a reproducible result for this assay. There are a number of possible reasons for this, mainly relating to differences in the method used. The up regulation of $h s p X$ found by Kendall et al ${ }^{152}$ was shown by rtPCR. This method may be more effective at recognising small changes in gene expression which are a consequence of downstream effects of the stressors. Studies suggests that the sensor kinase DevS contains heme binding GAF domains, and these are likely to respond to oxygen tension ${ }^{60}$. Other authors suggest that carbon monoxide is the critical ligand of this sensor kinase ${ }^{154,155}$. The response seen to nitric oxide may result from its ability to inhibit respiration rather than direct binding ligand binding ${ }^{156}$. Similarly, ethanol and SDS may be detrimental to cells in a way that reduces aerobic respiration and induces a dormancy response. 
Another possibility is that these alternate inducers of the $h s p X$ gene occur as an effect of another stress response, rather than the hypoxia induced dormancy regulon 151. As mentioned in the introduction, the highest level of cross talk between TCS signal transduction mechanisms is the overlap in genes under their control ${ }^{90}$. HspX may be induced by a number of other signal transduction mechanisms controlled by other stressors, at a lower level than that seen during oxygen starvation.

One problem involved in this type of assay is the effect stressful conditions have on the OD of a cell culture. Adding stressors like ethanol and SDS, even at a low concentration will limit the cell growth in a solution. A small difference in concentration of a stress inducing agent between wells of a 96 wp will equate to a large difference in cell density and a high degree of variability. The only quantifiable way of showing a change is to compare the difference in fluorescence between pShigh_hspX and pSHigh, with and without that stress condition. Herein lies another problem in that any differences in growth rate between the strains used in the experiment will mask a significant result. There are several reasons that this may occur, such as how long the strain has been stored at $-80^{\circ} \mathrm{C}$, the copy number of the plasmid (in other words how much of the antibiotic resistance gene is expressed) and the burden of expressed protein on a cell.

A more relative and labour intensive method would have been to use FACS to determine fluorescence of each cell rather than an entire large culture, when using conditions which impaired growth. However relating this to a HTS assay would be near impossible. 


\subsubsection{Expression of the SenX3/ RegX3 dependent phoA promoter}

The activation of the SenX3/RegX3 controlled phoA promoter was demonstrated in two conditions; phosphate starvation and magnesium depletion. The former condition was anticipated based on $\mathrm{f}$ what is known about phoA's role in phosphate metabolism; however the result from adding magnesium chelator EDTA was not. There are a number of theories as to why this may be the case, none of which we can validate from this experiment alone.

Firstly, EDTA is a major chelator of magnesium, but it also chelates a number of other cations in media including calcium. To conclude that this effect is a direct result of magnesium starvation is an over-simplification. One could conclusively show this by setting up a minimal media depleted of magnesium and compare and contrast the result.

Another theory is that EDTA may have some effect on the cell wall integrity of the mycobacteria, or the phosphate importer/exporter pump system which is affecting the expression of this gene in some way. It was suggested by Ryndak et al that phoP mutants were unable to survive in EDTA supplemented media as a result of detrimental changes in the cell wall caused by this chelator, which could also explain what is happening in this case ${ }^{157}$.

The level of EDTA in media can have an effect on cell density, indicating it presents a certain amount of stress on a cell. It could be that this gene is upregulated as a more globally controlled stress response by the cell in addition to the SenX3/RegX3 phosphate specific induction. Another signalling system may well control the expression of this gene. Once again, the possibility of overlap in genes expressed by isolated signal transduction systems may be worth considering here ${ }^{90}$. The PhoP/ 
PhoR system is thought to be controlled by magnesium concentration and is involved in cell wall maintenance, phoA or SenX3/RegX 3 could easily be influenced by this TCS as well as the PST PIT phosphate transport systems.

It is also plausible that this TCS is controlled by more than just phosphate starvation via multiple ligand binding sites. The RegX3 sensor kinase is not itself membrane bound, interacting with a phosphate specific transporter (PST) which senses the external phosphate concentration ${ }^{108}$. Presumably, it is the result of a change in this transporter which causes dimerization of the sensor kinase and not a direct response to a reduction in phosphate acting on the kinase itself. So any other ligand affecting this phosphate transporter, the SenX3 sensor kinase or the interaction between the two may have downstream effects on the expression of phoA. A protein phosphatase assay using only the isolated HK and RR of SenX3/ RegX3 would be one way to show if the histidine kinase responded to magnesium independently of the PST.

\subsubsection{Expression of the MtrB/ MtrA dependent $m t r A$}

The activation of the $m t r A$ promoter was not higher in any of the stress conditions than in rich media. Since this gene is thought to be essential to M. tuberculosis ${ }^{101}$, it may also be essential to M. smegmatis. By definition an essential gene is one required for cell growth; therefore some constitutive expression would be expected in rich media.

Studies have shown that this gene is induced during intra-macrophage growth. Only one of the in vitro models of the macrophage or granuloma environment presented here induced an effect on mtrA expression; EDTA. Activation of $m$ trA was generally 
much lower in minimal media than that observed in rich media. As figure 4.6 shows, there is very little fluorescence without the addition of EDTA in minimal media. While this data indicates that EDTA-dependent ion depletion may have an effect on mtrA activation, this does not conclusively demonstrate that the sensor kinase directly responds to magnesium.

The inducer of this TCS is still unclear ${ }^{112,158}$ but one suggestion is iron starvation ${ }^{71}$. Rodrigues et al, who profiled iron-responsive gene expression using microarrays, saw an increase in the expression of $m$ tr $A$ during iron starvation ${ }^{71}$. In the current study, however, the use of an iron chelator EDDA did not seem to render any increase in $m t r A$ expression. This would be worth exploring, by omitting iron from the minimal media rather than using a chelator in case the EDDA concentration used in this study was not optimal and did not chelate all the iron in solution. In order to show that magnesium or iron is a ligand of the MtrB histidine kinase, further work on the crystal structure and binding properties of this protein is required. EDTA was used in this assay to deplete free magnesium from the media, but the possibility exists that it causes a multitude of effects on cellular morphology independent of specific ion starvation.

The reporter protein GFPmut2 is relatively stable; consequently endpoint readings in such an assay will not disclose exactly when the gene is induced. If $m$ tr $A$ is expressed at a particular point of the growth phase our assay picks up the GFP expression at the end of the growth assay. Using an unstable variant of GFP, such as that developed by Blokpoel et al. 2003, may give a better idea of phase dependent expression of this gene ${ }^{134}$. 
Our aim of finding a high level of GFP expression for each TCS controlled promoter was fulfilled with rich media for this TCS. Even though EDTA induced a high level of expression, its use in high throughput assays was not advantageous as minimal media gives a much higher level of variance in a $96 \mathrm{wp}$ format.

Another concern was the addition of EDTA to media as this may have a profound effect on the action of compounds used in library screens. In the end it was decided that rich media could be used for high throughput (HTP) screening because a more stable rate of growth could be achieved. Another benefit was that this data could be compared with other screens using the $h s p 60$ promoter which were also carried out in rich media. 


\section{CHAPTER FIVE:}

\section{Screening Compound}

Libraries for TCS Inhibitors 


\subsection{Introduction}

The overall aim of this project was to develop a GFP reporter and optimize conditions under which TCS controlled genes were highly expressedto develop an assay which identified compounds which inhibit this sort of signal transduction. In order to test as many compounds as possible, a high throughput method was employed, whereby thousands of compounds could be tested for their effect on the expression of these genes. Using a liquid handler, a large number of compounds could be added to separate wells of a 96 wp and incubated with $M$. smegmatis cells to observe changes in fluorescence. In theory a compound affecting any of the steps in the phospho transfer from a histidine kinase to response regulator and consequential DNA binding could cause a reduction in fluorescence driven by the TCS controlled promoter.

There are numerous benefits of using this whole cell approach to target TCS compared with isolated protein methods, as mentioned in the introductory chapter. The main advantage is that a compound inhibiting any reaction in the signal transduction mechanism may be identified, rather than targeting a single protein. Compounds which inhibit another target, resulting in a decrease in cell growth, will also display a decrease in fluorescence. By observing the OD of each well we are able to identify general cell growth inhibitors and eliminate these from the list of positive findings.

Another important point is the fact that all known TCSi affect the cell membrane in addition to any signal transduction inhibitory action. Using this screen, the OD of the cell suspension is taken into account so compounds which act on the cell in some 
other fashion, such as a detrimental effects on membrane integrity, will not be picked up.

A number of compound libraries available were tested for an effect on the activation of the mtrA promoter, including the LOPAC, Spectrum Collection, NIH diversity collections and a library of natural compounds isolated from marine organisms which will be known as the NIWA collection. MtrB/ MtrA was selected as it showed high levels of expression in rich media without the addition of growth inhibitors such as EDTA. This meant that we could expect a uniform growth rate and limited interaction between compounds and the chelator. One compound library, the LOPAC library, was screened against phoA driven expression, but as the results will show, the difference between phoA-induced expression and promoterless expression of GFP from pSHigh was too variable in this high throughput format to discern differences in fluorescence as a result of a compound interaction with the SenX3/ RegX3 TCS. The oxygen starvation assay, which was described in chapter 4 , did not translate well to a 96 wp format. It was not possible to obtain a high level of fluorescence from the $h s p X$ promoter that was uniform across the plate by increasing the volume of liquid in each well and creating an airtight seal.

In order to ascertain that there was no inhibition from the solvent used to dissolve the compound libraries, a negative control was included containing the same volume of DMSO added to two wells of each plate instead of a compound. As a positive control, to show the effect of compounds which inhibit cell growth outright, antibiotics were added to two wells of each plate. 


\subsection{Results}

\subsubsection{Compound Library Screens against $m$ trA expression}

\subsubsection{Spectrum Library against $m$ trA expression}

Fluorescence $(F)$ and OD data were plotted on a scatter graph to reveal any changes in $\mathrm{F}$ without a change in $\mathrm{OD}$, shown in figure 5.1. If a compound inhibits growth of the cells it will also lead to a decrease in $\mathrm{F}$ due to the reduction in cell number per well so we were looking for compounds which act on $\mathrm{F}$ alone. For each library, the median Fand OD readings are shown as the $\mathrm{X}$ and $\mathrm{Y}$ axes respectively so that as a rough guide compounds in the top left quarter of each graph could be considered to have an effect on promoter driven GFP expression levels. It was hoped that positive control values, displaying no effect on OD or F, would be presented in the top right quarter and negative controls, affecting OD and F would be observed in the bottom left corner.

Figure 5.1 shows a high level of variance in $F$, indicating that a number of compounds may affect either cell density or mtrA expression. In order to select only those with an effect on mtrA expression, ten outliers from the group of points were manually selected for re-testing. These are displayed by a circle on the graph and named in table 5.1.

There is a large group of compounds centred on the median mark for both $\mathrm{F}$ and $\mathrm{OD}$. They display a lower level of both $\mathrm{F}$ and $\mathrm{OD}$ than the positive control values in the top right. 


\section{Spectrum Screen with pShigh_MtrA}

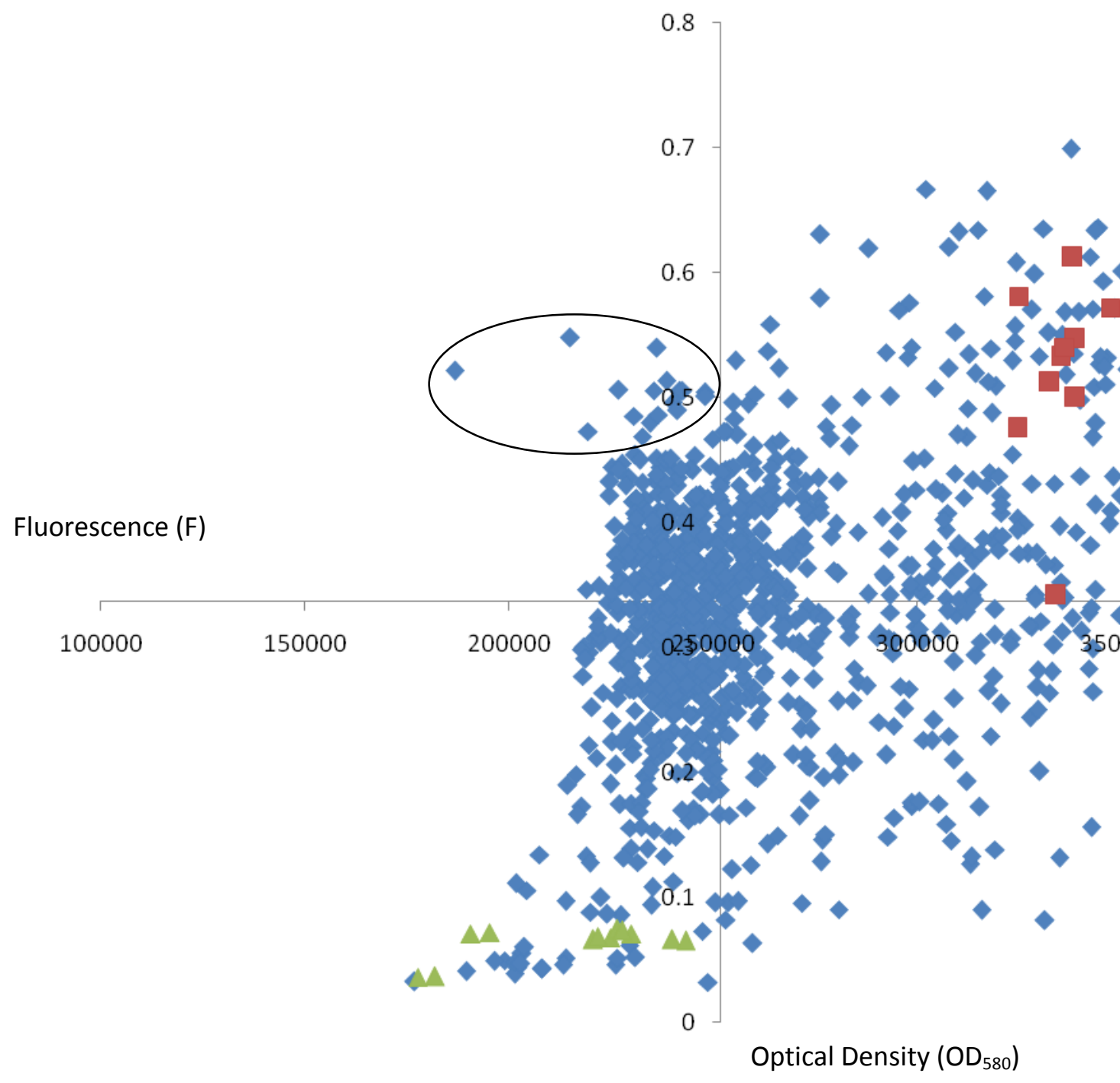

- Spectrum

upositive control $\triangle$ Antibiotic control

\section{Figure 5.1 Spectrum Screen against mtrA expression.}

The optical density and fluorescence readings are displayed for each compound in the Spectrum library. The median values have been used as axis point to divide the graph into possible inhibitors of mtrA expression which do not affect cell density, top left, and inhibitors of cell growth, bottom left. Data points which were separate from the main group were selected (circled) and re-tested. These are names in table 5.1 
Because the mtrA gene target is fairly specific, we assume that not all of these compounds are affecting the TCS. It is more likely that they affect cell growth in some other way giving a lower reading of $F$. The values circled stand out from the main group in that they do not display a lower OD than the positive control values, but display a lower $\mathrm{F}$ and are not part of the large group of compounds in the median range. The names, formulae and sources of these compounds are displayed in table 5.1 , none of which are known to be signal transduction inhibitors.

\begin{tabular}{|c|c|c|c|c|c|}
\hline NAME & Formula & Bioactivity & Source & $\mathbf{F}$ & $\mathrm{OD}_{580}$ \\
\hline STIGMASTEROL & $\mathrm{C} 29 \mathrm{H} 48 \mathrm{O}$ & \multirow{3}{*}{$\begin{array}{l}\text { cognition } \\
\text { enhancer, }\end{array}$} & $\begin{array}{l}\text { soya and calabar } \\
\text { beans; }\end{array}$ & 186735 & 0.521 \\
\hline CITICOLINE & $\begin{array}{l}\mathrm{C} 14 \mathrm{H} 26 \mathrm{~N} 4 \mathrm{O} \\
11 \mathrm{P} 2\end{array}$ & & $\begin{array}{l}\text { cytidine-5'- } \\
\text { diphosphocholine }\end{array}$ & 214927 & 0.547 \\
\hline CARNOSIC ACID & $\mathrm{C} 20 \mathrm{H} 28 \mathrm{O} 4$ & & $\begin{array}{l}\text { Salvia spp, } \\
\text { Rosmarinus } \\
\text { officinalis }\end{array}$ & 219330 & 0.472 \\
\hline $\begin{array}{l}\text { TETRAHYDRO } \\
\text { GAMBOGIC ACID }\end{array}$ & $\mathrm{C} 38 \mathrm{H} 48 \mathrm{O} 8$ & & derivative & 226869 & 0.506 \\
\hline PRISTIMEROL & $\mathrm{C} 30 \mathrm{H} 42 \mathrm{O} 4$ & & $\begin{array}{l}\text { derivative of } \\
\text { pristimerin }\end{array}$ & 230552 & 0.485 \\
\hline $\begin{array}{l}\text { 7-OXO } \\
\text { CHOLESTERYL } \\
\text { ACETATE }\end{array}$ & $\mathrm{C} 29 \mathrm{H} 46 \mathrm{O} 3$ & & Cliona copiosa & 232658 & 0.469 \\
\hline $\begin{array}{l}\text { RHODOMYRTOXIN } \\
\text { B }\end{array}$ & $\mathrm{C} 24 \mathrm{H} 28 \mathrm{O} 7$ & \multirow{5}{*}{$\begin{array}{l}\text { Anti } \\
\text { inflammatory } \\
\text { antiviral } \\
\text { (HIV) } \\
\text { Ecto } \\
\text { parasiticide }\end{array}$} & $\begin{array}{l}\text { Rhodomyrtus } \\
\text { macrocarpa }\end{array}$ & 234508 & 0.479 \\
\hline AUSTRICINE & $\mathrm{C} 15 \mathrm{H} 18 \mathrm{O} 4$ & & Compositae & 235476 & 0.505 \\
\hline BAICALEIN & $\mathrm{C} 15 \mathrm{H} 10 \mathrm{O} 5$ & & $\begin{array}{l}\text { Scutellaria } \\
\text { baicalensis }\end{array}$ & 236264 & 0.539 \\
\hline METHOPRENE (S) & $\mathrm{C} 19 \mathrm{H} 34 \mathrm{O} 3$ & & synthetic & 236431 & 0.486 \\
\hline $\begin{array}{l}\text { POSITIVE CONTROL } \\
\text { NEGATIVE CONTROL }\end{array}$ & & & & $\begin{array}{l}339962 \\
214974\end{array}$ & $\begin{array}{l}0.528 \\
0.064\end{array}$ \\
\hline Table 5.1: Spectrum & brare candt & non Alomen & donorintiono of & ounds & \\
\hline
\end{tabular}




\subsubsection{LOPAC Library against mtrA expression}

Using the established assay, we then screened the LOPAC library. Fortunately, this library contained an intrinsic positive control that we were not previously aware of. Oleic acid, which was briefly discussed as a TCS inhibitor in the introduction, was identified as a hit in the LOPAC screen, confirming our assay. Figure 5.2 displays the OD and $F$ readings for each well in this screen with oleic acid labelled in a different colour to show where hits of this screen should sit. As with the previous figure, the median values are shown as the axis lines to divide the graph into four quarters. The "hits" observed are displayed in table 5.2. Unfortunately in this assay, the variance in the negative controls was far more pronounced. Positive control wells displayed a huge range of variance in OD. However a trend can be seen from the top right quarter to the bottom left, in terms of the positive control readings, so the hits were selected according to their position away from this diagonal line.

The basis for choosing hits from this screen was their position in relation to the oleic acid, which is known to inhibit the phosphorylation step of HK dimerization. Ten compounds, named in table 5.2, were selected and re-tested in triplicate. 


\section{LOPAC screen with pSHigh_MtrA}

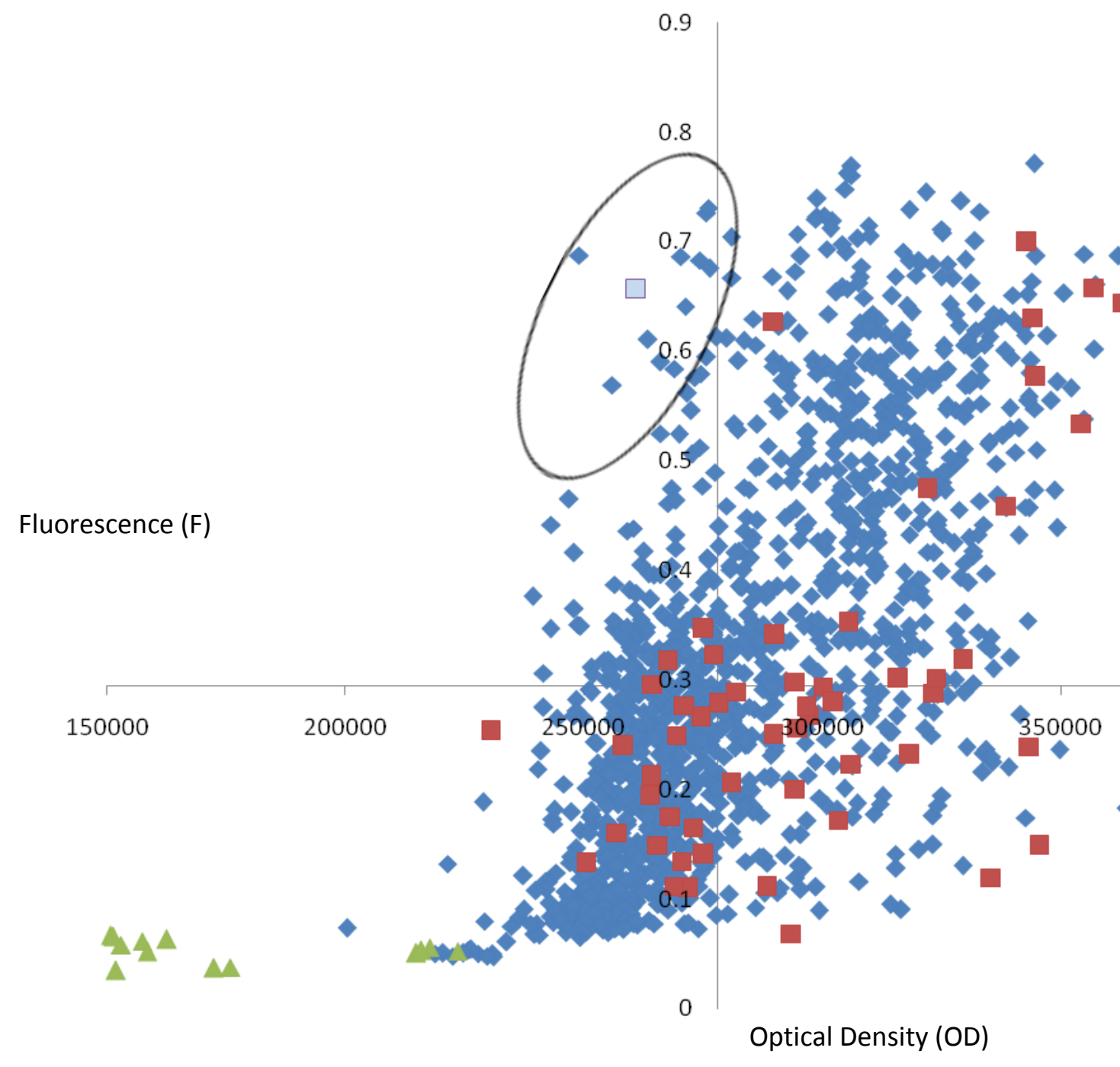

$\bullet$ LOPAC

a positive control $\triangle$ antibiotic control $\square$ Oleic acid
450000

Figure 5.2: LOPAC Screen against mtrA

expression. . Fluorescence and optical density are shown for each compound screened, using the median value as the axis. Oleic acid, a known TCS inhibitor is labelled in light blue. Ten other compounds, shown in a circle, were selected for re testing. Their names, a brief description as well as their values for $F$ and $O D$ are shown in table 5.2 


\begin{tabular}{|c|c|c|c|c|}
\hline NAME & Class & Description & $\mathrm{F}$ & OD \\
\hline $\begin{array}{l}\text { PROTRIPTYLINE } \\
\text { HYDROCHLORIDE }\end{array}$ & Adrenoceptor & $\begin{array}{l}\text { Norepinephrine reuptake } \\
\text { blocker }\end{array}$ & 243046 & 0.441 \\
\hline ODQ & Cyclic Nucleotides & $\begin{array}{l}\text { Potent and selective NO- } \\
\text { sensitive guanylyl cyclase } \\
\text { inhibitor }\end{array}$ & 246851 & 0.464 \\
\hline $\begin{array}{l}\mathrm{N} \text { - ACETYLPROCAINAMIDE } \\
\text { HYDROCHLORIDE }\end{array}$ & $\mathrm{Na}+$ Channel & $\begin{array}{l}\text { Blocks } \mathrm{Na}+\text { channels and } \\
\text { appears to have Class III anti- } \\
\text { arrhythmic activity; } \\
\text { metabolite of Procainamide }\end{array}$ & 247850 & 0.416 \\
\hline $\begin{array}{l}\text { PARGYLINE } \\
\text { HYDROCHLORIDE }\end{array}$ & Neurotransmission & $\begin{array}{l}\text { MAO inhibitor, relatively } \\
\text { selective for MAO-B }\end{array}$ & 249006 & 0.687 \\
\hline NILUtAMIDE & Hormone & Antiandrogen & 255868 & 0.569 \\
\hline OLEIC ACID & Phosphorylation & $\begin{array}{l}\text { Activates protein kinase } C \text { in } \\
\text { hepatocytes; uncouples } \\
\text { oxidative phosphorylation }\end{array}$ & 260912 & 0.656 \\
\hline $\begin{array}{l}\text { XANTHINE AMINE } \\
\text { CONGENER }\end{array}$ & Adenosine & $\begin{array}{l}\text { A1 Adenosine receptor } \\
\text { antagonist }\end{array}$ & 263300 & 0.610 \\
\hline L-ARGININE & Nitric Oxide & Nitric oxide precursor & 266069 & 0.590 \\
\hline $\begin{array}{l}\text { OXOTREMORINE } \\
\text { METHIODIDE }\end{array}$ & Cholinergic & $\begin{array}{l}\text { Nonselective muscarinic } \\
\text { acetylcholine receptor agonist }\end{array}$ & 266106 & 0.52 \\
\hline $\begin{array}{l}\text { S- (P-AZIDOPHENACYL) } \\
\text { GLUTATHIONE }\end{array}$ & $\begin{array}{l}\text { Multi-Drug } \\
\text { Resistance }\end{array}$ & $\begin{array}{l}\text { Glyoxalase and glutathione S- } \\
\text { transferase inhibitor }\end{array}$ & 269002 & 0.583 \\
\hline Positive Control & & & 296366 & 0.290 \\
\hline Negative Control & & & 171356 & 0.053 \\
\hline
\end{tabular}

Table 5.2 LOPAC Library Candidates. Names and descriptions of compounds considered for re-testing from $L O P A C$ collection with their $F$ and $O D$ values from the initial screen. This information was obtained from Sigma.

\subsubsection{NIH Library against $m t r A$ expression}

The NIH screen was the largest compound library tested in this study. This library has mostly been used to test potential anti-neoplastic agents and can be obtained from the NIH. It includes a large number of unknown compounds as well as a number of natural products. Figure 5.3 displays the $F$ and OD of each compound tested with median values used as the $\mathrm{X}$ and $\mathrm{Y}$ axis in each case. Positive control values are mostly isolated to the top right hand corner, while antibiotic control values are in the bottom left. Hits from the top left hand corner were selected for re-testing. 
Table 5.3 shows the names (if known), formula, molecular weight and $F$ and OD values of those compounds labelled "hits" for this screen. This information was obtained from the Developmental Therapeutics Programme (DTP) website which can be found at http://www.dtp.nci.nih.gov/docs/dtp search.html.

Most of the compounds labelled as hits for this screen have not been named, with the exception of Antineoplastic-92893 and Buclizine Hydrochloride, as shown in table 5.3. Consequently, there was little information available about these compounds other than their structure and formula. 


\section{NIH collection screen with pSHigh_MtrA}

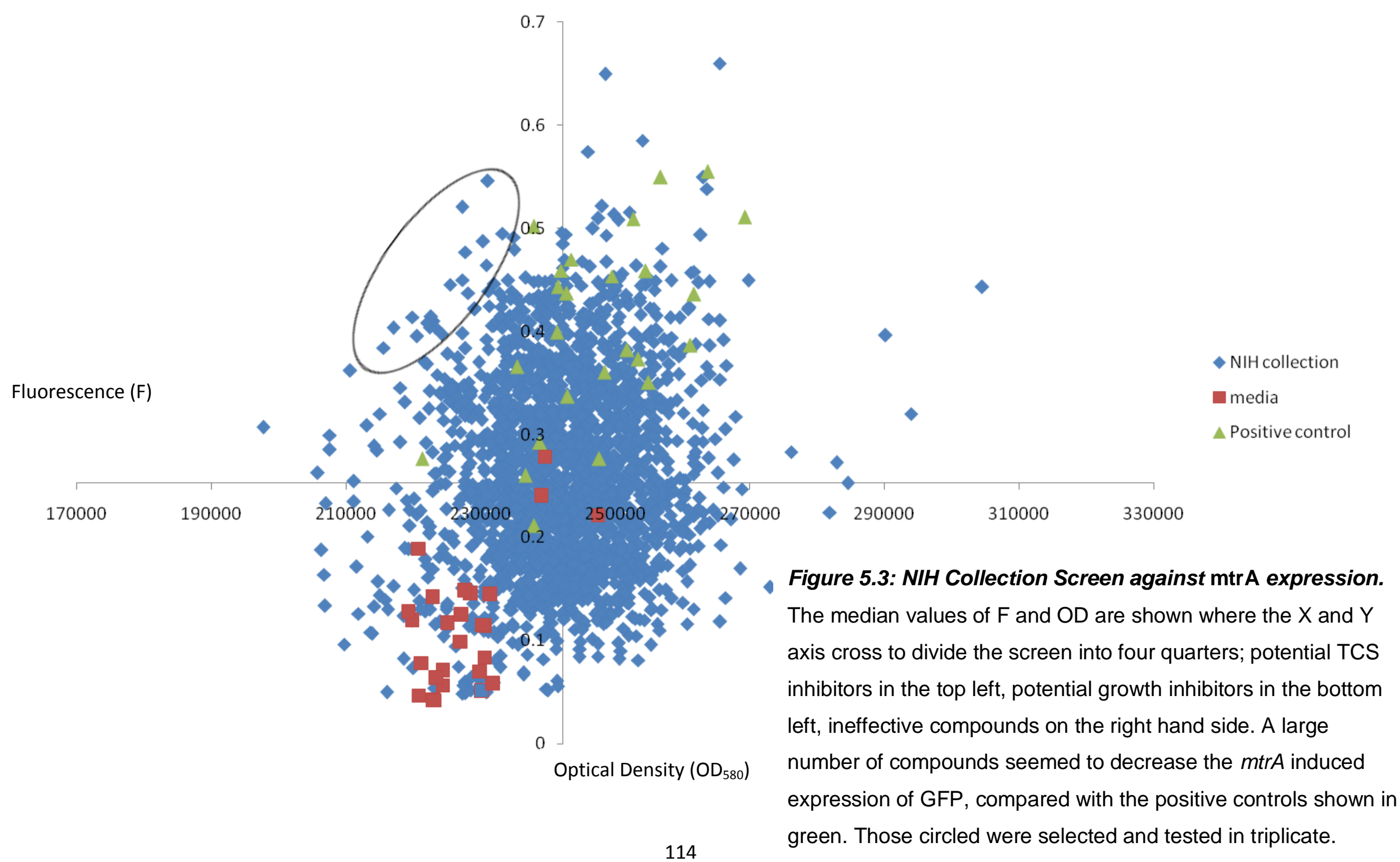




\begin{tabular}{|c|c|c|c|c|c|}
\hline Name & NIH code & Formula & $\begin{array}{l}\text { Molecular } \\
\text { Weight }\end{array}$ & $\mathbf{F}$ & OD \\
\hline & NSC 73646 & $\mathrm{C}_{6} \mathrm{H}_{16} \mathrm{~N}_{4} \cdot \mathrm{H}_{2} \mathrm{O}_{4} \mathrm{~S}$ & 242.0 & 210551 & 0.361 \\
\hline & NSC 96606 & $\mathrm{C}_{12} \mathrm{H}_{14} \mathrm{~N}_{2} \mathrm{O}_{2}$ & 218.0 & 234878 & 0.261 \\
\hline & NSC 63786 & $\mathrm{C}_{10} \mathrm{H}_{10} \mathrm{~N}_{4} \mathrm{~S}$ & 218.0 & 244330 & 0.216 \\
\hline & NSC 3535 & $\mathrm{C}_{13} \mathrm{H}_{19} \mathrm{NO}_{2} \mathrm{~S}$ & 253.0 & 215539 & 0.383 \\
\hline & $\begin{array}{l}\text { NSC } \\
133896\end{array}$ & $\mathrm{C}_{13} \mathrm{H}_{6} \mathrm{~N}_{2} \mathrm{O}_{5}$ & 270.0 & 227222 & 0.520 \\
\hline & $\begin{array}{l}\text { NSC } \\
130915\end{array}$ & $\mathrm{C}_{12} \mathrm{H}_{21} \mathrm{~N}_{3} \cdot 2 \mathrm{ClH}$ & 280.0 & 219812 & 0.413 \\
\hline \multirow{5}{*}{$\begin{array}{l}\text { Antineoplastic- } \\
92893\end{array}$} & NSC 92893 & $\mathrm{C}_{13} \mathrm{H}_{14} \mathrm{AsNO}_{3}$ & 307.0 & 217123 & 0.403 \\
\hline & $\begin{array}{l}\text { NSC } \\
166638\end{array}$ & $\mathrm{C}_{1}{ }_{0} \mathrm{H}_{7} \mathrm{C}_{12} \mathrm{~F}_{3} \mathrm{~N}_{4} \mathrm{O}_{2}$ & 343.0 & 228152 & 0.437 \\
\hline & NSC 97845 & $\mathrm{C}_{22} \mathrm{H}_{27} \mathrm{NO}_{3}$ & 353.0 & 231052 & 0.546 \\
\hline & $\begin{array}{l}\text { NSC } \\
357777\end{array}$ & $\mathrm{C}_{19} \mathrm{H}_{15} \mathrm{~N}_{5} \mathrm{O} . \mathrm{ClH}$ & 366.0 & 233207 & 0.495 \\
\hline & NSC 18877 & $\mathrm{C}_{20} \mathrm{H}_{10} \mathrm{~N}_{2} \mathrm{O}_{4} \mathrm{~S}_{2}$ & 406.0 & 227708 & 0.476 \\
\hline \multirow{3}{*}{$\begin{array}{l}\text { Buclizine } \\
\text { Hydrochloride }\end{array}$} & NSC 25141 & $\mathrm{C}_{28} \mathrm{H}_{33} \mathrm{ClN}_{2 .} 2 \mathrm{ClH}$ & 506.0 & 225434 & 0.445 \\
\hline & $\begin{array}{l}\text { NSC } \\
115448\end{array}$ & $\mathrm{C}_{44} \mathrm{H}_{20} \mathrm{~N}_{2} \mathrm{O}_{8}$ & 705.0 & 227322 & 0.449 \\
\hline & $\begin{array}{l}\text { NSC } \\
382796\end{array}$ & $\mathrm{C}_{31} \mathrm{H}_{25} \mathrm{~N}_{3} \mathrm{O}_{7}$ & 552.0 & 230822 & 0.464 \\
\hline $\begin{array}{l}\text { Positive control } \\
\text { (average) }\end{array}$ & & & & 247467 & 0.385 \\
\hline $\begin{array}{l}\text { Negative } \\
\text { control } \\
\text { (average) }\end{array}$ & & & & 217468 & 0.056 \\
\hline
\end{tabular}
re-testing with their $F$ and $O D$ values from the initial screen. 


\subsubsection{NIWA collection against $m$ trA expression}

A collection of marine extracts was also tested against the $m$ trA promoter. This collection contained a smaller number of samples than any other library and displayed no decrease in $\mathrm{F}$ while maintaining the OD of the culture compared with positive controls, as displayed in figure 5.4. The antibiotic controls indicate that a number of extracts had an effect on the growth of cells. All the other libraries used a constant concentration of $20 \mu \mathrm{M}$ for all compounds but the NIWA collection was composed of crude marine extracts, so the final concentration of active compounds is unknown. 


\section{NIWA collection screen with pSHigh_MtrA}

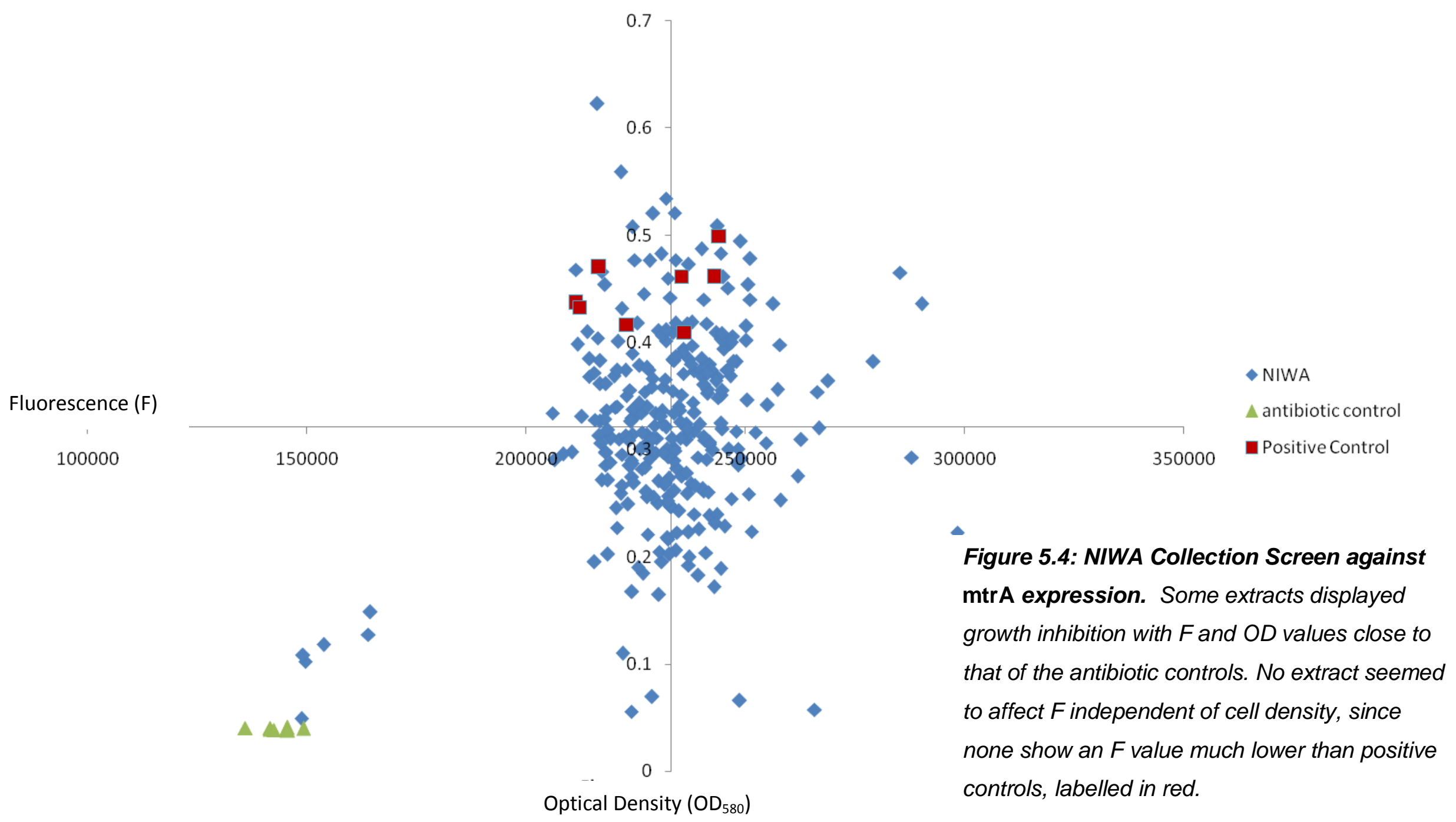




\subsubsection{Drug screens with pSHigh_phoA}

The phoA promoter-driven F of GFP is relatively lower than that of MtrA. This presented a problem, as the variance in $\mathrm{F}$ between wells was very high. For library screens with phoA, an additional negative control was used to indicate $\mathrm{F}$ of the promoterless pSHigh plasmid construct in the same EDTA supplemented media. An un-induced phoA control was not really practical in this screen, since these cells would grow at a different rate to the cells grown in EDTA supplemented media. There were no significant results seen in this screen, as changes in intensity between positive and negative controls could not be discerned.

\subsubsection{LOPAC library screen with phoA}

Figure 5.5 shows the phoA activation and OD against the LOPAC library. Although the position of the oleic acid control is in the top left quarter, as expected, a large number of compounds seem to cause a decrease in $\mathrm{F}$ to levels lower than the promoterless control. This could indicate that the use of EDTA imposes conditions which make the cells more susceptible to compounds that would not necessarily give the same result in rich media. This construct was also used to screen against the NIWA collection with a similar result (this data is not shown). 


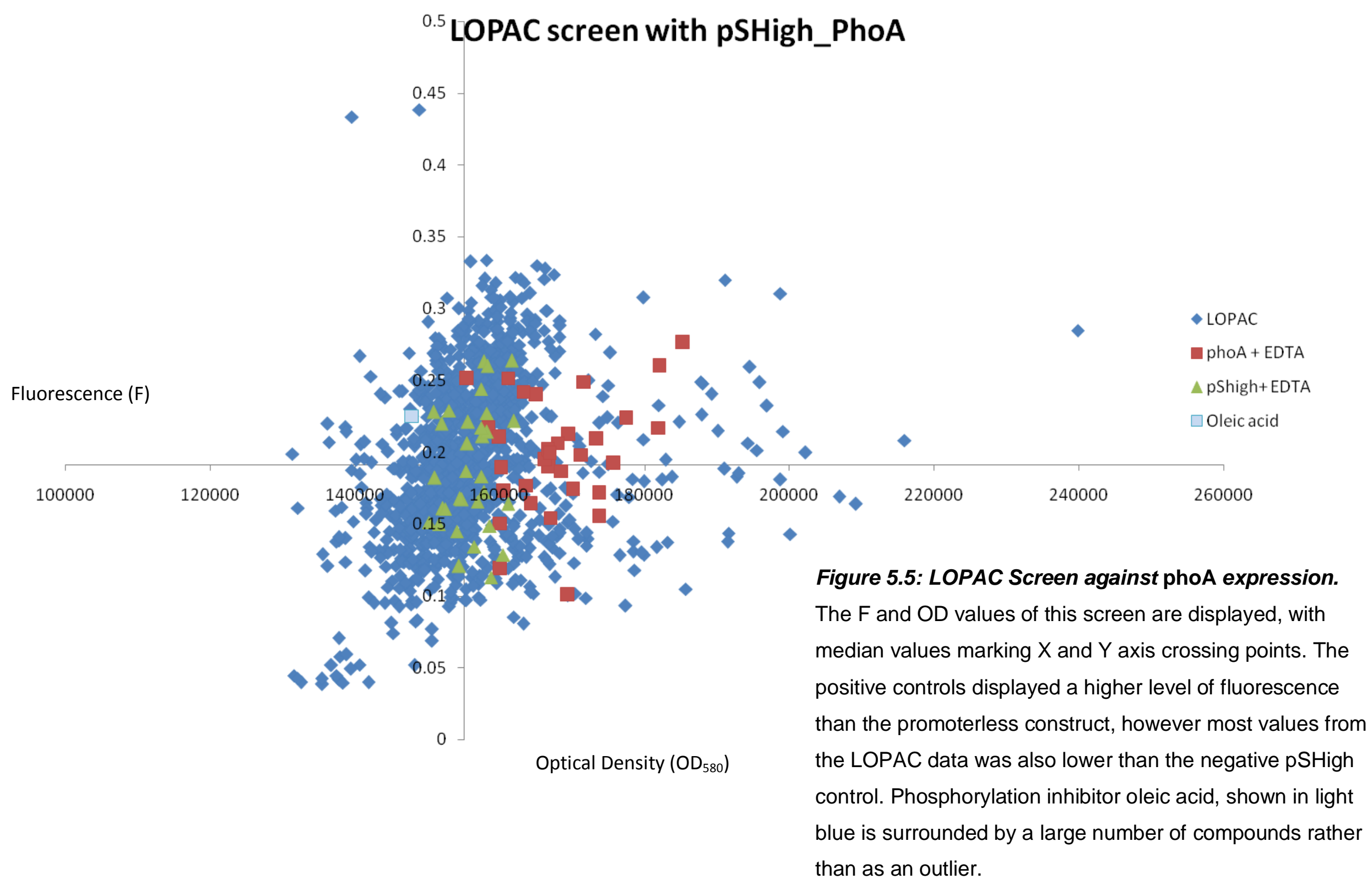




\subsubsection{Comparison of $m$ trA and phoA LOPAC screens:}

Even though the phoA screen was not successful in showing any hits, the oleic acid control was where we expected it to be; the top left hand quarter of the graph. In order to determine whether any of the proposed hits from the mtrA LOPAC screen fell in this quadrant during the phoA screen they have been represented on the same graph as the previous figure 5.5 in a different colour. If any of the compounds highlighted in the mtrA screen were not specific to the MtrB/ MtrA TCS and could inhibit different TCS as well, they might show up in the top left hand corner of the phoA screen as well. Figure 5.6 shows the same data as figure 5.5, with the hits from the mtrA screen highlighted in red and oleic acid in black and labelled.

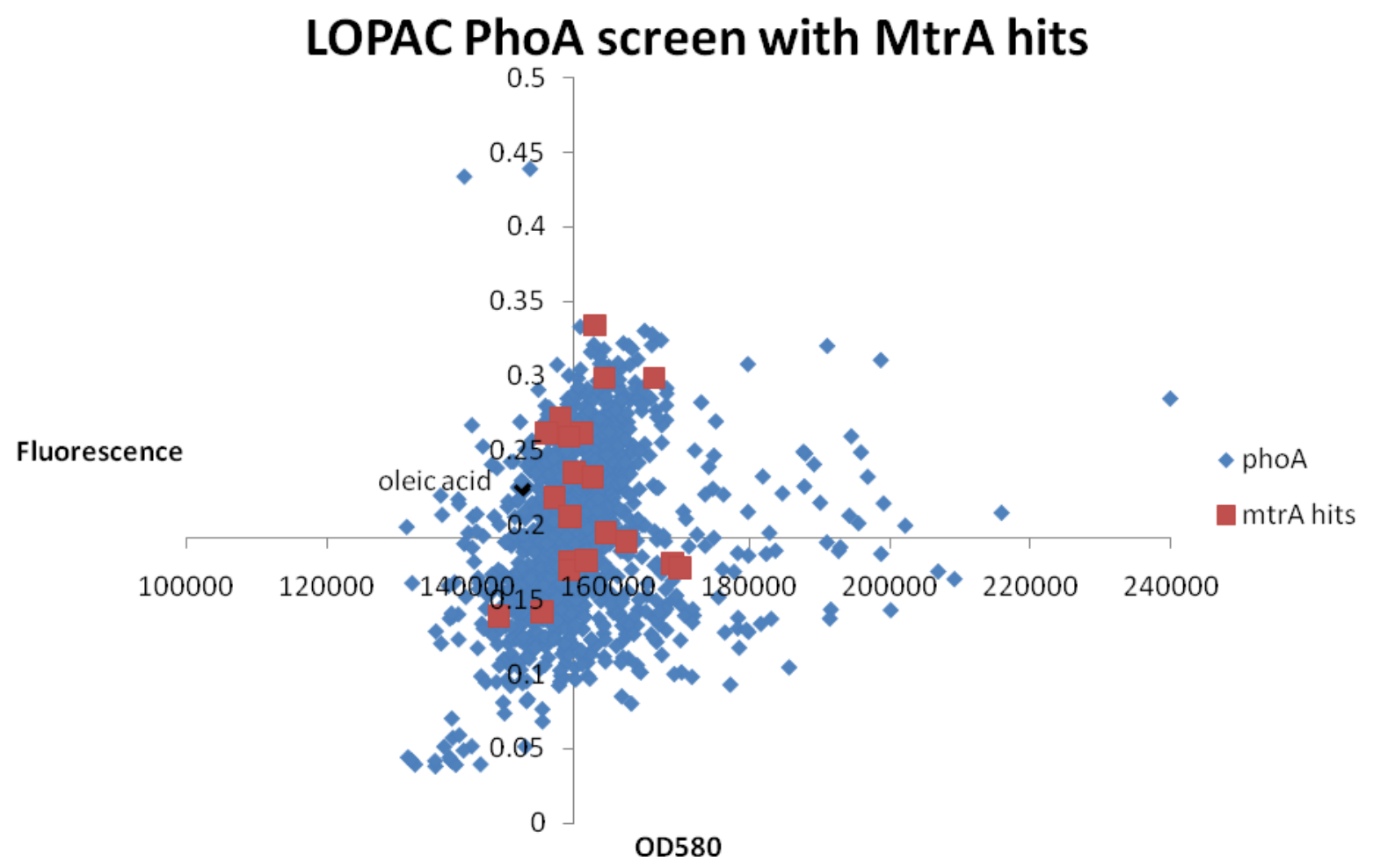

Figure 5.6: Identification of mtrA hits on LOPAC Screen against phoA. The $F$ and $O D$ values of this screen are displayed, with median values marking $X$ and $Y$ axis crossing points. Compounds which gave a lower $F$ reading in the mtrA screen are shown in red. A few values lie in the bottom left hand corner, indicating they may inhibit growth under the conditions of this screen, however none lie far to the left of the top corner which would signify that they are nonspecific TCS inhibitors. 
Only one of the compounds from the LOPAC screens showed inhibition of both mtrA and phoA driven promoter expression. This indicated that any of the other potential inhibitors identified during the mtrA screen were specific to this one out of the two signal relay system tested. It is also likely that this method of extrapolating hits may inadvertently overlook compounds with TCS inhibitory properties because only a small number of points were selected from each graph. Another factor is that compounds which conferred a low $\mathrm{F}$ reading, as well as a low $\mathrm{OD}_{580}$ reading are left out, as it was assumed these compounds are cytotoxic.

\subsubsection{Validation of hits from library screens in triplicate}

In order to verify that the compounds identified as hits from the library screens inhibited mtrA activation, selected compounds from each screen (named in tables $5.1-5.4)$ were re-tested in triplicate. For this validation assay the positive control promoter hsp60 and the promoterless pSHigh plasmids were included. The positive control would show whether the effect of the compound was a result of a global decrease of gene expression in the cell, or via an MtrA specific mechanism. Only one of the compounds tested in triplicate, oleic acid, displayed a reproducible effect on $m$ trA expression. They also displayed no change with respect to $h s p 60$ expression, indicating that hits selected from each screen had been false positive results and not inhibitors of gene expression. 


\subsubsection{Validation of whole cell based screen with oleic acid and palmitoleic acid dilution series}

None of the compounds picked up as hits in the library screens gave a significant decrease in $m t r A$ expression during a validation screen, where each compound was tested in triplicate. In order to show that this screen could potentially identify TCSi, had any been present in the libraries tested, a series of oleic acid and palmitoleic acid concentrations were tested for modulation of $m$ trA activation. Figures 5.7 and 5.8 show the $m t r A$ expression in the presence of palmitoleic and oleic respectively at three different concentrations of the fatty acids. These graphs show that the $m \operatorname{tr} A$ promoter driven GFP expression decreases with the concentration of both oleic and palmitoleic acid added, while the effect on the $h s p 60$ promoter expression is unchanged. 
Oleic Acid Concentration and mtrA Expression

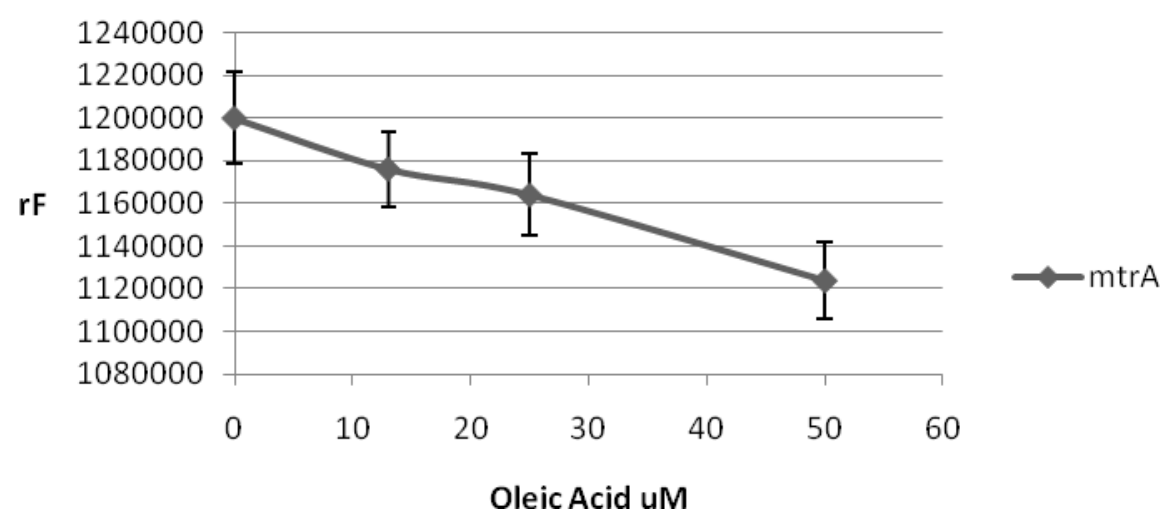

\section{Oleic Acid Concentration and hsp60} Expression

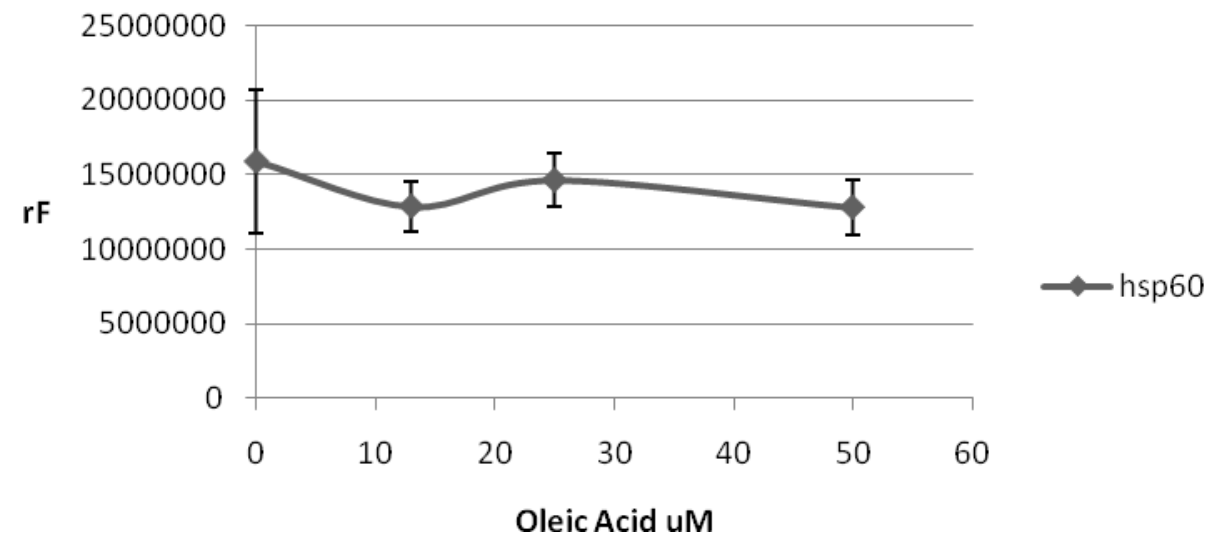

Figure 5.7: Oleic Acid dilution series against mtrA expression. A) MtrA and B) hsp60 expression under the influence of the oleic acid. The effect of final concentrations of 50, 25 and $12.5 \mu \mathrm{M}$ of oleic acid on the expression of mtrA is dose dependent as the $F$ of the reporter decreases with concentration, while hsp60 expression does not change with concentration. Error bars indicate the standard deviation between triplicate cultures. 


\section{Palmitoleic Acid Concentration and mtrA Expression}

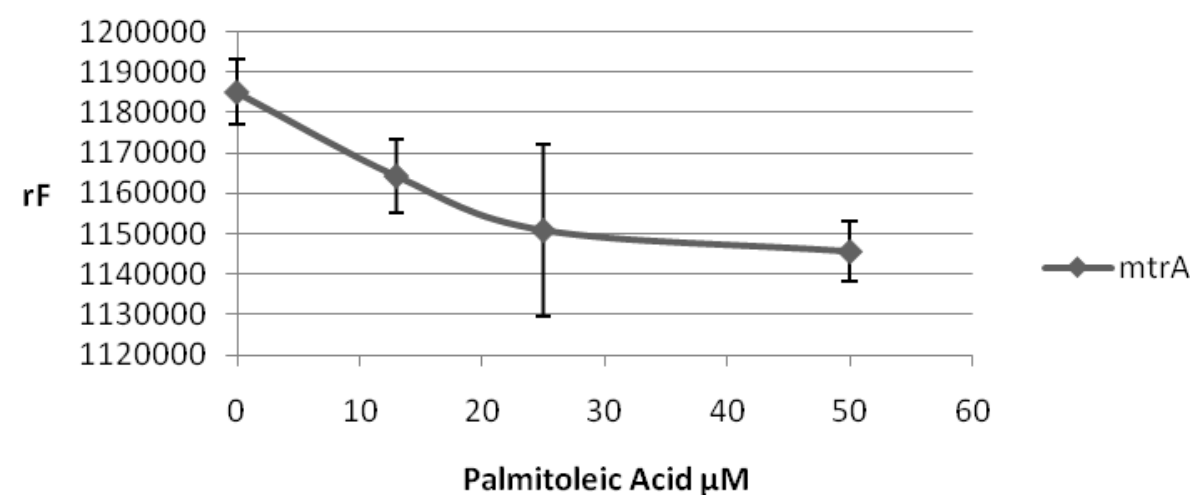

\section{Palmitoleic acid Concentration and hsp60 Expression}

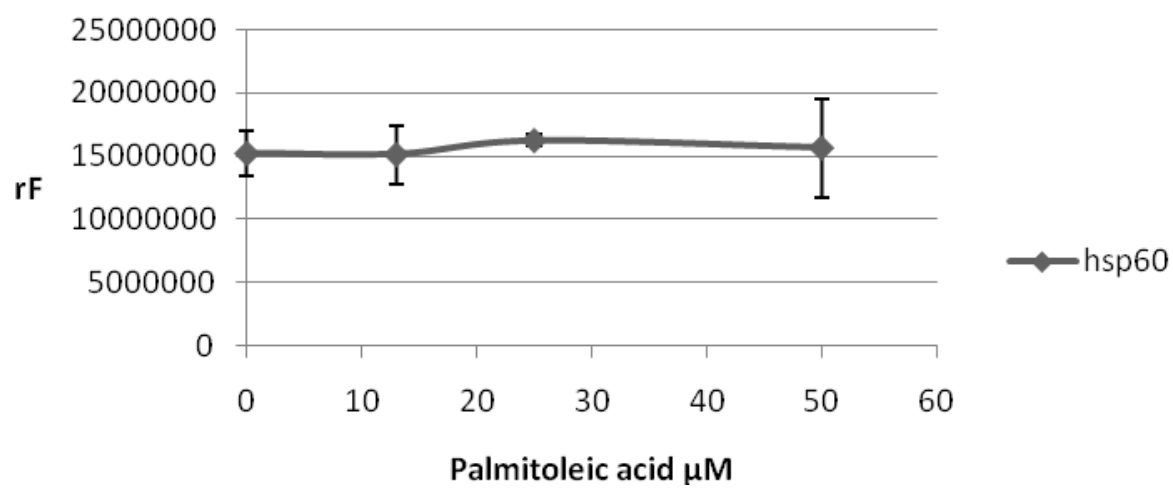

Figure 5.8: Palmitoleic Acid dilution series against mtrA expression. A) MtrA and B) hsp60 induction under the influence of the palmitoleic acid. The effect of final concentrations of 50, 25 and $12.5 \mu \mathrm{M}$ of palmitoleic acid on the expression of mtrA is dose dependent as the $F$ of the reporter decreases with concentration, while hsp60 expression does not change with concentration. A solvent control of ethanol is shown as $0 \mu \mathrm{M}$ palmitoleic acid. Error bars indicate the standard deviation between triplicate cultures. 


\subsection{Discussion}

\subsubsection{Compound Library screens and TCS controlled gene expression}

\subsubsection{Spectrum Collection}

A total of 2000 compounds from the Spectrum library were tested for activity against the expression of the mtrA promoter. Figure 5.1 shows that compounds had an array of effects on both $\mathrm{OD}$ and $\mathrm{F}$. The positive controls of M.smegmatis containing pSHigh_mtrA remain in the top right hand corner of the graph, indicating no reduction in $\mathrm{F}$ or $\mathrm{OD}$, while antibiotic controls shown in the bottom left corner indicate background $\mathrm{F}$ and turbidity produced by media without cells. The compounds have a range of effects with a main cluster halfway between these controls. The general trend shows that a number of compounds effect the growth of cells to some extent. The cluster of data points that is lower than the positive controls indicates that the presence of most compounds has some affect on cells either acting on the promoter activity or outright cell growth. Compounds that inhibit cell growth are present near the antibiotic control points. This graph depicts a number of hits which have a low level of $\mathrm{F}$ and no change in OD compared with positive controls.

\subsubsection{NIH Collection}

The NIH collection is a free library of compounds available from NCBI. 2240 compounds were tested from this library including a number from the natural diversity set. Unlike the Spectrum screen the positive controls are not all located in the top right hand quarter of the graph; however there is a clear trend between 
positive and negative controls. A total of 15 hits were selected for validation from this screen.

\subsubsection{NIWA samples}

A selection of marine samples donated by the National Institute of Water and Atmospheric Research (NIWA) in Wellington were also tested in this manner. This screen contained 290 samples, none of which gave an $\mathrm{F}$ reading lower than the positive controls. It did produce a number of data points with $\mathrm{F}$ and OD inhibition which will be used to verify future work with this library in a search for antimycobacterial drugs.

\subsubsection{LOPAC library}

The LOPAC collection, Library of Pharmacologically Active Compounds, available from Sigma Aldrich includes 1280 compounds. This screen was carried out for both $m$ trA and phoA driven GFP expression. Ten compounds were selected and retested with the mtrA construct. A major benefit of this library screen was the presence of a phosphorylation inhibitor oleic acid, displayed separately on figures 5.5 and 5.6. This compound was identified as a hit for the $m t r A$ screen, conveniently providing an intrinsic control to our assay. The phoA screen shows oleic acid in the top left quarter of the graph but not as a hit as in the $m t r A$ screen.

The phoA screen was less successful in that the difference between EDTA induced pSHigh_phoA and promoterless pSHigh was not well defined. A majority of the data points fell below this negative control, indicating there was some other interaction occurring between compounds and GFP expression. 
Interestingly none of the $m t r A$ hits identified were common to inhibitors on the phoA screen, indicating the library search had not successfully unearthed a non-specific inhibitor of HK to RR phosphotransfer. A recent study has highlighted the relationship between drug efficacy and the conditions in which cells are grown ${ }^{142}$. It has already been established that an anti-TB drug metronidazole is much more effective in hypoxic conditions ${ }^{159}$. These considerations add a level of complexity to drug screening as most in vitro drug screening is carried out in a specific media, and results can vary if these parameters are changed.

\subsubsection{Discussion on the Screen}

There a number of noteworthy limitations to this screen. Firstly the variance observed between samples when dealing with a multitude of compounds in this manner. The presence of outliers in both directions of the graph indicate that there is a significant chance of error that can come from a number of sources, from human handling and data processing error to technological errors such as pinning of compounds or reading of wells, the latter obviously far less likely. False positive "hits" may arise from a number of scenarios

- Contamination of a well in the assay which was not picked up upon inspection of plate, giving a high OD but no $\mathrm{F}$

- If the compound has an effect on the GFP protein processing at any point a misfolded protein may not give off the same $F$ as positive controls

- Coloured compounds can alter the OD reading of its well; a highly coloured compound may give a reading which indicates high cell viability when the chemical has in fact inhibited growth 
This data shows that the best results from the HTP screen did not translate to a decrease in $\mathrm{F}$ when repeated in triplicate. The high level of variance displayed in control and compound containing wells suggests that there is a loss of uniformity when working in a 96 wp set up in terms of OD and F. The validation results call into question the methods used during the HTP screen, and whether adequate parameters had been set. Given the high level of variance seen in a HTP screen with such a large number of compounds, the hits listed may either be false positives from the initial screens or too variable in the validation to be observed.

One concern with using the mtrA promoter in these screens stems from the fact that mtrA may be essential in M. tuberculosis. It has not been previously described as an essential gene in M. smegmatis; however it brings to light the concept that an inhibitor of this system may display an effect on cell growth if it is indeed essential. However, the data from both LOPAC screens and the oleic and palmitoleic acid experiments indicate that at a low concentration this inhibitor does not affect the cell density.

One way that this result may have been improved would have been to use another expression condition for mtrA; minimal media with EDTA. The decision to use rich media over minimal media stemmed from an observation that cells grew with much more uniformity in the former. As observed with the phoA promoter screens, minimal media gives a lower dynamic range making the difference between induced and uninduced GFP expression less pronounced. 


\subsubsection{Validation of the screen with cis fatty acids}

Figures 5.7 and 5.8 show a dose dependent decrease in $\mathrm{F}$ in the presence of oleic and palmitoleic acid. This trend is not seen in $\mathrm{F}$ which is restricted to the $m \operatorname{tr} A$ and not the $h s p 60$ promoter indicating that their mode of action is not on the GFP reporter itself or general global gene expression.

Oleic and palmitoleic acids are thought to inhibit phosphorylation of ATP to ADP in the histidine kinase activation ${ }^{160}$. They occur naturally in human, animal and plant fat and do not inhibit bacterial growth ${ }^{130}$. This activity was discovered in a kinase involved in sporulation named KinA of $B$. subtilis. The mode of action of cis unsaturated fatty acids is not known, except that they inhibit KinA -ATP binding in a non competitive fashion with an $\mathrm{IC}_{50}$ of around $20 \mu \mathrm{M}{ }^{160}$. Oleic acid is a component of mycobacterial media OADC, and must be broken down by the bacteria as an energy source, so its inhibitory activity may only act at a certain concentration, or in a particular arrangement of the fatty acid.

Oleic acid has also been described as an uncoupler of mitochondrial oxidative phosphorylation and an activator of protein kinase $C^{161,162}$. The full extent of its activity on phosphorylation reactions in mycobacteria is unknown.

This data shows in principal that using a serial dilution setup with three strains containing pSHigh_MtrA, pSHigh_hsp60 and pSHigh each in triplicate is a method which will illuminate TCSi from a library of compounds. The screen in this study aimed to test as many compounds as possible for activity; in other words, one compound per well at one concentration. This data clarifies the need for compounds to be tested in a dilution series and in triplicate. This method is more time consuming 
and probably reduces the number of compounds which can be tested per screen, but clearly yields a more reproducible result.

Previous work in HTS for active compounds has developed methods for statistical validation of primary screening ${ }^{142,163}$. This method determines the validity of hit selection in an assay based on a number of factors. In our assay only one hit was validated, indicating that this screen requires a significant amount of optimisation in order to obtain an acceptable hit rate or z score. 


\section{Chapter Six:}

\section{General Discussion and Future Directions}




\subsection{Summary of Findings}

The primary accomplishments of this thesis were the optimisation of a high copy number plasmid to report gene expression, the application of this methodology to TCS controlled genes and an analysis of the expression of these genes under stress. This led to a number of chemical library screens, in search of compounds which inhibit the TCS signal transduction. In theory, compounds could be potential TCS inhibitors if they exert a drop-off in TCS controlled gene expression compared with what is normally expected under particular conditions. Since they change the eventual outcome of the signalling mechanism i.e., the induction of a particular gene, these compounds could be affecting any part of the TCS signalling mechanism.

The insertion of the oriM high mycobacterial origin of a replication and the gfpmut2 gene resulted in the successful optimisation of a reporter plasmid. The results in Chapter Three demonstrate that the presence of the plasmid provides a high level of $F$ under the influence of the $h s p 60$ promoter without causing any obvious detrimental effects on $M$. smegmatis cells. This plasmid was further equipped with alternative promoters which were selected after a literature search of TCS controlled genes; namely $h s p X$, phoA and $m t r A$.

The activation of these genes was explored under a number of stress conditions including oxygen and nutrient starvation, antibiotic stress, heat shock, cold shock, SDS and ethanol. Out of all the stress conditions tested, the most noteworthy effect on the expression of $h s p X$ was seen during oxygen starvation. 
PhoA activation was observed under phosphate starvation and magnesium starvation, by chelation with EDTA. The mtrA promoter gave high levels of expression in rich media as well as under the influence of EDTA. The effect on $h s p X$ during oxygen starvation and phoA during phosphate starvation was expected given their proposed function. The induction of both $p h o A$ and $m t r A$ under the influence of magnesium starvation lead to a number of new questions about the influence of magnesium, or at least the chelator EDTA, in relation to the SenX3/ RegX3 and MtrB/ MtrA systems.

A number of assumptions have been made about the conditions experienced by AFB within the macrophage and granuloma which cannot be truly replicated in vitro. It is known that $m t r A$ is induced after macrophage infection in $M$. tuberculosis and this result brings to light the possibility that magnesium concentration or some other effect of the chelating agent on media may be involved in the MtrB sensor kinase activation.

Chemical library screens were carried out using the $m t r A$ reporter expression as an indicator of TCS inhibition, as this promoter produced high levels of F in rich media. A number of compounds decreased the expression of $m t r A$ after the incubation period of the library screens. Re-testing of these compounds in triplicate did not confirm a considerable difference in expression. We did, however, unearth a known TCSi in the LOPAC library which was identified as a hit in this screen. Further experiments on this compound and palmitoleic acid, another cis fatty acid, indicated that the $\mathrm{F}$ induced by $m t r A$ was reduced in a dose dependent manner in a serial dilution assay. 
Importantly, the screen identified an intrinsic TCSi control, oleic acid, out of thousands of compounds tested. This compound has not previously been shown to inhibit any TCS in mycobacteria.

\subsection{Critique of the screen}

There are a number of benefits to this screen compared with isolated protein methods. In theory any inhibition of the signal transduction mechanism will be highlighted by this screen. The method can be carried out in a high through put manner to test thousands of compounds at a time in one assay.

This method could be adapted to other targets. As well as finding inhibitors of transcriptional control, the use of GFP translational fusions can be applied to discover inhibitors of proteins which are controlled by other means.

A significant point here is the ability to test for inhibitors of genes that may be essential. Few methods can directly target a gene which the organism cannot live without, but the reduction in mtrA promoter activation observed as a result of oleic acid showed that changes in gene expression can be observed in this way without completely inhibiting cell growth. One the other hand, this will depend on the MIC of the inhibitor in question and the concentration of compounds used in a screen. In all likelihood a successful candidate drug which inhibits TCS would also decrease the cell density of a population and thus be missed out in our screen.

However, more potent inhibitors may not have been picked up by our methods. This situation could be remedied by a more relative measure of $F$, by recording information on a single cell basis with FACs. It comes down to a compromise between high throughput and relativity of gene expression. By using a whole cell 
assay thousands more compounds can be screened than in a low throughput single cell counting method such as FACS.

The use of a reporter of gene expression, however accurate, is essentially an indirect method of testing two component signal transduction. We do not know how many other regulators are acting on the genes in this study. Previous work has shown that all of the selected genes contained RR binding sites within their promoters, but this does not exclude the possibility of other factors such as universal sigma factors ${ }^{100,108,150}$. The mechanisms controlling gene expression are, in all likelihood, much more complicated than simply one response regulator acting as a transcription factor on a gene. There are probably multiple levels of regulation for every gene in an organism's genome. The constitutively expressed control promoter is one way of showing if a condition or inhibitor is affecting the expression of a specific gene; however in practice the control of these important housekeeping genes could be controlled another way.

Although the TCSi oleic acid was picked up this screen a number of other false positive results were also picked up using this method. Clearly the level of variance in terms of both F and OD observed from screens of this size can lead to false positive results and perhaps screens of this nature should be repeated before selecting hits to re-test. In order to test as many compounds as possible each library was only screened once. This meant that there was no way to test the statistical significance of "hits". In hindsight, this meant that the screen was not an efficient way of finding TCS inhibitors. If each library had been tested in triplicate it is likely that the rate of false positives would have been reduced. Hit selection is an area which requires significant development in order to obtain an acceptable z score. Our 
method for manually selecting compounds based on their position on the scatter plot did not render an acceptable hit rate as described in other work on HTS analysis ${ }^{163}$. Inevitably, the number of compounds being screened will affect the degree of accuracy each screen will present, since larger screens give more variable results. Perhaps the parameters of "hit" selection could be optimised.

One aspect of the screen, which was briefly mentioned earlier, is the relativity of results in terms of the number of plasmids per cell. As with all plasmid reporter methods, including $x y l E$ expression, one must assume that this remains relatively constant between strains and conditions. It is generally accepted that using appropriate controls will balance out any changes in copy number from cell to cell. It is worth noting here that oriM high increased copy number from 5 to anything between 32 and 64 copies per cell raises questions about this assumption of consistency. Large differences in copy number of the plasmid from cell to cell may be the cause of the variance seen in these screens. The benefits of this new origin of replication are convincing (Chapter One), but the resulting variance is something that needs to be considered.

In this study, over 4500 compounds from a number of sources were screened, a fair few less than the 25,000 compounds tested to discover the first TCS inhibitors ${ }^{127}$. A possible future direction in this area could be the study of fewer compounds after a more rational method of compound selection. For example, selecting compounds or classes of compounds identified using an in silico screening approach based on the protein structure of the target. The current work highlighted the potential of cis fatty acids, so perhaps this is a class of compounds worth developing. 


\subsection{Future Direction}

The identification of oleic acid in the LOPAC assay and the dose dependent inhibition displayed by oleic and palmitoleic acids indicate that this methodology could be applied to study more compound libraries against the reporter system. Before further libraries are tested, there is a substantial amount of optimisation required in terms of HTS. A number of statistical tests, for example, would help to indicate an improved hit rate as a result of a more refined selection criteria. As well as increasing the number of chemicals tested, there is also the possibility that new TCS controlled genes could be screened in this manner. Other $M$. smegmatis, $M$. bovis BCG or avirulent $M$. tuberculosis TCS controlled genes could be investigated using this method of screening.

The observation that cis fatty acids may inhibit the expression of both $m t r A$ and $p h o A$ is an important finding, as this inhibition has so far only been observed in the Bacillus subtilis KinA protein. The method of action has not yet been elucidated, except that fatty acids act in a non competitive manner with respect to ATP during phosphorylation. It would be interesting to solve the crystal structure of MtrB and compare it with that of KinA for homologous sites.

The large number of compounds screened was achieved in this study using a fast growing species of mycobacteria. Slow growers would require lengthy assays, but could also potentially be screened either with entire libraries or a selection of hits discovered by assays with fast growers. 
Another application of these methods is the study of genes induced within the macrophage. In particular, $m$ tr $A$ expression, which has been previously shown during macrophage expression, could induce GFP at a much higher level during intra-macrophage growth. If the difference between induced and un-induced GFP is more apparent, the level of variance seen in these screens could be reduced.

As well as TCS, other signalling such as Serine Threonine Protein Kinases and onecomponent regulators could be selected and screened for in this manner.

DevS/DevR and other dormancy related targets would be highly appropriate targets to develop in this sort of screen.

PhoP/ PhoR could also be targeted in this manner by analysing the expression of the gene under its control. The benefits of inhibiting this system would be plentiful, as it is so important to the survival and virulence of $M$. tuberculosis. Its significant role in cell wall maintenance would make any inhibitor capable of knocking out this signalling mechanism an excellent drug candidate. Even if it did not inhibit growth outright, its use in combination with other drugs such as cell wall targets could significantly reduce the treatment time required for TB.

Other parts of this thesis underline the need for further studies into the conditions of expression of TCS controlled genes. In particular $m$ trA; how and why this gene is expressed and its protein product's function within the cell are all important questions, due to its significance as an $M$. tuberculosis essential gene. This work has revealed a possible condition of its expression to be magnesium starvation. Further work to validate this may help to understand its function and potential as a drug target. 
There are generally two ways to go about discovering drug targets for a particular pathogen. We argue that a targeted approach to significant genes involved in latency, dormancy and survival within a macrophage cell may be a better way to prevent TB than exploring any and all compounds which affect the growth rate of the species. For a number of reasons, M. tuberculosis requires a different approach to drug discovery, compared with rapidly growing pathogens. The high incidence of drug resistance, its extended incubation and latency period and its apparent switch to a reductive metabolism make $M$. tuberculosis a difficult pathogen to eradicate. Screening for compounds, and selecting "essential" targets by measuring simply how they affect the level of growth of the organism in rich media is not the best representation of what the pathogens really cannot survive without in vivo. The $m t r A$ gene has been labelled essential, however, its role in macrophage infection has also been established, making it the sort of target which should be explored further.

Similarly, expression of the $h s p X$ reporter plasmid represents the switch to a reductive metabolism, something which makes the pathogen extremely successful. As a drug target, or an indicator of the cell dormancy program, this gene is highly useful in the study of latency. Compounds which affect this and other dormancy related targets could be worthy candidates in the effort to stop TB. 


\section{REFERENCES}

1. Myers, J.A., Bearman, J.E. \& Botkins, A.C. Natural History of Tuberculosis in the Human Body. Diseases of the Chest 50, 120-132 (1966).

2. Hershkovitz, I. et al. Detection and Molecular Characterization of 9000-Year-Old Mycobacterium tuberculosis from a Neolithic Settlement in the Eastern Mediterranean. PLoS ONE 3, e3426 (2008).

3. Chalke, H.D. The impact of Tuberculosis on History, Literature and Art. Med Hist 6, $301-318$ (1962).

4. Charles Greenblatt, M.S. Emerging Pathogens:Archaeology, Ecology and Evolution of Infectious Disease. Oxford University Press (2003).

5. Bruford, M.W., Bradley, D.G. \& Luikart, G. DNA markers reveal the complexity of livestock domestication. Nat Rev Genet 4, 900-910 (2003).

6. Perkins, D., Jr. Fauna of Catal Huyuk: Evidence for Early Cattle Domestication in Anatolia. Science 164, 177-179 (1969).

7. Diamond, J. Guns, Germs and Steel. Vintage, Random House (1997).

8. Russell, D.G. Who puts the tubercle in tuberculosis? Nat Rev Micro 5, 39-47 (2007).

9. Rothschild, B.A M. et al. Mycobacterium tuberculosis Complex DNA from an Extinct Bison Dated 17,000 Years before the Present. Clinical Infectious Diseases 33, 305-311 (2001).

10. Gutierrez, M.C. et al. Ancient Origin and Gene Mosaicism of the Progenitor of Mycobacterium tuberculosis. PLoS Pathog 1, e5 (2005).

11. Myers, J. Eighty Years After the First Glimpse of the Tubercle Bacillus. Diseases of the Chest 51, 500-519 (1967).

12. Tortoli, E. Impact of Genotypic Studies on Mycobacterial Taxonomy: the New Mycobacteria of the 1990s. Clin. Microbiol. Rev. 16, 319-354 (2003).

13. Basu, S. \& Galvani, A. The Evolution of Tuberculosis Virulence. Bulletin of Mathematical Biology.

14. Reyrat, J.-M. \& Kahn, D. Mycobacterium smegmatis: an absurd model for tuberculosis? Trends in Microbiology 9, 472-473 (2001).

15. Garnier, T. et al. The complete genome sequence of Mycobacterium bovis. Proc Natl Acad Sci USA 100, 7877 - 7882 (2003).

16. Thompson, D. Mass Immunization in the Control of Infectious Disease. British med J August 427 - 433 (1966).

17. Ducati, R.G., Ruffino-Netto, A., Basso, L.A. \& Santos, D.S. The resumption of consumption : a review on tuberculosis. Memórias do Instituto Oswaldo Cruz 101, 697-714 (2006).

18. Barr RG, M.R. The effect of war on tuberculosis. Results of a tuberculin survey among displaced persons in El Salvador and a review of the literature. Tuber Lung Dis 75, 251 - 259 (1994).

19. Kent, N. Tuberculosis. British med J September 673 -674 (1979).

20. Malley, G. Evolution of chemotherapy in Tuberculosis. AMA Arch Intern Med 93, 967-976 (1954).

21. Gomez, J.E. \& McKinney, J.D. M. tuberculosis persistence, latency, and drug tolerance. Tuberculosis 84, 29-44 (2004). 
22. Gillespie, S.H. Evolution of Drug Resistance in Mycobacterium tuberculosis: Clinical and Molecular Perspective. Antimicrob. Agents Chemother. 46, 267-274 (2002).

23. WHO Global Tuberculosis Control: Surveillance, Finance and Planing. WHO report World Health Organization Document (2009).

24. Vilcheze, C. \& Jacobs, J., William R. The Mechanism of Isoniazid Killing: Clarity Through the Scope of Genetics. Annual Review of Microbiology 61, 35-50 (2007).

25. Waksman, S.A. Streptomycin: Background, isolation, properties, and utilization. Nobel Lecture (1952).

26. Honore, N. \& Cole, S.T. Streptomycin resistance in mycobacteria. Antimicrob. Agents Chemother. 38, 238-242 (1994).

27. Abbadi, S.H., Sameaa, G.A., Morlock, G. \& Cooksey, R.C. Molecular identification of mutations associated with anti-tuberculosis drug resistance among strains of Mycobacterium tuberculosis. International Journal of Infectious Diseases In Press, Corrected Proof.

28. Guy, E.S. \& Mallampalli, A. Review: Managing TB in the 21st century: existing and novel drug therapies. Therapeutic Advances in Respiratory Disease 2, 401-408 (2008).

29. Wade, M.M. \& Zhang, Y. Anaerobic incubation conditions enhance pyrazinamide activity against Mycobacterium tuberculosis. J Med Microbiol 53, 769-773 (2004).

30. ESR New Zealand Public Health Surveillance Report December 2008: Covering July September 2008 December 2008: Covering July - September 2008. New Zealand Public Health Surveillance 6 (2008).

31. Dye, C., Watt, C., Bleed, D., Hosseini, S. \& Raviglione, M. Evolution of tuberculosis control and prospects for reducing tuberculosis incidence, prevalence, and deaths globally. JAMA 293, 2767 - 2775 (2005).

32. Dye, C. Doomsday postponed? Preventing and reversing epidemics of drug-resistant tuberculosis. Nat Rev Micro 7, 81-87 (2009).

33. Gilles Gonzalez, M.A., Gonzalez, G., Sousa, E.H.S., Tuckerman, J. \& Robert, K.P. in Methods in Enzymology, Vol. Volume 437 173-189 (Academic Press, 2008).

34. Connolly, L.E., Edelstein, P.H. \& Ramakrishnan, L. Why Is Long-Term Therapy Required to Cure Tuberculosis? PLoS Medicine 4, e120 (2007).

35. O'Sullivan, D.M., McHugh, T.D. \& Gillespie, S.H. The effect of oxidative stress on the mutation rate of Mycobacterium tuberculosis with impaired catalase/peroxidase function. $J$. Antimicrob. Chemother. 62, 709-712 (2008).

36. Balaban, N.Q., Merrin, J., Chait, R., Kowalik, L. \& Leibler, S. Bacterial Persistence as a Phenotypic Switch. Science 305, 1622-1625 (2004).

37. Howie, S. et al. Tuberculosis in New Zealand, 1992-2001: a resurgence. Arch Dis Child 90, 1157-1161 (2005).

38. Cardona, P. \& Ruiz-Manzano, J. On the nature of Mycobacterium tuberculosis-latent bacilli. Eur Respir J 24, 1044-1051 (2004).

39. Bishai, W. The Mycobacterium tuberculosis genomic sequence: anatomy of a master adaptor. Trends in Microbiology 6, 464-465 (1998).

40. Bhatt, K. \& Salgame, P. Host Innate Immune Response to Mycobacterium tuberculosis. Journal of Clinical Immunology 27, 347-362 (2007).

41. Manabe, Y.C. \& Bishai, W.R. Latent Mycobacterium tuberculosis-persistence, patience, and winning by waiting. Nat Med 6, 1327-1329 (2000).

42. Timo Ulrichs, S.H.K. New insights into the function of granulomas in human tuberculosis. The Journal of Pathology 208, 261-269 (2006).

43. Timo Ulrichs, S.K. Mycobacterial persistence and Immunity. Frontiers in Bioscience 7, 458469 (2002).

44. Cosma, C.L., Sherman, D.R. \& Ramakrishnan, L. The Secret Lives of the Pathogenic mycobacteria. Annual Review of Microbiology 57, 641-676 (2003). 
45. Davis, J.M. \& Ramakrishnan, L. The Role of the Granuloma in Expansion and Dissemination of Early Tuberculous Infection. Cell 136, 37-49 (2009).

46. Hotter, G. et al. Transposon mutagenesis of Mb0100 at the ppe1-nrp locus in Mycobacterium bovis disrupts phthiocerol dimycocerosate (PDIM) and glycosylphenol-PDIM biosynthesis, producing an avirulent strain with vaccine properties at least equal to those of M. bovis BCG. J Bacteriol 187, 2267 - 2277 (2005).

47. Wayne, L.G. Dormancy of Mycobacterium tuberculosis and latency of disease. European Journal of Clinical Microbiology \& Infectious Diseases 13, 908-914 (1994).

48. Boon, C. \& Dick, T. Mycobacterium bovis BCG Response Regulator Essential for Hypoxic Dormancy. J. Bacteriol. 184, 6760-6767 (2002).

49. Hernández-Pando, R. et al. Persistence of DNA from Mycobacterium tuberculosis in superficially normal lung tissue during latent infection. The Lancet 356, 2133-2138 (2000).

50. Lillebaek, T. et al. Stability of DNA patterns and evidence of Mycobacterium tuberculosis reactivation occurring decades after the initial infection. J Infect Dis 188, 1032 - 1039 (2003).

51. Keane, J. et al. Tuberculosis Associated with Infliximab, a Tumor Necrosis Factor alpha Neutralizing Agent. N Engl J Med 345, 1098-1104 (2001).

52. Stein, C.M. et al. Genome Scan of M. tuberculosis Infection and Disease in Ugandans. PLoS ONE 3, e4094 (2008).

53. Smeulders, M.J., Keer, J., Speight, R.A. \& Williams, H.D. Adaptation of Mycobacterium smegmatis to Stationary Phase. J. Bacteriol. 181, 270-283 (1999).

54. Woolhiser, L. et al. In Vivo Adaptation of the Wayne Model of Latent Tuberculosis. Infect. Immun. 75, 2621-2625 (2007).

55. Wayne, L.G. Synchronized replication of Mycobacterium tuberculosis. Infect. Immun. 17, 528-530 (1977).

56. Wayne, L.G. \& Lin, K.Y. Glyoxylate metabolism and adaptation of Mycobacterium tuberculosis to survival under anaerobic conditions. Infect. Immun. 37, 1042-1049 (1982).

57. Honer Zu Bentrup, K., Miczak, A., Swenson, D.L. \& Russell, D.G. Characterization of Activity and Expression of Isocitrate Lyase in Mycobacterium avium and Mycobacterium tuberculosis. J. Bacteriol. 181, 7161-7167 (1999).

58. Demangel, C., Garnier, T., Rosenkrands, I. \& Cole, S. Differential effects of prior exposure to environmental mycobacteria on vaccination with Mycobacterium bovis BCG or a recombinant BCG strain expressing RD1 antigens. Infect Immun 73, 2190 - 2196 (2005).

59. Ming C. Tsai, S.C., Guofeng Zhu, Jiayong Xu, Kathryn Tanaka, Cameron Koch, JoAnn Tufariello, JoAnne Flynn, John Chan, Characterization of the tuberculous granuloma in murine and human lungs: cellular composition and relative tissue oxygen tension. Cellular Microbiology 8, 218-232 (2006).

60. Sardiwal, S. et al. A GAF Domain in the Hypoxia/NO-inducible Mycobacterium tuberculosis DosS Protein Binds Haem. Journal of Molecular Biology 353, 929-936 (2005).

61. Saini, D. et al. DevR-DevS is a bona fide two-component system of Mycobacterium tuberculosis that is hypoxia-responsive in the absence of the DNA-binding domain of DevR. Microbiology 150, 865 - 875 (2004).

62. O'Toole, R. et al. A Two-Component Regulator of Universal Stress Protein Expression and Adaptation to Oxygen Starvation in Mycobacterium smegmatis. J. Bacteriol. 185, 1543-1554 (2003).

63. Mayuri, Bagchi, G., Das, T.K. \& Tyagi, J.S. Molecular analysis of the dormancy response in Mycobacterium smegmatis: expression analysis of genes encoding the DevR-DevS twocomponent system, Rv3134c and chaperone [alpha]-crystallin homologues. FEMS Microbiology Letters 211, 231-237 (2002).

64. Starck, J., Kallenius, G., Marklund, B.-I., Andersson, D.I. \& Akerlund, T. Comparative proteome analysis of Mycobacterium tuberculosis grown under aerobic and anaerobic conditions. Microbiology 150, 3821-3829 (2004). 
65. Boshoff, H.I. \& Barry lii, C.E. Is the mycobacterial cell wall a hopeless drug target for latent tuberculosis? Drug Discovery Today: Disease Mechanisms 3, 237-245 (2006).

66. Wang, J.Y. \& Koshland, D.E., Jr Evidence for protein kinase activities in the prokaryote Salmonella typhimurium. J. Biol. Chem. 253, 7605-7608 (1978).

67. Cashin, P., Goldsack, L., Hall, D. \& O'Toole, R. Contrasting signal transduction mechanisms in bacterial and eukaryotic gene transcription. FEMS Microbiology Letters 261, 155-164 (2006).

68. Pohl, E., Holmes, R.K. \& Hol, W.G.J. Crystal Structure of the Iron-dependent Regulator (IdeR) from Mycobacterium tuberculosis Shows Both Metal Binding Sites Fully Occupied. Journal of Molecular Biology 285, 1145-1156 (1999).

69. Dussurget, $O$. et al. Transcriptional Control of the Iron-Responsive fxbA Gene by the Mycobacterial Regulator IdeR. J. Bacteriol. 181, 3402-3408 (1999).

70. Gold, B., Rodriguez, G.M., Marras, S.A.E., Pentecost, M. \& Smith, I. The Mycobacterium tuberculosis IdeR is a dual functional regulator that controls transcription of genes involved in iron acquisition, iron storage and survival in macrophages. Molecular Microbiology 42, 851 (2001).

71. Rodriguez, G.M., Voskuil, M.I., Gold, B., Schoolnik, G.K. \& Smith, I. ideR, an Essential Gene in Mycobacterium tuberculosis: Role of IdeR in Iron-Dependent Gene Expression, Iron Metabolism, and Oxidative Stress Response. Infect. Immun. 70, 3371-3381 (2002).

72. Ranjan, S. \& Ranjan, A. Bioinformatics Study of Iron Dependent Transcriptional Regulator, IdeR, from Mycobacteria. International Journal of Infectious Diseases 12, e479-e479 (2008).

73. Priska Peirs, L.W., Martine Braibant, Kris Huygen, Jean Content, A Serine/Threonine Protein Kinase from Mycobacterium tuberculosis. European Journal of Biochemistry 244, 604-612 (1997).

74. Koul, A. et al. Serine/threonine protein kinases PknF and PknG of Mycobacterium tuberculosis: characterization and localization. Microbiology 147, 2307-2314 (2001).

75. Av-Gay, Y. \& Everett, M. The eukaryotic-like Ser/Thr protein kinases of Mycobacterium tuberculosis. Trends in Microbiology 8, 238-244 (2000).

76. Kallipolitis, B. \& Ingmer, H. Listeria monocytogenes response regulators important for stress tolerance and pathogenesis. FEMS Microbiol Lett 204, 111 - 115 (2001).

77. Wehenkel, A. et al. Mycobacterial Ser/Thr protein kinases and phosphatases: Physiological roles and therapeutic potential. Biochimica et Biophysica Acta (BBA) - Proteins \& Proteomics 1784, 193-202 (2008).

78. Guangchun Bai, D.D.S., Kathleen A. McDonough, cAMP levels within Mycobacterium tuberculosis and Mycobacterium bovis BCG increase upon infection of macrophages. FEMS Immunology \& Medical Microbiology 55, 68-73 (2009).

79. Michaela A. Gazdik, G.B., Yan Wu, Kathleen A. McDonough, Rv1675c (cmr) regulates intramacrophage and cyclic AMP-induced gene expression in <i>Mycobacterium tuberculosis</i>-complex mycobacteria. Molecular Microbiology 71, 434-448 (2009).

80. Shpakov, A. \& Pertseva, M. Signal transduction systems in prokaryotes. Journal of Evolutionary Biochemistry and Physiology 44, 129-150 (2008).

81. Kundig, W. \& Roseman, S. Sugar Transport. I. Isolation of a phosphotransferase system from Escherichia coli. J. Biol. Chem. 246, 1393-1406 (1971).

82. AM, B., LA, A., BR, C. \& MI, S. Structure of CheA, a signal-transducing histidine kinase. Cell 96, 131 (1999).

83. Mizuno, T. Compilation of All Genes Encoding Two-component Phosphotransfer Signal Transducers in the Genome of Escherichia coli. DNA Res 4, 161-168 (1997).

84. Mizuno, T. His-Asp Phosphotransfer Signal Transduction. J Biochem 123, 555-563 (1998).

85. Wolanin, P. \& Stock, J. Transmembrane signaling and the regulation of histidine kinase activity In Histidine Kinases in Signal Transduction: abstract. (forthcoming review).

86. J. Tyagi, D.S. Signal transduction systems of mycobacteria with special reference to $M$. tuberculosis. Current Science 86, 93-102 (2004). 
87. Esther Pérez, S.S., Yann Bordas, Christophe Guilhot, Brigitte Gicquel, Carlos Martín, An essential role for phoP in Mycobacterium tuberculosis virulence. Molecular Microbiology 41, 179-187 (2001).

88. Friedland, N. et al. Domain Orientation in the Inactive Response Regulator Mycobacterium tuberculosis MtrA Provides a Barrier to Activation. Biochemistry 46, 6733-6743 (2007).

89. Patricia A. Fontan, S.W.a.I.S. Cellular signaling pathways and transcriptional regulation in Mycobacterium tuberculosis: Stress control and virulence. Current Science 88, 122-134 (2004).

90. Rong Gao, Y.T., Ann M. Stock, System-level mapping of Escherichia coli response regulator dimerization with FRET hybrids. Molecular Microbiology 69, 1358-1372 (2008).

91. Laub, M.T. \& Goulian, M. Specificity in Two-Component Signal Transduction Pathways. Annual Review of Genetics 41, 121-145 (2007).

92. Dortay, H. et al. Toward an Interaction Map of the Two-Component Signaling Pathway of Arabidopsis thaliana. Journal of Proteome Research 7, 3649-3660 (2008).

93. Rong Gao, Y.T., Ann M. Stock, System-level mapping of Escherichia coli response regulator dimerization with FRET hybrids. Molecular Microbiology 69, 1358-1372 (2008).

94. Amemura, M., Makino, K., Shinagawa, H. \& Nakata, A. Cross talk to the phosphate regulon of Escherichia coli by PhoM protein: PhoM is a histidine protein kinase and catalyzes phosphorylation of PhoB and PhoM-open reading frame 2. J. Bacteriol. 172, 6300 (1990).

95. Matsushita, M. \& Janda, K.D. Histidine kinases as targets for new antimicrobial agents. Bioorganic \& Medicinal Chemistry 10, 855-867 (2002).

96. Stephenson, K. \& Hoch, J.A. Virulence- and antibiotic resistance-associated two-component signal transduction systems of Gram-positive pathogenic bacteria as targets for antimicrobial therapy. Pharmacology \& Therapeutics 93, 293-305 (2002).

97. Stephenson, K. \& Hoch, J. Virulence- and antibiotic resistance-associated two-component signal transduction systems of Gram-positive pathogenic bacteria as targets for antimicrobial therapy. Pharmacol Ther 93, 293 - 305 (2002).

98. Watanabe, T., Okada, A., Gotoh, Y. \& Utsumi, R. in Bacterial Signal Transduction: Networks and Drug Targets 229-2362008).

99. R. Curcic, S.D., V. Oeretic, Gene expression in mycobacteria: transcriptional fusions based on $x y I E$ and analysis of the promoter region of the response regulator $m$ trA from Mycobacterium tuberculosis. Molecular Microbiology 13, 1057-1064 (1994).

100. Via, L. et al. Elements of signal transduction in Mycobacterium tuberculosis: in vitro phosphorylation and in vivo expression of the response regulator MtrA. J. Bacteriol. 178, 3314-3321 (1996).

101. Zahrt, T.C. \& Deretic, V. An Essential Two-Component Signal Transduction System in Mycobacterium tuberculosis. J. Bacteriol. 182, 3832-3838 (2000).

102. Marek Fol, A.C., Naveen K. Nair, Erin Maloney, Meredith Moomey, Chinnaswamy Jagannath, Murty V. V. S. Madiraju, Malini Rajagopalan, Modulation of <i>Mycobacterium tuberculosis</i> proliferation by MtrA, an essential two-component response regulator. Molecular Microbiology 60, 643-657 (2006).

103. Friedland, N. et al. Domain Orientation in the Inactive Response Regulator Mycobacterium tuberculosis MtrA Provides a Barrier to Activation\&\#x2020;,\&\#x2021. Biochemistry 46, 67336743 (2007).

104. M. Marques, M.A., Chitale, S., Brennan, P.J. \& Pessolani, M.C.V. Mapping and Identification of the Major Cell Wall-Associated Components of Mycobacterium leprae. Infect. Immun. 66, 2625-2631 (1998).

105. Cangelosi, G.A. et al. The Two-Component Regulatory System mtrAB Is Required for Morphotypic Multidrug Resistance in Mycobacterium avium. Antimicrob. Agents Chemother. 50, 461-468 (2006). 
106. Nina Möker, M.B., Steffen Schaffer, Reinhard Krämer, Susanne Morbach, Michael Bott, Deletion of the genes encoding the MtrA/MtrB two-component system of Corynebacterium glutamicum has a strong influence on cell morphology, antibiotics susceptibility and expression of genes involved in osmoprotection. Molecular Microbiology 54, 420-438 (2004).

107. Wang, W. et al. Molecular and biochemical characterization of a novel two-component signal transduction system, amrA-amkA, involved in rifamycin SV production in Amycolatopsis mediterranei U32. Archives of Microbiology 178, 376-386 (2002).

108. Glover, R.T., Kriakov, J., Garforth, S.J., Baughn, A.D. \& Jacobs, W.R., Jr. The Two-Component Regulatory System senX3-regX3 Regulates Phosphate-Dependent Gene Expression in Mycobacterium smegmatis. J. Bacteriol. 189, 5495-5503 (2007).

109. Parish, T. et al. Deletion of two-component regulatory systems increases the virulence of Mycobacterium tuberculosis. Infect Immun 71, 1134 - 1140 (2003).

110. Himpens, S., Locht, C. \& Supply, P. Molecular characterization of the mycobacterial SenX3RegX3 two-component system: evidence for autoregulation. Microbiology 146, 3091-3098 (2000).

111. Kriakov, J., Lee, S.h. \& Jacobs, W.R., Jr. Identification of a Regulated Alkaline Phosphatase, a Cell Surface-Associated Lipoprotein, in Mycobacterium smegmatis. J. Bacteriol. 185, 49834991 (2003).

112. Haydel, S.E. \& Clark-Curtiss, J.E. Global expression analysis of two-component system regulator genes during Mycobacterium tuberculosis growth in human macrophages. FEMS Microbiology Letters 236, 341-347 (2004).

113. Magdalena, J., Supply, P. \& Locht, C. Specific Differentiation between Mycobacterium bovis BCG and Virulent Strains of the Mycobacterium tuberculosis Complex. J. Clin. Microbiol. 36, 2471-2476 (1998).

114. Hasan, S., Daugelat, S., Rao, P.S.S. \& Schreiber, M. Prioritizing Genomic Drug Targets in Pathogens: Application to Mycobacterium tuberculosis. PLoS Computational Biology 2, e61 (2006).

115. Yukl, E.T., Ioanoviciu, A., Ortiz de Montellano, P.R. \& Moenne-Loccoz, P. Interdomain Interactions within the Two-Component Heme-Based Sensor DevS from Mycobacterium tuberculosis. Biochemistry 46, 9728-9736 (2007).

116. Via, L.E. et al. Tuberculous Granulomas Are Hypoxic in Guinea Pigs, Rabbits, and Nonhuman Primates. Infect. Immun. 76, 2333-2340 (2008).

117. Converse, P.J. et al. The role of the dosR/dosS two-component regulatory system in Mycobacterium tuberculosis virulence in three animal models. Infect. Immun., 1117-1108 (2008).

118. O'Toole, R. \& Williams, H. Universal stress proteins and Mycobacterium tuberculosis. Res Microbiol 154, 387 - 392 (2003).

119. Lyon, G.J., Mayville, P., Muir, T.W. \& Novick, R.P. Rational design of a global inhibitor of the virulence response in Staphylococcus aureus, based in part on localization of the site of inhibition to the receptor-histidine kinase, Agrc. Proceedings of the National Academy of Sciences of the United States of America 97, 13330-13335 (2000).

120. Wang, L., Grau, R., Perego, M. \& Hoch, J.A. A novel histidine kinase inhibitor regulating development in Bacillus subtilis. Genes \& Development 11, 2569-2579 (1997).

121. Bick, M.J. et al. How to Switch Off a Histidine Kinase: Crystal Structure of Geobacillus stearothermophilus KinB with the inhibitor Sda. Journal of Molecular Biology 386, 163-177 (2009).

122. Stephenson, K., Yamaguchi, Y. \& Hoch, J.A. The Mechanism of Action of Inhibitors of Bacterial Two-component Signal Transduction Systems. J. Biol. Chem. 275, 38900-38904 (2000). 
123. Foster, J.E. et al. Kinetic and mechanistic analyses of new classes of inhibitors of twocomponent signal transduction systems using a coupled assay containing HpkA-DrrA from Thermotoga maritima. Microbiology 150, 885-896 (2004).

124. Hilliard, J.J., Goldschmidt, R.M., Licata, L., Baum, E.Z. \& Bush, K. Multiple Mechanisms of Action for Inhibitors of Histidine Protein Kinases from Bacterial Two-Component Systems. Antimicrob. Agents Chemother. 43, 1693-1699 (1999).

125. Hlasta, D.J. et al. Novel inhibitors of bacterial two-component systems with gram positive antibacterial activity: Pharmacophore identification based on the screening hit closantel. Bioorganic \& Medicinal Chemistry Letters 8, 1923-1928 (1998).

126. Qin, Z. et al. Structure-based discovery of inhibitors of the YycG histidine kinase: New chemical leads to combat Staphylococcus epidermidis infections. BMC Microbiology 6, 96 (2006).

127. Roychoudhury, S. et al. Inhibitors of two-component signal transduction systems: inhibition of alginate gene activation in Pseudomonas aeruginosa. Proceedings of the National Academy of Sciences of the United States of America 90, 965-969 (1993).

128. Saini, D.K. \& Tyagi, J.S. High-Throughput Microplate Phosphorylation Assays Based on DevRDevS/Rv2027c 2-Component Signal Transduction Pathway to Screen for Novel Antitubercular Compounds. J Biomol Screen 10, 215-224 (2005).

129. Macielag, M. \& Goldschmidt, R. Inhibitors of bacterial two-component signalling systems. Expert Opin Investig Drugs 9, 2351 - 2369 (2000).

130. Barrett, J.F. et al. Antibacterial agents that inhibit two-component signal transduction systems. Proceedings of the National Academy of Sciences of the United States of America 95, 5317-5322 (1998).

131. Watanabe, T. et al. Isolation and characterization of inhibitors of the essential histidine kinase, YycG in Bacillus subtilis and Staphylococcus aureus. J Antibiot (Tokyo) 56, 1045 - 1052 (2003).

132. J. Dezz Ropp, C.J.D., David Wolfgang-Kimball, Jeffrey J. Hooley, James Y. W. Chin, Robert A. Hoffman, R. Andrew Cuthbertson, Kenneth D. Bauer, Aequorea green fluorescent protein analysis by flow cytometry. Cytometry 21, 309-317 (1995).

133. Chalfie M, T.Y., Euskirchen G, Ward WW, Prasher DC Green fluorescent protein as a marker for gene expression. Science 11, 802-805 (1994).

134. Blokpoel, M.C.J., O'Toole, R., Smeulders, M.J. \& Williams, H.D. Development and application of unstable GFP variants to kinetic studies of mycobacterial gene expression. Journal of Microbiological Methods 54, 203-211 (2003).

135. Laurent Kremer, A.B., Jerome Estaquier, Odile Poulain-Godefroy, Camille Locht. Green fluorescent protein as a new expression marker in mycobacteria. Molecular Microbiology 17, 913-922 (1995).

136. Cormack, B.P., Valdivia, R.H. \& Falkow, S. FACS-optimized mutants of the green fluorescent protein (GFP). Gene 173, 33-38 (1996).

137. Kenney, T. \& Churchward, G. Genetic analysis of the Mycobacterium smegmatis rpsL promoter. J. Bacteriol. 178, 3564-3571 (1996).

138. Bourn, W.R. et al. Creation and characterisation of a high-copy-number version of the pAL5000 mycobacterial replicon. Tuberculosis 87, 481-488 (2007).

139. O'Toole, R., Von Hofsten, J., Rosqvist, R., Olsson, P.E. \& Wolf-Watz, H. Visualisation of zebrafish infection by GFP-labelled Vibrio anguillarum. Microb Pathog 37, 41-46 (2004).

140. Sambrook, R. Molecular Cloning: A Laboratory Manual Cold Spring Harbor Laboratory Press, third edition. (2001).

141. Tanya Parish, N.S. Mycobacteria Protocols, Vol. 101. (Humana Press, 2008).

142. Miller, C.H., Nisa, S., Dempsey, S., Jack, C. \& O'Toole, R. Modifying Culture Conditions in Chemical Library Screening Identifies Alternative Inhibitors of Mycobacteria. Antimicrob. Agents Chemother. 53, 5279-5283 (2009). 
143. Kendall, S.L. et al. The Mycobacterium tuberculosis dosRS two-component system is induced by multiple stresses. Tuberculosis 84, 247-255 (2004).

144. Kamakshi Sureka, S.D., Pratik Datta, Anil Kumar Singh, Arunava Dasgupta, Sébastien Rodrigue, Joyoti Basu, Manikuntala Kundu, Polyphosphate kinase is involved in stressinduced mprAB-sigE-rel signalling in mycobacteria. Molecular Microbiology 65, 261-276 (2007).

145. Zahrt, T.C. \& Deretic, V. Mycobacterium tuberculosis signal transduction system required for persistent infections. Proceedings of the National Academy of Sciences of the United States of America 98, 12706-12711 (2001).

146. Walters, S. et al. The Mycobacterium tuberculosis PhoPR two-component system regulates genes essential for virulence and complex lipid biosynthesis. Mol Microbiol 60, 312 - 330 (2006).

147. Ewann, F., Locht, C. \& Supply, P. Intracellular autoregulation of the Mycobacterium tuberculosis PrrA response regulator. Microbiology 150, 241-246 (2004).

148. Marek Fol, A.C., Naveen K. Nair, Erin Maloney, Meredith Moomey, Chinnaswamy Jagannath, Murty V. V. S. Madiraju, Malini Rajagopalan, Modulation of Mycobacterium tuberculosis proliferation by MtrA, an essential two-component response regulator. Molecular Microbiology 60, 643-657 (2006).

149. Arun, K.H.S., Kaul, C.L. \& Ramarao, P. Green fluorescent proteins in receptor research: An emerging tool for drug discovery. Journal of Pharmacological and Toxicological Methods 51, $1-23$ (2005).

150. Chauhan, S. \& Tyagi, J.S. Cooperative Binding of Phosphorylated DevR to Upstream Sites Is Necessary and Sufficient for Activation of the Rv3134c-devRS Operon in Mycobacterium tuberculosis: Implication in the Induction of DevR Target Genes. J. Bacteriol. 190, 4301-4312 (2008).

151. Vasudeva-Rao, H.M. \& McDonough, K.A. Expression of the Mycobacterium tuberculosis acrCoregulated Genes from the DevR (DosR) Regulon Is Controlled by Multiple Levels of Regulation. Infect. Immun. 76, 2478-2489 (2008).

152. Kendall, S. et al. The Mycobacterium tuberculosis dosRS two-component system is induced by multiple stresses. Tuberculosis (Edinb ) 84, 247 - 255 (2004).

153. Betts, J., Lukey, P., Robb, L., McAdam, R. \& Duncan, K. Evaluation of a nutrient starvation model of Mycobacterium tuberculosis persistence by gene and protein expression profiling. Mol Microbiol 43, 717 - 731 (2002).

154. Kumar, A. et al. Heme Oxygenase-1-derived Carbon Monoxide Induces the Mycobacterium tuberculosis Dormancy Regulon. J. Biol. Chem. 283, 18032-18039 (2008).

155. Kumar, A., Toledo, J.C., Patel, R.P., Lancaster, J.R. \& Steyn, A.J.C. Mycobacterium tuberculosis DosS is a redox sensor and DosT is a hypoxia sensor. Proceedings of the National Academy of Sciences 104, 11568-11573 (2007).

156. Voskuil, M.I. et al. Inhibition of Respiration by Nitric Oxide Induces a Mycobacterium tuberculosis Dormancy Program. J. Exp. Med. 198, 705-713 (2003).

157. Ryndak, M., Wang, S. \& Smith, I. PhoP, a key player in Mycobacterium tuberculosis virulence. Trends in Microbiology 16, 528-534 (2008).

158. Cappelli, G. et al. Profiling of Mycobacterium tuberculosis gene expression during human macrophage infection: Upregulation of the alternative sigma factor $G$, a group of transcriptional regulators, and proteins with unknown function. Res Microbiol (2006).

159. Klinkenberg, S., Bishai, Karakousis Metronidazole Lacks Activity against Mycobacterium tuberculosis in an In Vivo Hypoxic Granuloma Model of Latency. The Journal of Infectious Diseases 198, 275-283 (2008).

160. Mark A. Strauch, D.M., James A. Hoch, cis-Unsaturated fatty acids specifically inhibit a signaltransducing protein kinase required for initiation of sporulation in Bacillus subtilis. Molecular Microbiology 6, 2909-2917 (1992). 
161. Murakami, K., Chan, S. \& Routtenberg, A. Protein kinase $C$ activation by cis-fatty acid in the absence of Ca2+ and phospholipids. J. Biol. Chem. 261, 15424-15429 (1986).

162. Buelt, M., Shekels, L., Jarvis, B. \& Bernlohr, D. In vitro phosphorylation of the adipocyte lipidbinding protein (p15) by the insulin receptor. Effects of fatty acid on receptor kinase and substrate phosphorylation. J. Biol. Chem. 266, 12266-12271 (1991).

163. Zhang, J.-H., Chung, T.D.Y. \& Oldenburg, K.R. Confirmation of Primary Active Substances from High Throughput Screening of Chemical and Biological Populations: A Statistical Approach and Practical Considerations. Journal of Combinatorial Chemistry 2, 258-265 (2000). 


\section{APPENDICES}

\section{Appendix 1: PCR reactions}

The PCR reactions carried out in this study have been displayed below in A.1.

Generally PCRs were carried in out using one of the three protocols depending on the desired fragment length and fidelity of PCR required. High Fidelity polymerase was used to clone promoters, gfpmut2 and oriM high. In the case of screening for fragment insertion into a plasmid, a regular polymerase was used such as Mastermix (Bioline). Modifications to annealing temperatures were made depending on particular primer melting temperatures in some cases.

\section{A.1 PCR reactions in this study}

a) High Fidelity PCR: Standard protocol for amplifying promoters and gfpmut2

\begin{tabular}{|c|c|c|}
\hline Temperature & Time & Cycles \\
\hline $94^{\circ} \mathrm{C}$ & 10 minutes & \\
\hline $94^{\circ} \mathrm{C}$ & 1 minute & \\
\hline $56^{\circ} \mathrm{C}$ & 1 minute & 30 \\
\hline $68^{\circ} \mathrm{C}$ & 1 minute & \\
\hline $12^{\circ} \mathrm{C}$ & hold & \\
\hline
\end{tabular}


b) High Fidelity PCR: Gradient protocol for amplifying long fragment oriM high

\begin{tabular}{|c|c|c|}
\hline Temperature & Time & Cycles \\
\hline $94^{\circ} \mathrm{C}$ & 10 minutes & \\
\hline $94^{\circ} \mathrm{C}$ & 1 minute & \\
\hline $55-65^{\circ} \mathrm{C}$ & 1 minute & 30 \\
\hline $68^{\circ} \mathrm{C}$ & 1 minute & \\
\hline $68^{\circ} \mathrm{C}$ & 10 seconds & \\
\hline $12^{\circ} \mathrm{C}$ & hold & \\
\hline
\end{tabular}

c) Standard PCR: Screening fragment insertion using pTKmx_F1 primer

\begin{tabular}{|c|c|c|}
\hline Temperature & Time & Cycles \\
\hline $94^{\circ} \mathrm{C}$ & 10 minutes & \\
\hline $94^{\circ} \mathrm{C}$ & 1 minute & \\
\hline $44^{\circ} \mathrm{C}$ & 1 minute & 30 \\
\hline $72^{\circ} \mathrm{C}$ & 1 minute & \\
\hline $72^{\circ} \mathrm{C}$ & 10 seconds & \\
\hline $12^{\circ} \mathrm{C}$ & hold & \\
\hline
\end{tabular}




\section{Appendix 2: Ligation Reactions}

A number of ligation reactions were carried out in this screen. The method used was altered depending on whether the reaction involved one or two restriction enzyme sites. Since the pSHigh plasmid had only one cloning site infront of gfpmut2 the plasmid was first incubated with CIP to reduce the amount of self ligation occurring in the reaction. Consequently a larger volume of plasmid was added to these reactions as the concentration was reduced by the DNA clean up step after CIP incubation. The volume of fragment added was also increase to give a more efficient reaction by increase the fragment to plasmid ratio. In addition the efficiency off each reaction was observed by carrying out a negative control with distilled water, as the number of colonies on the negative control plate were indicative of self ligation of the plasmid. The following tables (A.2 a, b and c) describe the stock concentrations and volumes of reagents used in this study for cloning into a) cloning vectors, b) pOT71 and c) pSHigh. 
A.2 Ligation Reactions used in this study

\begin{tabular}{lll}
\hline & Stock Concentration & In a $10 \mu \mathrm{L}$ reaction \\
\hline Promoter fragment & variable & $7 \mu \mathrm{L}$ \\
TA cloning vector & $1 \mathrm{ng}$ & $1 \mu \mathrm{L}$ \\
T4 ligase & $0.4 \mathrm{U} / \mu \mathrm{L}$ & $1 \mu \mathrm{L}$ \\
T4 ligase buffer & $10 \mathrm{x}$ & $1 \mu \mathrm{L}$ \\
\hline
\end{tabular}

a) Ligation reaction for cloning vectors

\begin{tabular}{lll}
\hline & $\begin{array}{l}\text { Stock } \\
\text { Concentration }\end{array}$ & $\begin{array}{l}\text { In a } 10 \mu \mathrm{L} \\
\text { reaction }\end{array}$ \\
\hline $\begin{array}{l}\text { Promoter fragment or SDW } \\
\text { Reporter plasmid pOT71 (after digestion } \\
\text { with EcoRI and BamHI) }\end{array}$ & variable & $7 \mu \mathrm{L}$ \\
T4 ligase & $0.4 \mathrm{U} / \mu \mathrm{L}$ & $1 \mu \mathrm{L}$ \\
T4 ligase buffer & $10 \mathrm{x}$ & $1 \mu \mathrm{L}$ \\
Kpnl & $1 \mathrm{U} / \mu \mathrm{L}$ & $1 \mu \mathrm{L}$ \\
\hline
\end{tabular}

b) Ligation reaction for pOT71

\begin{tabular}{lll}
\hline & $\begin{array}{l}\text { Stock } \\
\text { Concentration }\end{array}$ & $\begin{array}{l}\text { In a } 10 \mu \mathrm{L} \\
\text { reaction }\end{array}$ \\
\hline $\begin{array}{l}\text { Promoter fragment or SDW } \\
\text { with Kpnl and incubation with CIP) }\end{array}$ & variable & $20-30 \mu \mathrm{L}$ \\
T4 ligase & variable & $2 \mu \mathrm{L}$ \\
T4 ligase buffer & $0.4 \mathrm{U} / \mu \mathrm{L}$ & $2 \mu \mathrm{L}$ \\
\hline
\end{tabular}

c) Ligation reaction for pSHigh 


\section{Appendix 3: Restriction Enzyme Digestion}

Restriction enzyme reactions were carried out according to the manufacturer's instruction using appropriate buffers for each reaction. This information available from NEB. Checking for insertion of fragment to cloning vector required only a single enzyme digest desctibed in table A.3 a). Other digestion reactions involving the removal of fragments, or cutting of a plasmid before fragment insertion were carried out with a longer incubation period and appropriate enzymes. Restriction enzyme digestion reactions to remove the fragment from the cloning vector or pre ligation cutting of reporter plasmids are displayed in tables A.2 b) for pOT71 and c) for pSHigh. The reaction volume was altered in some cases to increase the final volume of cut DNA.

\section{A.3 Restriction Enzyme Digestion}

\begin{tabular}{lll}
\hline & Stock Concentration & In a $10 \mu \mathrm{L}$ reaction \\
\hline Cloning Vector + insert & Variable & $8 \mu \mathrm{L}$ \\
EcoRI & $1 \mathrm{U} / \mu \mathrm{L}$ & $1 \mu \mathrm{L}$ \\
EcoRI buffer & $10 \mathrm{x}$ & $1 \mu \mathrm{L}$ \\
\hline
\end{tabular}

a) Digestion of cloning vectors to check for insert

\begin{tabular}{lll}
\hline & Stock Concentration & In a $10 \mu \mathrm{L}$ reaction \\
\hline Cloning vector + insert/ pOT71 & variable & $7 \mu \mathrm{L}$ \\
EcoRI & $1 \mathrm{U} / \mu \mathrm{L}$ & $1 \mu \mathrm{L}$ \\
BamHI & $1 \mathrm{U} / \mu \mathrm{L}$ & $1 \mu \mathrm{L}$ \\
NEB buffer 2 & $10 \mathrm{x}$ & $1 \mu \mathrm{L}$ \\
\hline
\end{tabular}

b) Digestion of pOT71 plasmid before insertion of promoters 


\begin{tabular}{llc}
\hline Cloning vector + insert/ pSHigh & variable & $7 \mu \mathrm{L}$ \\
Kpnl & $1 \mathrm{U} / \mu \mathrm{L}$ & $1 \mu \mathrm{L}$ \\
BSA & $10 \mathrm{x}$ & $1 \mu \mathrm{L}$ \\
NEB buffer 3 & $10 x$ & $1 \mu \mathrm{L}$ \\
\hline
\end{tabular}

c) Digestion of pSHigh plasmid before insertion of promoters

\section{Appendix 4: Preparation of Calcium Competent DH5a Cells}

Calcium competent Escherichia coli DH5a cells were prepared for use in cloning procedures by the following protocol, ensuring all solutions and cells were kept on ice during preparation. TFB I and II are solutions used to induce competency as described by Hanahan et al [172] and are made up with the following concentrations of metal salts, glycerol and sterile distilled water before sterile filtration with a $0.22 \mu \mathrm{M}$ Millipore filter:

\begin{tabular}{|c|c|c|}
\hline TFB I: & for $100 \mathrm{~mL}$ & final concentration \\
\hline $\mathrm{MnCl}_{2}$ & $0.629 \mathrm{~g}$ & $50 \mathrm{mM}$ \\
\hline $\mathrm{CaCl}_{2}$ & 0.147 & $10 \mathrm{mM}$ \\
\hline K acetate & 0.294 & $30 \mathrm{mM}$ \\
\hline Glycerol & $15 \mathrm{~mL}$ & $15 \%$ \\
\hline SDW & $85 \mathrm{~mL}$ & \\
\hline TFB II & for $100 \mathrm{~mL}$ & final concentration \\
\hline Na MOPS (pH7) & 0.209 & $10 \mathrm{mM}$ \\
\hline $\mathrm{CaCl}_{2}$ & 0.629 & $75 \mathrm{mM}$ \\
\hline $\mathrm{KCl}$ & 0.075 & $10 \mathrm{mM}$ \\
\hline Glycerol & $15 \mathrm{~mL}$ & $15 \%$ \\
\hline
\end{tabular}


$\mathrm{DH} 5 a$ cells from a freezer stock were incubated with agitation at $37^{\circ} \mathrm{C}$ overnight in $3 \mathrm{ml}$ liquid cultures of $\mathrm{LB}$ with $10 \mathrm{mM} \mathrm{MgCl} 2$ with a negative media control to ensure there was no contamination. Cells were then inoculated into four $50 \mathrm{~mL}$ cultures of the LB with $10 \mathrm{mM} \mathrm{MgCl}_{2}$ in $250 \mathrm{~mL}$ flasks and grown for around two hours until their $\mathrm{OD}_{600}$ was between 0.4 and 1.0. Cells were then transferred to $50 \mathrm{~mL}$ flacon tubes and stored on ice to inhibit further growth. Cell suspensions were then spun down in a refrigerated centrifuge at $4{ }^{\circ} \mathrm{C}$ for 10 minutes at 5000rpm and resuspended in 40 $\mathrm{mLs}$ of competence inducing TFB I solution before further incubation on ice for two hours. Each culture was then spun down again at $4{ }^{\circ} \mathrm{C}$ for 10 minutes at $5000 \mathrm{rpm}$ and resuspended in 4mLs of TFB II solution and kept on ice. Cells suspended in TFB II were transferred to eppendorf tubes and snap frozen then stored at $-80^{\circ} \mathrm{C}$ until required for transformation. 


\section{Appendix 5: Preparation of Electrocompetent Mycobacterium smegmatis} $\mathrm{mc}^{2} 155$

Electrocompetent cells were prepared by a method revised from Sambrook and Russel Volume 1, 1.12-1.14 [157] .

Mycobacterium smegmatis $\mathrm{mc}^{2} 155$ cells from an LA plate were incubated with agitation at $37{ }^{\circ} \mathrm{C}$ overnight in $10 \mathrm{ml}$ liquid cultures of LB with a negative media control to ensure there was no contamination. $2-3 \mathrm{mLs}$ of this cells culture was inoculated into $100 \mathrm{mLs}$ of $\mathrm{LB}$ and grown for around five hours until their $\mathrm{OD}_{600}$ was between 0.5 and 1.0. Cells were then transferred to two $50 \mathrm{~mL}$ falcon tubes and stored on ice to inhibit further growth for 20 minutes. Cell suspensions were then spun down in a refrigerated centrifuge at $4{ }^{\circ} \mathrm{C}$ for 10 minutes at $5000 \mathrm{rpm}$ and re-

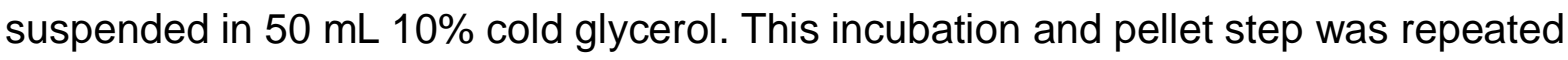
three times before cells were finally re-suspended in $2 \mathrm{mLs}$ of cold $10 \%$ glycerol before they were dispensed into eppendorf tubes and stored at $-80^{\circ} \mathrm{C}$ until required for transformation. 


\section{Appendix 6: Media used in this study for DH5a cells}

DH5a cells used for cloning were grown on solid media Luria Agar (LA) or Luria Broth (LB) both from Sigma. Overnight cultures of TA cloning vector containing cells were grown in LB supplemented with Ampicillin and Kanamycin when using pCR2.1 or just Ampicillin for pLUG vector. For blue/white colony selection, using $\beta$ galactosidase gene disruption, tranformed cells were grown on LA containg the same concentration of antibiotics as well as IPTG and Xgal.

Solid Media (LA)

- Kanamycin $50 \mu \mathrm{g} / \mathrm{mL}$

- Ampicillin $200 \mu \mathrm{g} / \mathrm{mL}$

- Xgal $40 \mu \mathrm{g} / \mathrm{mL}$

- IPTG $100 \mu \mathrm{g} / \mathrm{mL}$

Liquid Media (LB)

- Kanamycin $50 \mu \mathrm{g} / \mathrm{mL}$

- Ampicillin $200 \mu \mathrm{g} / \mathrm{mL}$ 


\section{Appendix 7: Media used in this study for $\mathrm{mc}^{2} 155$ cells}

All plasmids used in this study contained the Kanamycin resistance cassette ophA. Accordingly, all experiments using $\mathrm{mc}^{2} 155$ were supplemented with Kanamycin on both solid and liquied media. In order to obtain planktonic growth of liquid cultures, media was also supplemented with D-arabinose and $T_{w e e n}$. All media was autoclaved for sterilisation before use.

Solid Media (LA)

- Kanamycin $50 \mu \mathrm{g} / \mathrm{mL}$

Liquid Rich Media (LB)

- Kanamycin $50 \mu \mathrm{g} / \mathrm{mL}$

- D-arabinose $100 \mu \mathrm{g} / \mathrm{mL}$

- $0.1 \%$ Tween $_{80}$

Nutrient starvation experiments were carried out using Hartman deBonts minimal media made from a base of metal salts, Tween ${ }_{80}$ and SDW. Phosphate and Nitrate sources were added to this base from stock solutions names 100X P and N respectively, while glycerol was added as a Carbon source. 
Metal Salts

- $\mathrm{CaCl}_{2} \cdot 2 \mathrm{H}_{2} \mathrm{O}$

- $\mathrm{CoCl}_{2} \cdot 2 \mathrm{H}_{2} \mathrm{O}$

- $\mathrm{CuSO}_{4} \cdot 5 \mathrm{H}_{2} \mathrm{O}$

- EDTA

- $\mathrm{FeSO}_{4} \cdot 7 \mathrm{H}_{2} \mathrm{O}$

- $\mathrm{MgCl}_{2} \cdot 6 \mathrm{H}_{2} \mathrm{O}$

- $\mathrm{MnCl}_{2} \cdot 2 \mathrm{H}_{2} \mathrm{O}$

- $\mathrm{NaMoO}_{4} \cdot 2 \mathrm{H}_{2} \mathrm{O}$

- $\mathrm{ZnSO}_{4} \cdot 7 \mathrm{H}_{2} \mathrm{O}$

$100 \times$ Nitrogen (in 1L SDW) final concentration

$0.10 \mathrm{~g}$

$1 \mathrm{mM}$

$0.04 \mathrm{~g}$

$240 \mu \mathrm{M}$

$0.02 \mathrm{~g}$

$80 \mu \mathrm{M}$

$1.00 \mathrm{~g}$

$3.4 \mathrm{mM}$

$0.50 \mathrm{~g}$

$1.8 \mathrm{mM}$

$10.00 \mathrm{~g}$

$50 \mathrm{mM}$

$0.10 \mathrm{~g}$

$621 \mu \mathrm{M}$

$0.02 \mathrm{~g}$

$90 \mu \mathrm{M}$

$0.20 \mathrm{~g}$

$732 \mu \mathrm{M}$

- $\left(\mathrm{NH}_{4}\right)_{2} \mathrm{SO}_{4}$

$200 \mathrm{~g}$

$1.5 \mathrm{M}$

$100 \times$ Phosphates

- $\mathrm{K}_{2} \mathrm{HPO}_{4}$

$155 \mathrm{~g}$

$1 \mathrm{M}$

- $\mathrm{NaH}_{2} \mathrm{PO}_{4}$

$85 \mathrm{~g}$

$700 \mathrm{mM}$

HdeB base was made using $945 \mathrm{~mL}$ of SDW with $10 \mathrm{~mL}$ metal salts and 5 $\mathrm{mLs} 10 \%$ Tween $_{80}$. For each sort of media, mimimal, Carbon starvation, Nitrogen starvation and Phosphate starvation, $960 \mathrm{~mL}$ of $\mathrm{HdeB}$ base was used as well as nitrogen, carbon and phosphate sources as shown in the table below. For 
nutrient starvation experiments, the 4 levels of starvation were investigated using a range from $0 \%-100 \%$ as highlighted.

\begin{tabular}{|l|l|l|l|l|l|l|}
\hline & $\begin{array}{l}\text { HdeB } \\
\text { Base } \\
(\mathrm{mL})\end{array}$ & $\begin{array}{l}100 \mathrm{x} \\
\text { Nitrogen } \\
(\mathrm{mL})\end{array}$ & $\begin{array}{l}100 \mathrm{x} \\
\text { Phosphates } \\
(\mathrm{mL})\end{array}$ & $\begin{array}{l}10 \% \\
\text { Glycerol } \\
(\mathrm{mL})\end{array}$ & $\begin{array}{l}\text { SDW } \\
(\mathrm{mL})\end{array}$ & $\begin{array}{l}\text { 5M } \\
\text { NaMOPs } \\
(\mathrm{mL})\end{array}$ \\
\hline $\begin{array}{l}\text { Minimal } \\
\text { Media }\end{array}$ & 960 & 10 & 10 & 20 & 0 & \\
\hline $\begin{array}{l}\text { Nitrogen } \\
\text { Starvation }\end{array}$ & 960 & $0.1-10$ & 10 & 20 & $9.9-0.1$ & \\
\hline $\begin{array}{l}\text { Phosphate } \\
\text { starvation }\end{array}$ & 960 & 10 & $1-10$ & 20 & $10-1$ \\
\hline $\begin{array}{l}\text { Carbon } \\
\text { Starvation }\end{array}$ & 960 & 10 & 10 & $8-20$ & $12-0$ & \\
\hline
\end{tabular}

Table A.3 Alterations to HdeB media for nutrient starvation 